\title{
Eye Movements in Reading and Information Processing: 20 Years of Research
}

\author{
Keith Rayner \\ University of Massachusetts at Amherst
}

\begin{abstract}
Recent studies of eye movements in reading and other information processing tasks, such as music reading, typing, visual search, and scene perception, are reviewed. The major emphasis of the review is on reading as a specific example of cognitive processing. Basic topics discussed with respect to reading are (a) the characteristics of eye movements, (b) the perceptual span, (c) integration of information across saccades, (d) eye movement control, and (e) individual differences (including dyslexia). Similar topics are discussed with respect to the other tasks examined. The basic theme of the review is that eye movement data reflect moment-to-moment cognitive processes in the various tasks examined. Theoretical and practical considerations concerning the use of eye movement data are also discussed.
\end{abstract}

Many studies using eye movements to investigate cognitive processes have appeared over the past 20 years. In an earlier review, I (Rayner, 1978b) argued that since the mid-1970s we have been in a third era of eye movement research and that the success of research in the current era would depend on the ingenuity of researchers in designing interesting and informative studies. It would appear from the vast number of studies using eye movement data over the past 20 years that research in this third era is fulfilling the promise inherent in using eye movement behavior to infer cognitive processes. The first era of eye movement research extended from Javal's initial observations concerning the role of eye movements in reading in 1879 (see Huey, 1908) up until about 1920. During this era, many basic facts about eye movements were discovered. Issues such as saccadic suppression (the fact that we do not perceive information during an eye movement), saccade latency (the time that it takes to initiate an eye movement), and the size of the perceptual span (the region of effective vision) were of concern in this era. The second era, which coincided with the behaviorist movement in experimental psychology, tended to have a more applied focus, and little research was undertaken with eye movements to infer cognitive processes. Although classic work by Tinker (1946) on reading and by Buswell (1935) on scene perception was carried out during this era, in retrospect, most of the work seems to have focused on the eye movements per se (or on surface aspects of the task being investigated). Tinker's (1958) final review ended on the rather pessimistic note that almost every-

Preparation of this article was supported by a Research Scientist Award from the National Institute of Mental Health (MH01255) and by Grants HD 17246 and HD 26765 from the National Institutes of Health. Thanks are extended to Ken Ciuffreda, Charles Clifton, David Irwin, and Alexander Pollatsek for their helpful comments on prior versions of this article.

Correspondence concerning this article should be addressed to Keith Rayner, Department of Psychology, University of Massachusetts, Amherst, Massachusetts 01003 . Electronic mail may be sent to rayner@ psych.umass.edu. thing that could be learned about reading from eye movements (given the technology at the time) had been discovered. Perhaps that opinion was widely held, because between the late $1950 \mathrm{~s}$ and the mid-1970s little research with eye movements was undertaken.

The third era of eye movement research began in the mid1970s and has been marked by improvements in eye movement recording systems that have allowed measurements to be more accurate and more easily obtained. It is beyond the scope of the present review to detail all of the technological advancements that have been made. Numerous works have dealt with methods of analyzing eye movement data (see Kliegl \& Olson, 1981; Pillalamarri, Barnette, Birkmire, \& Karsh, 1993; Scinto \& Barnette, 1986), and much has been learned about the characteristics of various eye-tracking systems (see Deubel \& Bridgeman, 1995a, 1995b; Muller, Cavegn, d'Ydewalle, \& Groner, 1993). More important, the era has yielded tremendous technological advances that have made it possible to interface laboratory computers with eye-tracking systems so that large amounts of data can be collected and analyzed. These technological advances have also allowed for innovative techniques to be developed in which the visual display is changed contingent on the eye position. In the eye-contingent display change paradigm (McConkie, 1997; McConkie \& Rayner, 1975; Rayner, 1975b; Reder, 1973), eye movements are monitored, and changes are made in the visual display that the reader is looking at, contingent on when the eyes move (or at some other critical point in the fixation). Finally, the development of general theories of language processing has made it possible to use eye movement records for a critical examination of the cognitive processes underlying reading.

In the present article, recent studies of eye movements in reading and other information processing tasks are examined. Since the last review in this journal (Rayner, 1978b), there have been many reviews of eye movement research (Kennedy, 1987; Lévy-Schoen \& O'Regan, 1979; O'Regan, 1990; Pollatsek, 1993; Rayner, 1984, 1993, 1995, 1997; Rayner \& Pollatsek, 1987, 1992; G. Underwood, 1985). However, none of them are 
comprehensive: Some deal only with reading (or scene perception), and most of them advocate a particular point of view. The goal of the present article is to provide a comprehensive review of eye movement research over the past 20 years. Since 1978, many eye movement studies have appeared in edited books. Because the quality of the research reported in such books is somewhat variable, the focus of the current review is on studies that have appeared in peer-reviewed journals.

\section{Basic Characteristics of Eye Movements in Information Processing}

When we read, look at a scene, or search for an object, we continually make eye movements called saccades. Between the saccades, our eyes remain relatively still during fixations for about $200-300 \mathrm{~ms}$. There are differences in these two measures as a function of the particular task (see Table 1). Saccades are rapid movements of the eyes with velocities as high as $500^{\circ}$ per second. Sensitivity to visual input is reduced during eye movements; this phenomenon is called saccadic suppression (Matin, 1974) and has been the topic of considerable debate. We do not obtain new information during a saccade, because the eyes are moving so quickly across the stable visual stimulus that only a blur would be perceived (Uttal \& Smith, 1968). More important, however, masking caused by the information available before and after the saccade makes it such that we do not perceive any type of blurring effect (Brooks, Impelman, \& Lum, 1981; Campbell \& Wurtz, 1979; Chekaluk \& Llewellyn, 1990). However, some suppression is found even when masking is eliminated (Riggs, Merton, \& Morton, 1974), which suggests that there is a central inhibitory contribution to saccadic suppression as well.

The velocity of the saccade is a monotonic function of how far the eyes move; it rapidly rises during the saccade to a maximum that occurs slightly before the midpoint of the movement and then drops at a slightly slower rate until the target location is reached. The duration of a saccade is also influenced by the distance covered; a $2^{\circ}$ saccade typical of reading takes around $30 \mathrm{~ms}$, whereas a $5^{\circ}$ saccade, typical of scene perception, takes around 40-50 ms (R. A. Abrams, Meyer, \& Kornblum, 1989; Rayner, 1978a).

An issue that has received considerable attention recently is

Table 1

Approximate Mean Fixation Duration and Saccade Length in Reading, Visual Search, Scene Perception, Music Reading, and Typing

\begin{tabular}{lcl}
\hline \multicolumn{1}{c}{ Task } & $\begin{array}{c}\text { Mean fixation } \\
\text { duration (ms) }\end{array}$ & $\begin{array}{c}\text { Mean saccade size } \\
\text { (degrees) }\end{array}$ \\
\hline Silent reading & 225 & 2 (about 8 letters) \\
Oral reading & 275 & 1.5 (about 6 letters) \\
Visual search & 275 & 3 \\
Scene perception & 330 & 4 \\
Music reading & 375 & 1 \\
Typing & 400 & 1 (about 4 letters) \\
\hline
\end{tabular}

Note. Values are taken from a number of sources and vary depending on a number of factors (see Rayner, 1984). whether or not cognitive processing activities are suspended during a saccade (Boer \& van der Weijgert, 1988; W. Hansen \& Sanders, 1988; Irwin \& Carlson-Radvansky, 1996; Irwin, Carlson-Radvansky, \& Andrews, 1995; Matin, Shao, \& Boff, 1993; Sanders \& Houtmans, 1985; Sanders \& Rath, 1991; Van Duren, 1993; Van Duren \& Sanders, 1992, 1995). Some of these studies have found evidence to suggest that some cognitive activities are suppressed during saccades. Because the tasks used in most of these studies are relatively simple, it will be interesting to determine whether thinking is suspended during saccades in more complex tasks, such as reading and scene perception. Certainly, people are not aware of pauses in mental activity during eye movements, but because saccade durations are so brief, any disruptions might not be particularly salient. Recently, Irwin (1998) reported some studies demonstrating that lexical processing is not suppressed during saccades.

Saccades need to be distinguished from three other types of eye movements: pursuit, vergence, and vestibular eye movements. Pursuit eye movements occur when our eyes follow a moving target; the velocity of pursuit eye movements is markedly slower than saccades and, if the target is moving quickly across our visual field, we often make saccades to catch up with the target (White, 1976). Vergence eye movements occur when we move our eyes inward, toward each other, in order to fixate on a nearby object. Vestibular eye movements occur when the eyes rotate to compensate for head and body movements in order to maintain the same direction of vision. Although pursuit, vergence, and vestibular eye movements are important and extensively studied (see Ciuffreda \& Tannen, 1995, and Leigh \& Zee, 1991, for more details), saccadic eye movements are more relevant in typical information processing tasks. ${ }^{1}$

Finally, three types of small movements of the eyes need to be mentioned: nystagmus, drifts, and microsaccades. Although researchers interested in eye movements in information processing tasks typically discuss fixations as the period of time when the eyes are still, the term fixation is something of a misnomer. The eyes are never really still, because there is a constant tremor of the eyes called nystagmus. Such tremors of the eyes are quite small, and their exact nature is somewhat unclear, though it is often assumed that the movements are related to perceptual activity and help the nerve cells in the retina to keep firing. Drifts and microsaccades tend to be somewhat

\footnotetext{
' From a number of studies, it is known that the coordination of horizontal and vertical saccades is very accurate in adults when the stimulus is a visually presented target (Bains, Crawford, Cadera, \& Vilis, 1992; Collewijn, Erkelens, \& Steinman, 1988a, 1988b). It is typically assumed that the eyes move conjugately during tasks like reading. However, Collewijn et al. ( 1988a) found that the movements of the abducting (temporally moving) eye are somewhat larger than the corresponding movements of the adducting (nasally moving) eye in simple scanning tasks. Similar observations were reported by Heller and Radach (1995) for reading and other complex tasks. Bassou, Pugh, Granie, and Morucci (1993) and Ygge and Jacobson (1994) reported that although the eyes of fifth-grade readers tended to be well-coordinated spatially, they were not always closely conjugated spatially. Henriks (1996) recently examined vergence movements of skilled readers and found that the eyes converge during reading. As she noted, this finding is in contrast to earlier reports (E. A. Taylor, 1966) in which the eyes were found to diverge during reading.
} 
larger movements than the nystagmus movements. Although the reasons for these movements are not completely clear, it appears that the eyes occasionally drift (i.e., make small, slow movements) because of less-than-perfect control of the oculomotor system by the nervous system. When this happens, there is often a small microsaccade (a much more rapid movement) to bring the eyes back to where they were. Most experimenters interested in reading assume that these small movements are "noise"' and adopt scoring procedures that ignore them. For example, most researchers lump together successive fixations that are on adjacent characters as a single fixation. Another alternative is a more sophisticated procedure in which fixations are pooled if the two fixations are on adjacent characters and one is short (i.e., less than $100 \mathrm{~ms}$ ).

\section{Saccade Latency}

There is a latency period associated with making a saccade, because they are motor movements that require time to plan and execute. Even if uncertainty about when or where to move the eyes is eliminated, saccade latency is at least $150-175 \mathrm{~ms}$ (R. A. Abrams \& Jonides, 1988; Rayner, Slowiaczek, Clifton, \& Bertera, 1983; Salthouse \& Ellis, 1980; Salthouse, Ellis, Diener, \& Somberg, 1981), which suggests that saccade programming is done in parallel with comprehension processes in reading.

Studies dealing with saccade latency are legion, and a complete review is beyond the scope of the present article. However, some important facts have been learned about saccade latency that may be relevant to understanding eye movement behavior in information processing tasks (Becker \& Jürgens, 1979; Crawford; 1996; Findlay, 1992; Findlay \& Walker, in press; Heywood \& Churcher, $1980)$. First, there are separate decision processes involved in computing when and where to move the eyes (Aslin \& Shea, 1987; Becker \& Jürgens, 1979). Second, although saccades in simple reaction-time experiments are often characterized as reflexive, there is also evidence that cognitive processes can influence the latency (Deubel, 1995). For example, in the antisaccade paradigm (Hallett, 1978), saccades are voluntarily directed away from a peripheral target, and latencies increase. Likewise, when a number of saccades are planned in a sequence, latencies of the initial saccade increase (Crawford, 1990; Inhoff, 1986; Zingale \& Kowler, 1987); as eccentricity of the target increases, latencies can increase (Kalesnykas \& Hallett, 1995). Third, increasing the saccade latency generally leads to increased accuracy in locating a target (Jacobs, 1987a; Nazir \& Jacobs, 1991). Fourth, when saccades are made to targets consisting of two elements, in reasonably close proximity, the first saccade goes to some intermediate location. This is referred to as the global or center of gravity effect (Deubel, Wolf, \& Hauske, 1984; Findlay, 1982; Ottes, Van Ginsbergen, \& Eggermont, 1984). If one element is larger or more intense (or brighter), then the saccade tends to land closer to that target in comparison to a condition in which the two elements are identical. Instructions to be more careful influence where the eyes land (Coëffé \& O'Regan, 1987; Kowler \& Blaser, 1995) but also increase saccade latency. Fifth, when a fixation point disappears prior to the appearance of a target, latency decreases (M. E. Cohen \& Ross, 1977; Kingstone \& Klein, 1993a; L. E. Ross \& Ross, 1980; S. M. Ross \& Ross, 1981). This speeding up (called the gap effect) relates to the temporal warning provided by the fixation-point offset; visual offset is more effective than a neutral (auditory) cue, which in turn is more effective than onset of visual stimulation at the fixation point (Findlay, 1992). This pattern of results suggests that some process that may be termed relinquishing of attention is involved in generating a saccade. Interest in these results has intensified recently because of the suggestion that there is a separate category of saccades, called express saccades, with very short latencies (Cavegn \& d'Ydewalle, 1996; B. Fischer, 1992; B. Fischer \& Boch, 1983; B. Fischer \& Rampsperger, 1984; B. Fischer \& Weber, 1993). At the moment, there is some debate about (a) whether or not there is a bimodal distribution of saccade latencies (with express saccades representing one peak of the distribution and normal saccades representing the other) and (b) the phenomenon in general (Findlay, 1992; Kingstone \& Klein, 1993b; A. B. Sereno, 1992). Even if there were no controversy surrounding express saccades per se, there are questions about the functional utility of such shortlatency saccades for normal information processing tasks (M. H. Fischer \& Rayner, 1993): Inhoff, Topolski, Vitu, and O'Regan (1993) found no evidence for express saccades or a bimodal distribution of fixation durations during reading.

\section{The Visual Field and Acuity}

We make saccades so frequently because of acuity limitations. As we look straight ahead, the visual field can be divided into three regions: foveal, parafoveal, and peripheral. Although acuity is very good in the fovea (the central $2^{\circ}$ of vision), it is not nearly so good in the parafovea (which extends out to $5^{\circ}$ on either side of fixation), and it is even poorer in the periphery (the region beyond the parafovea). Hence, we move our eyes so as to place the fovea on that part of the stimulus we want to see clearly. Of course, characteristics of the stimulus in parafoveal or peripheral vision influence whether or not a saccade needs to be made to identify it. For example, if a word of normalsize print is presented in parafoveal vision, it is identified more quickly and accurately when a saccade is made (Jacobs, 1986, 1987a; Rayner \& Morrison, 1981). However, if an object or large letter is presented as the stimulus, it can often be identified in peripheral vision without a saccade (Pollatsek, Rayner, \& Collins, 1984). Sanders (1993) showed that the visual field can be divided into regions where (a) a stimulus can be identified without an eye movement, (b) it is necessary to make an eye movement to identify the stimulus, and (c) it is necessary to make a head movement to identify the stimulus.

\section{Eye Movements and Attention}

Although it is often necessary to move our eyes to identify objects in our environment, we can move attention without moving our eyes (Posner, 1980). The relationship between attention and eye movements has been extensively investigated (M. H. Fischer, in press; Klein, 1980; Klein, Kingstone, \& Pontefract, 1992; Rafal, Calabresi, Brennan, \& Sciolto, 1989; Remington, 1980; Reuter-Lorenz \& Fendrich, 1992; Shepherd, Findlay, \& Hockey, 1986) but is beyond the scope of the present review. However, with complex stimuli, it is more efficient to move our eyes than to move attention (He \& Kowler, 1992; Sclingensie- 
pen, Campbell, Legge, \& Walker, 1986). Furthermore, there is evidence suggesting that attention precedes a saccade to a given location in space (Hoffman \& Subramaniam, 1995; Kowler, Anderson, Dosher, \& Blaser, 1995; Rayner, McConkie, \& Ehrlich, 1978; Remington, 1980) and that attentional movements and saccades are obligatorily coupled (Deubel \& Schneider, 1996; but see Stelmach, Campsall, \& Herdman, 1997, for conflicting data). Although we can easily decouple the locus of attention and eye location in simple discrimination tasks (Posner, 1980), in complex information processing tasks such as reading, the link between the two is probably quite tight.

\section{Developmental Changes in Eye Movements}

The characteristics of children's eye movements differ somewhat from those of adults. Preschool children exhibit more frequent small saccades and drifts during maintained fixation; saccadic latency is usually longer, and saccade accuracy is usually less precise in preschool children than in adults when scanning a scene (Kowler \& Martins, 1985). However, the shapes of the frequency distributions of fixation durations for children, adults, and even infants are quite similar (Hainline, Turkel, Abramov, Lemerise, \& Harris, 1984; Harris, Hainline, Abramov, Lemerise, \& Camenzuli, 1988). Although frequency distributions of fixation durations of elderly adults look like those of younger adults, saccade latency increases with age (Abel, Troost, \& Dell'Osso, 1983; Pirozzolo \& Hansch, 1981).

\section{Measuring Eye Movements}

Eye movements are monitored in many different ways. Eyetracking systems are currently in use that rely on (a) surface electrodes (which are fairly good at measuring saccade latency but not good at measuring location), (b) infrared corneal reflections, (c) video-based pupil monitoring, (d) infrared Purkinje image tracking, and (e) search coils attached like contact lenses to the surface of the eyes. Although there has been some discussion concerning the measurement, evaluation, and reporting of eye movement data (Harris, Abramov, \& Hainline, 1984; Heller, 1983; Inhoff \& Radach, 1998; McConkie, 1981; McConkie, Wolverton, \& Zola, 1984; Nodine, Kundel, Toto, \& Krupinski, 1992), no measurement standards have been adopted, and many methodological issues remain unaddressed or unresolved ( see Inhoff \& Radach, 1998, for a good discussion of these issues ). Despite this fact, most of the important findings discussed in this review have been replicated across different labs.

\section{Eye Movements in Reading}

Eye movements differ somewhat for reading silently versus aloud (see Table 1). When reading aloud, or when reading silently while listening to a voice reading the same text, mean fixation durations are longer than in silent reading, and the eyes. tend to get ahead of the voice; consequently, there are many fixations in which the eyes appear to be holding in place so as to not get too far ahead of the voice (Lévy-Schoen, 1981). The vast majority of the research on reading reviewed here is for silent reading.
When reading English, eye fixations last about $200-250 \mathrm{~ms}$ and the mean saccade size is 7-9 letter spaces (see Table 1). Letter spaces are the appropriate metric to use, because the number of letters traversed by saccades is relatively invariant when the same text is read at different distances, even though the letter spaces subtend different visual angles (Morrison, 1983; Morrison \& Rayner, 1981; O'Regan, 1983; O'Regan, LévySchoen, \& Jacobs, 1983). ${ }^{2}$ The primary function of a saccade is to bring a new region of text into foveal vision for detailed analysis, because reading on the basis of only parafoveal or peripheral information is difficult to impossible (Rayner \& Bertera, 1979; Rayner, Inhoff, Morrison, Slowiaczek, \& Bertera, 1981). Whereas a majority of the words in a text are fixated during reading, many words are skipped so that foveal processing of each word is not necessary. For example, content words are fixated about $85 \%$ of the time, whereas function words are fixated about $35 \%$ of the time (Carpenter \& Just, 1983; Rayner \& Duffy, 1988). Function words are fixated less frequently than content words, because they tend to be short, and there is a clear relationship between the probability of fixating a word and its length: As length increases, the probability of fixating a word increases (Rayner \& McConkie, 1976); 2-3 letter words are only fixated around $25 \%$ of the time, whereas words 8 letters or longer are almost always fixated (and often fixated more than once).

Although most saccades in reading English are made from left to right, readers do not relentlessly go forward: About 10$15 \%$ of the saccades are regressions (right-to-left movements along the line or movements back to previously read lines). Many regressions tend to be only a few letters long and could be due to the reader making too long of a saccade, in which case a short saccade to the left may be necessary for reading to proceed efficiently. Short within-word regressive saccades may also be due to problems that the reader has processing the currently fixated word. Longer regressions (more than 10 letter spaces back along the line or to another line) occur because the reader did not understand the text. In such cases, good readers are very accurate in sending their eyes to that part of text that caused them difficulty (Frazier \& Rayner, 1982; Kennedy, 1983; Kennedy \& Murray, 1987a, 1987b; Murray \& Kennedy, 1988), whereas poor readers engage in more backtracking through the text (Murray \& Kennedy, 1988).

On return sweeps from the end of one line to the beginning of the next, readers often undershoot and make small corrective movements to the left. Because corrective saccades are often made following return sweeps, it should not be assumed that readers place their fixation to correspond to the beginning of a line. Rather, the first and last fixations on a line are generally $5-7$ letter spaces from the ends of a line. Thus, about $80 \%$ of the text typically falls between the extreme fixations. The first fixation on a line tends to be longer than other fixations (Heller, 1982; Rayner, 1977), and the last is shorter (Rayner, 1978b).

\footnotetext{
${ }^{2}$ However, it should be noted that fixation time increases somewhat when the visual angle subtended by a letter is appreciably smaller than in normal reading. This is because the letters are more difficult to discriminate when the text is further away from the eyes. Thus, letter spaces are the appropriate metric of saccade size for relatively normal-sized print; if the text is too small or too large, the principle would not hold.
} 
Furthermore, readers tend to not fixate in the blank spaces between sentences (S. G. Abrams \& Zuber, 1972; Rayner, 1975a).

Although average values for fixation duration, saccade length, and frequency of regression are cited above, there is considerable between-reader variability for all three measures. More important, there is variability for a given reader within a single passage of text so that fixation durations range from under 100 $\mathrm{ms}$ to over $500 \mathrm{~ms}$, and saccades vary from 1 to over 15 letter spaces (see Figure 1). Actually, fixations as short as $50 \mathrm{~ms}$ sometimes appear in the eye movement record during reading. Saccades as long as 15 letter spaces are quite rare and often occur immediately following a regression in which readers typically make a long saccade to place the eyes ahead of where they were prior to making the regression.

Eye movements are also influenced by textual and typographical variables. For example, as text becomes conceptually more difficult, fixation duration increases, saccade length decreases, and the frequency of regressions increases (Jacobson \& Dod-
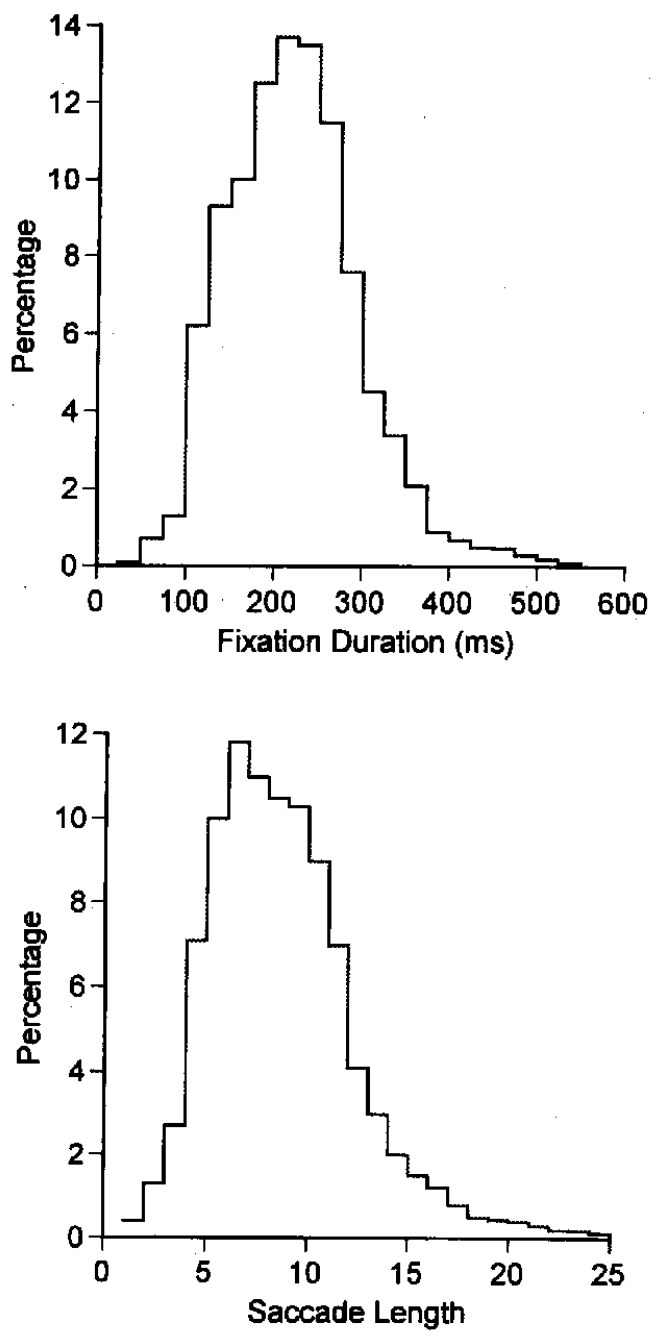

Figure 1. Frequency distributions for fixation durations and forward saccade lengths. Fixation durations are in milliseconds, and saccade length is in character spaces. well, 1979; Rayner \& Pollatsek, 1989). If the text looks fairly normal, typographical variables tend to have a relatively minor influence. However, factors such as the quality of the print (variations in fonts), line length, and letter spacing (Kolers, Duchnicky, \& Ferguson, 1981; Morrison \& Inhoff, 1981) influence eye movements. Characteristics of the writing system also influence eye movements (Osaka, 1989; Peng, Orchard, \& Stern, 1983; Sun, Morita, \& Stark, 1985).

A crucial point that has emerged recently is that eye movement measures can be used to infer moment-to-moment cognitive processes in reading (Just \& Carpenter, 1980; McConkie, Hogaboam, Wolverton, Zola, \& Lucas, 1979; Rayner, 1978b; Rayner, Sereno, Morris, Schmauder, \& Clifton, 1989) and that the variability in the measures reflects on-line processing. For example, there is now abunidant evidence that the frequency of a fixated word influences how long readers look at the word (Inhoff \& Rayner, 1986; Rayner \& Duffy, 1986). Thus, properties of the fixated word modulate the fixation time and result in variability in fixation times. There is, of course, a purely motoric component to this variability (Kowler \& Anton, 1987), because when spatial and temporal uncertainty about where and when to move the eyes is eliminated, there is still variability in the latency of eye movements (Rayner, Slowiaczek, et al., 1983; Salthouse \& Ellis, 1980). Similarly, there is variability in where the eyes land on a target (Coëffé \& O'Regan, 1987; Findlay, 1982 ). Although this noise of motoric variability makes it difficult to interpret the cognitive signal in the eye movement record, it is clear that the signal is there, and great strides have been made over the past 20 years in understanding the relationship between reading and eye movements. One simple point consistent with this claim is that, in reading, there is no correlation between fixation duration and saccade length (Rayner \& McConkie, 1976), whereas in nonreading situations, in which the necessity of linguistic processing is eliminated, there is a correlation: the longer the saccade, the longer the next fixation (Kapoula, 1983; Nattkemper \& Prinz, 1986). The difference between these two situations suggests that the difficulty of online language processing associated with reading wipes out any simple correlation. However, it is important to note that although there is no correlation between fixation duration and saccade length across extended text, correlations can be obtained locally between a given fixation duration and saccade length (Pollatsek, Rayner, \& Balota, 1986), indicating that processing difficulty influences both eye movement variables.

\section{What Is the Best Measure of Processing Time?}

A major issue concerns how to best summarize the eye movement record to understand cognitive processing. This issue is particularly relevant to the question of how to measure the temporal processing associated with a given region of text. If the unit of analysis is larger than a word, then the total first-pass fixation time on that unit is generally used as the primary measure of interest. It is important, when analyzing such regions, to distinguish between first-pass (i.e., the initial reading consisting of all forward fixations ) and second-pass (i.e., rereading) reading time for the region. There is some controversy about how best to analyze a region when readers make regressions ( see Altmann, 1994; Rayner \& Sereno, 1994b, 1994c). For example, 
Rayner and Sereno (1994c) noted that, when readers enter a region and then quickly make a regression out of that region, the first-pass time is very short in comparison to when the reader does not regress. How best to analyze such regressions is uncertain. Some researchers simply record first-pass reading time and then examine the pattern of regressions out of the target region. Others have argued for regression-path durations analyses (Konieczny, Hemforth, Scheepers, \& Strube, 1997) or cumulative region reading time analyses (Brysbaert \& Mitchell, 1996). With these analyses, reading time represents the sum of all fixations starting with the first fixation in a region and ending with the first forward saccade past the region under consideration $^{3}$ (see Liversedge, Paterson, \& Pickering, 1998, for further discussion of these issues).

Issues have also arisen concerning the fact that comparisons often are made between regions of text that contain different words, with some regions being longer than others. One procedure is to divide the reading time (first pass or otherwise) by the number of letters in the region to yield a millisecondper-character reading time. However, this procedure has been demonstrated to yield a nonlinear function of word length on reading time, especially in short regions (Trueswell, Tanenhaus, \& Garnsey, 1994). Thus, when the length of a region differs, a more appropriate procedure is to analyze the deviations from expected reading times as determined by the best linear fit for the reading time as a function of the number of letters in the region (Ferreira \& Clifton, 1986; Trueswell et al., 1994).

When a word is the unit of analysis, then the appropriate measure to use is also controversial (Inhoff \& Radach, 1998). If readers always were to make one and only one fixation on a word, then there would be little problem: The fixation duration on the word would be the measure of processing time. However, words are sometimes fixated more than once, and sometimes they are skipped. The problem of multiple fixations has led to different solutions. Using the mean fixation duration on a word is clearly inadequate, because it underestimates the time the eyes are actually on the word (i.e., a $200-\mathrm{ms}$ fixation and a $150-\mathrm{ms}$ fixation on the same word would yield a mean fixation duration on the word of $175 \mathrm{~ms}$ ). Likewise, the strategy of including only words that are fixated once (single fixation duration) is problematic, because some words are fixated more than once, and some are skipped altogether; such a strategy may result in the elimination of too much data. Thus, the two most frequently used measures are the first fixation duration and the gaze duration on a word. Gaze duration represents the sum of all fixations made on a word prior to a saccade to another word. First fixation duration is the duration of the first fixation on a word regardless of whether it is the only fixation on a word or the first of multiple fixations on a word.

The argument over which measure is best to use as an index of processing time partly depends on what is being examined. Inhoff (1984) argued that first fixation duration and gaze duration measure different processes. In his data, first fixation duration and gaze duration both were affected by word frequency, but only gaze duration was affected by the predictability of the word in the context. He thus posited that first fixation is a measure of lexical access, whereas gaze duration reflects text integration processes as well. However, it now appears that this distinction does not hold up in general and that for much of the time, although not always, first fixation duration and gaze duration yield similar results. Rayner and Pollatsek (1987) argued that the data suggest that if a cognitive operation is very fast it affects first fixation duration, and if it is a bit slower it may affect gaze duration. Assuming that the gaze versus first fixation duration problem were solved, there is still the problem of trying to assess the average time spent processing a word. The problem is that words are processed when they are not fixated. Fisher and Shebilske (1985) showed this by having readers read text as their eye movements were monitored. They then had another group read the same text after deleting the words that the first group skipped over (function words and other short words ). The second group had a difficult time understanding the text.

Just and Carpenter (1980) assigned a value of $0 \mathrm{~ms}$ to words that were not fixated in their gaze duration analysis. However, this procedure really does not solve the problem, because words that are not fixated are clearly processed by the reader (generally on the fixation prior to the skip). Subsequently, as a means of dealing with word skipping, Carpenter and Just (1983) proposed a conditionalized gaze duration, which is the mean gaze duration, given that the word has been fixated for at least $50 \mathrm{~ms}$. The problem with this measure is that it ignores the time spent in a fixation processing words to the right of the fixated word. As discussed in detail later in this review, when the word to the right of fixation is not identified, some initial parafoveal processing occurs. Rayner and Pollatsek (1987) suggested that conditionalized gaze duration would be better if conditionalized on fixating the word for $50 \mathrm{~ms}$ and not skipping the next word. A second solution to the problem is the read to the right of gaze (RRG) algorithm (Blanchard, 1985; Hogaboam \& McConkie, 1981). The RRG measure sums fixations on words receiving more than one fixation; when words are skipped, the fixation time is equally distributed between the last word fixated and the word that was skipped. Both the conditionalized gaze and the RRG measures assume that (except for skipping) a word is processed only when it is fixated. However, as just noted, parafoveal information is extracted from a word on most fixations and facilitates identification on the subsequent fixation. Rayner and Pollatsek (1987) suggested that perhaps this preview benefit should be added to the fixation time on the word and should be subtracted from the time spent on the prior word. All of this assumes that these processes can be precisely identified and that words are processed in series. If some processes overlap, then the calculation of processing times would be even more complex. In addition, there is the problem that the processing of a word is not always completed by the time the eyes move, as there are spillover effects: Time processing a word can "spill over" onto the next word (Rayner \& Duffy, 1986; Rayner, Sereno, et al., 1989). To the extent that the processing of a word spills over onto subsequent fixations (McConkie, Zola, \& Blanchard, 1984; Rayner, Sereno, et al., 1989), these measures are in error.

It thus appears that any single measure of processing time per word is a pale reflection of the reality of cognitive processing.

\footnotetext{
${ }^{3}$ Rayner and Duffy (1986) and Duffy, Morris, and Rayner (1988) used a virtually identical procedure to establish the time needed to understand the information in a region.
} 
Therefore, the strategy of analyzing large amounts of text with a single measure of processing is likely to be of limited value in measuring on-line processing. Just and Carpenter (1980) adopted such a strategy when they used multiple regression techniques to analyze eye movements over large amounts of text. Their procedure has been criticized by a number of researchers (Fisher \& Shebilske, 1985; Hogaboam \& McConkie, 1981; Kliegl, Olson, \& Davidson, 1982; Slowiaczek, 1983). Those criticisms are not repeated here, but many of them follow because any such procedure must make unjustifiable simplifying assumptions about the relationship between the measure being used (in this case, gaze duration) and cognitive processing during reading. An alternative strategy that many researchers have adopted is to select target locations in texts for careful analysis (usually on the basis of theoretical considerations) and examine many different measures (such as first fixation duration, single fixation duration, gaze duration, probability of fixating a target word, number of fixations on the target word, saccade length to and from the target word, and spillover effects). By doing so, it is possible to draw some reasonable inferences about reading processes (Rayner, Sereno, et al., 1989; Schmauder, 1992).

\section{Saccade Durations and Gaze Duration}

A recent controversial issue concerns whether or not saccade durations should be included in the computation of gaze duration. Most researchers have followed the lead of Just and Carpenter (1980) and have used only fixation duration values in computing gaze durations. However, Irwin (1998) recently argued that lexical processing continues during saccades and that saccade duration should be added into the gaze computation (see also Inhoff \& Radach, 1998). This is a reasonable suggestion. On the other hand, because intraword saccade durations are quite brief and because the frequency of refixating a word before moving to another word is relatively low, any effect of adding saccade duration into the gaze duration measure is quite minimal. For example, as noted previously, readers' gaze durations are longer on low-frequency words than on high-frequency words. When saccade duration is added into the gaze measure, the size of the effect (which is typically around $50 \mathrm{~ms}$ ) increases by roughly 3-8 ms. In addition, if saccade durations are to be added to gaze durations, it is unclear what should be done with saccade durations for interword saccades. Should they be added to the word just fixated or the word that is the target of the next saccade? Thus, there are unresolved issues regarding what to do with saccade durations. For gaze durations on single target words, researchers would be wise to determine if adding saccade duration has an effect in a given study. At this point, it appears that it typically does not. On the other hand, when regions larger than a single word represent the unit of analysis, effects of saccade duration are larger and hence are more likely to aid in revealing differences between conditions.

\section{Eye Movements and Perceptual Processes in Reading}

\section{The Acquisition of Information During Reading}

With respect to the sequence of saccades and fixations, when is information acquired from the text during reading? Wolverton and Zola (1983) used the eye-contingent display change technique to replace an entire line of text with (a) another line of text, (b) random letters, or (c) a row of $X \mathrm{~s}$ for a $20-\mathrm{ms}$ period during either a saccade or a fixation. After the $20-\mathrm{ms}$ period, the normal text reappeared. If the text was altered at any point during the fixation (including the first $20 \mathrm{~ms}$ of the fixation), the change was noticed and disrupted reading. However, if the change took place during the saccade, it was not noticed and did not disrupt reading (see also Ishida \& Ikeda, 1989). Thus, new information is acquired from the text only during fixations.

Rayner et al. (1981; see Ishida \& Ikeda, 1989; Slowiaczek \& Rayner, 1987) presented a masking pattern at various points in a fixation and found that reading proceeded quite smoothly if the text was presented for $50 \mathrm{~ms}$ or more prior to the onset of the mask. ${ }^{4}$ On the other hand, Blanchard, McConkie, Zola, and Wolverton (1984) argued that visual information may be acquired within the fixated region at any point during a fixation as needed by comprehension processes. They replaced a given target word ( such as tombs) in a sentence with another word (such as bombs) at various points during a fixation and had readers make forcedchoice decisions about whether or not they had seen these words (as well as foils) during reading. Their basic finding was that the longer the word was present in the text, the higher the probability of indicating that it was present. However, words presented for only $50 \mathrm{~ms}$ (at either the beginning or end of a fixation) were sometimes responded to positively. Blanchard et al. argued that visual information is acquired whenever needed during a fixation. Actually, the results of these two sets of experiments are really not so contradictory; both of them emphasize the flexibility that readers have in dealing with situations in which masks suddenly appear or words suddenly change during a fixation. For the sake of parsimony, it seems most likely that readers typically acquire the visual information necessary for reading during the first 50$70 \mathrm{~ms}$ of a fixation, but when one word changes to another they are aware of it. Both studies are consistent in providing evidence against the notion that there is a serial letter-by-letter scan within a fixation. The same conclusion was reached in a study by Inhoff, Pollatsek, Posner, and Rayner (1989), in which readers read text that was transformed in some way. By requiring readers to read mirror-image text (or rotated or reversed text) and varying (a) the direction of letters within words (left to right vs. right to left) and (b) the direction of reading (left to right vs. right to left), Inhoff et al. showed that congruency of letter order and direction of reading is not critical for reading (Kowler \& Anton, 1987) and that a serial scan of letters in foveal vision does not occur.

\section{The Perceptual Span in Reading}

How much useful information can a reader obtain during eye fixations? Many different techniques have been used to estimate the size of the effective visual field or perceptual span in reading. However, most of them have severe limitations that are not discussed here (see Rayner, 1975b, 1978b for discussion). Rather, the focus is on studies using eye-contingent display tech-

\footnotetext{
${ }^{4}$ Ishida and Ikeda (1989) concluded that the visual sensitivity that is suppressed during a saccade recovers only partially during the initial part of a fixation and that it is fully recovered approximately $70 \mathrm{~ms}$ after the beginning of the fixation.
} 
during a saccade because the eyes are moving so *

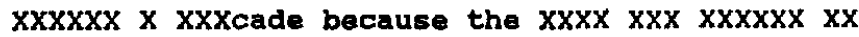
*

$\operatorname{XXXXXX} \times \operatorname{XXXXXXX} \mathrm{XXXXXse}$ the eyes are $\operatorname{mXXXXX} \mathrm{XX}$

during a saccade $\underset{\star}{\operatorname{xxx}} \operatorname{xxx}$ the eyes are moving so

during a saccade becausXXXXXXXYes are moving so *

during a saccade because the dogs are moving so

during a saccade because the eyes are moving so
Normal Text

Moving Window

Foveal Mask

Boundary

Figure 2. Examples of the moving window, foveal mask, and boundary paradigms. The first line shows a normal line of text with the fixation location marked by an asterisk. The next two lines show an example of two successive fixations with a window of 17 letter spaces (with other letters replaced with $X \mathrm{~s}$ and the spaces between words preserved). The next two lines show an example of two successive fixations with a 7-letter mask. The bottom two lines show an example of the boundary paradigm. The first line shows the text prior to a display change with fixation locations marked by asterisks. When the reader's eye movement crosses an invisible boundary location (the letter $e$ in the), an initially displayed word (dogs) is replaced by the target word (eyes). The change occurs during the saccade so that the reader is not aware of the change.

niques (see Figure 2) developed by McConkie and Rayner (1975) and Rayner (1975b). This research has provided the most definitive information concerning the perceptual span.

In the moving window technique (McConkie \& Rayner, 1975), the text is perturbed except in an experimenter-defined window region around the point of fixation. Wherever the reader looks, the text is visible, while outside of the window area the text is perturbed in some way. Readers are free to move their eyes whenever and wherever they wish, but the amount of useful information that is available on each fixation is controlled by the experimenter. Each time the eyes move, a new region of text is exposed while the region previously fixated is perturbed. In some cases, the window is defined in terms of letter spaces, whereas, in other cases, the window coincides with word boundaries. Sometimes the spaces between words outside of the window are preserved, and other times they are filled in, and sometimes the text is perturbed outside the window only on selected fixations. The assumption with this technique is that when the window is as large as the region from which the reader can obtain information, there is no difference between reading in that situation and when there is no window.

The moving mask technique (Rayner \& Bertera, 1979) is like the moving window technique except that, wherever the reader fixates, a mask obscures the text around fixation while the normal text is presented beyond the mask region. Just as with the moving window technique, the size of the mask can be varied. ${ }^{5}$ In the boundary technique (Rayner, 1975b), a single critical target word is initially replaced by another word or by a nonword. When the reader's saccade crosses over an invisible prespecified boundary location in the text, the initially displayed stimulus is replaced by the target word. The assumption with this technique is that if a reader obtains information from the initially presented stimulus, any inconsistency between what is available on the fixation after crossing the boundary and with what was processed on the prior fixation (when information about the initial stimulus was processed) is registered in the fixation time on the target word. In a related technique (McConkie \& Zola, 1979), a single word in a given target location alternates between two different words with each saccade. Finally, in another variation of the boundary technique (McConkie \& Hogaboam, 1985), text is masked (or simply removed) following a saccade and the reader must report the last word that was read.

Studies using these techniques (DenBuurman, Boersma, \& Gerrissen, 1981; Ikeda \& Saida, 1978; McConkie \& Hogaboam, 1985; McConkie \& Rayner, 1975; O'Regan, 1979, 1980; Pollatsek et al., 1986; Rayner, 1975b, 1986; Rayner \& Bertera, 1979; Rayner et al., 1981; Rayner, Well, Pollatsek, \& Bertera, 1982; N. R. Underwood \& McConkie, 1985; N. R. Underwood \&

\footnotetext{
${ }^{5}$ This situation creates an artificial foveal scotoma and eye movement behavior if the situation is quite similar to the eye movement behavior of patients with real scotomas (Whittaker, Cummings, \& Swieson, 1991; Zihl, 1995).
} 
Zola, 1986) have been very consistent in indicating that the size of the perceptual span is relatively small; for readers of alphabetical orthographies (e.g., English, French, and Dutch) the span extends from the beginning of the currently fixated word but no more than 3-4 letters to the left of fixation ${ }^{6}$ (McConkie \& Rayner, 1976a; Rayner, Well, \& Pollatsek, 1980; N. R. Underwood \& McConkie, 1985) to about 14-15 letter spaces to the right of fixation (DenBuurman et al., 1981; McConkie \& Rayner, 1975; Rayner, 1986; Rayner \& Bertera, 1979; Rayner et al., 1981). ${ }^{7}$ Thus, the span is asymmetric to the right for readers of English. However, for orthographies, such as Hebrew, that are printed from right to left, the span is asymmetric to the left of fixation (Pollatsek, Bolozky, Well, \& Rayner, 1981).

Characteristics of the writing system influence not only the asymmetry of the span but also the overall size of the perceptual span. Ikeda and Saida (1978) found that the perceptual span for readers of Japanese was about 13 character spaces ( 6 character spaces to the right of fixation ), which is considerably smaller than that of readers of English. Osaka $(1987,1992)$ found that the span was larger for text consisting of a combination of kanji (an ideographic script) and kana (a phonetic-based script) than for text consisting only of kana characters. For the combined kana-kanji script (which is typical of Japanese text), the span extended 7 character spaces to the right of fixation, whereas for the kana script it extended 5 character spaces to the right of fixation. These studies involved horizontal reading, but Japanese can also be printed (and read) vertically. Osaka and Oda (1991) found that the span was 5-6 character spaces in the vertical direction of the eye movement. Subsequent experiments by Osaka (1993a) revealed that the span was asymmetric when reading horizontally and vertically. Osaka (1993b) also found (using the moving mask technique) that readers of Japanese had a great deal of difficulty when 4 or 6 characters in the center of vision were masked; this result is quite consistent with results with readers of English (Rayner \& Bertera, 1979), except that the mask needed to be larger for English to cause the same type of disruption (which is consistent with the finding that more characters are processed per fixation in English than in Japanese). Recently, Inhoff and Liu (1998) found that readers of Chinese have an asymmetric perceptual span extending from 1 character space left of fixation to 3 character spaces to the right. In addition to the characteristics of the writing system influencing the size of the perceptual span, reading skill influences it. Rayner (1986) found that beginning readers had a smaller span (about 12 letter spaces to the right of fixation) than skilled readers (14-15 letter spaces) but that it was asymmetric to the right of fixation.

The results of a number of studies are quite consistent in indicating that, for skilled readers of alphabetic writing systems, the perceptual span extends about 14-15 character spaces to the right of fixation. However, this does not mean that words can be identified that far from fixation; indeed, word length information is acquired further to the right of fixation than is letter information (Ikeda \& Saida, 1978; McConkie \& Rayner, 1975; Rayner, 1986). The word identification span (or area from which words can be identified on a given fixation) is smaller than the total perceptual span (Rayner et al., 1982; McConkie \&
Zola, 1987; N. R. Underwood \& McConkie, 1985) and generally does not exceed 7-8 letter spaces to the right of fixation.

Is the size of the perceptual span better thought of in terms of letters or words? The answer appears to differ to the left and right of fixation. Rayner, Well, and Pollatsek (1980) found that the left boundary of the span was primarily defined by the beginning of the fixated word (though it did not extend more than 4 letter spaces to the left of fixation). In contrast, Rayner et al. (1982) found that the right boundary of the span was primarily defined in terms of letters. When they compared reading performance when the window was defined in terms of the number of letters available to the right of fixation with reading performance when the window was defined by the number of words to the right of fixation, there was no difference between "word" and "letter windows" when they were roughly equivalent in size. More critically, detailed analyses revealed that performance in the word window conditions (in which word integrity was maintained) could be predicted very accurately from knowing the number of letters available on each fixation, whereas performance in the letter window conditions could not be predicted from knowing the number of words available on each fixation. Thus, the perceptual span should be defined in terms of the number of letters available to the right of fixation.

Another interesting issue is whether or not readers are able to acquire information from below the line that they are reading. Inhoff and Briihl (1991; Inhoff \& Topolski, 1992) addressed this issue by asking readers to read a line from a target passage while ignoring a distracting line (from a related passage) as their eye movements were recorded. When readers completed a line, they pushed a button, and the next line (with the corresponding distracting line of text directly below the target text) was presented. Readers' answers to multiple-choice questions suggested that they had obtained information from both attended and unattended lines. However, a detailed examination of their eye movements showed that they occasionally fixated unattended text. When such fixations were excluded, there was no indication that readers obtained useful semantic information from the unattended text. Pollatsek, Raney, LaGasse, and Rayner (1993) more directly examined the issue by using a moving window technique. The line the reader was reading and all lines above it were normal, but the text below the currently fixated line was altered. The lines below the currently fixated line consisted of (a) the original text, (b) lines from another passage (a semantically different condition), (c) Xs, (d) visually similar letters, or (e) dissimilar letters replacing the letters from the original text. Pollatsek et al. found that the passages were read most easily when the normal text was below the line and when there were $X \mathrm{~s}$ below the line. None of the other conditions

\footnotetext{
${ }^{6}$ Binder, Pollatsek, and Rayner (in press) recently found that there are some circumstances under which readers process more information to the left of fixation. For example, when a word is skipped, attention may often be directed to the left of the fixation following the skip.

${ }^{7}$ It is important to note that the moving window technique yields a maximum span estimate rather than an absolute value for each fixation (Well, 1983). Thus, although the size of the perceptual span has been estimated as being 14-15 letter spaces to the right of fixation, there is variability in the size of the actual perceptual span from fixation to fixation, as some studies reviewed in a later section have demonstrated.
} 
differed from each other, which suggests that readers do not obtain semantic information from below the line of text. However, when the task was to find a target word in the passage, readers were occasionally able to obtain information below the fixated line (see also Prinz, 1984).

A final important finding is that the size of the perceptual span is not constant but varies as a function of text difficulty (Inhoff et al., 1989; Rayner, 1986). Rayner found that the size of the span was smaller when text was difficult to read. When fourth-grade children were given reading-level-appropriate text, the size of their perceptual span was similar to that of adult readers. However, when they were given college-level text, their span became much smaller.

In summary, it appears that the perceptual span for readers of English generally extends from 3-4 letter spaces to the left of fixation to about 14-15 spaces to the right. When the reading material is difficult, the size of the span tends to be smaller than this. Typically, readers focus their attention on the currently fixated line, and information below the line of text is not obtained, unless the task (e.g., visual search) or characteristics of the orthography demand it (e.g., reading vertically arranged ideographic systems such as Chinese or forms of Japanese). For readers of languages printed from right to left, the perceptual span is asymmetric to the left of fixation (Pollatsek et al., 1981). The size of the span also varies as a function of orthography and is smaller for more densely packed writing systems.

\section{Acquiring Information to the Right of Fixation}

What kind of information is acquired to the right of fixation when reading English? Rayner et al.'s (1982) finding that individual letters to the right of fixation are more critical than word integrity and that the acquisition of letters is not tied to words being intact suggests that readers acquire partial word information from the parafovea. Further evidence for this conclusion comes from another experiment reported by Rayner et al. (1982), in which readers read text when (a) only the fixated word was available and all other letters to the right of fixation were replaced by another letter (a one-word window), (b) the fixated word and the word to the right of fixation were available and all other letters were replaced by another letter (a two-word window), or (c) the fixated word as well as partial information about the word to the right of fixation were available. In the third condition, 1,2 , or 3 letters of the word to the right of fixation were available on each fixation. When the first 3 letters of the word to the right of fixation were available and the remainder of the letters were replaced by visually similar letters, reading rate was not too different from when the entire word to the right was available; if the remainder of the word was replaced with visually dissimilar letters, reading was not as good as when the word to the right was available, suggesting that more information is often processed than just the 3 letters at the beginning of the next word. Lima and Inhoff (1985) and Lima (1987) also found that the time a reader looked at a word decreased when the first 3 letters of a word were available on the prior fixation (compared to when they were not available on the prior fixation).

Why are the first 3 letters of the next word to be fixated important in facilitating reading? One obvious reason is that they are used in initiating lexical access processes. Another possibility is that they are important because they are closer to fixation than the letters further into the next word. That this is not the cause of the facilitation is clear from experiments by Inhoff (1987, 1989a, 1989b, 1990; Inhoff, Bohemier, \& Briihl, 1992; Inhoff \& Tousman, 1991), in which words were either printed normally from left to right or in reversed order from right to left (but with letters within the words printed normally from left to right). As readers fixated on word $n-1$, either the entire target word was available or the first 3 letters of the target word were available (with the other letters being replaced by $X$ s, visually similar, or visually dissimilar). Inhoff found that readers obtained considerable facilitation when the first 3 letters of the parafoveal word were available prior to fixating it when reading from left to right. More importantly, he also found that reading rate was facilitated by having the first 3 letters of the target word available when reading from right to left (where the beginning letters of the word were further away from fixation than the end letters). He also found evidence that readers obtain information about the last 3 letters of 6-letter words. When the letters where replaced by $X s$ or dissimilar letters, there was facilitation from having the end letters parafoveally available. However, when the letters were replaced with similar letters, there was virtually no facilitation. Inhoff's results thus show that proximity to the fixation point is not the reason that the initial letters of unidentified parafoveal words are important; rather, they are important because they are useful in initiating lexical access processes, in integrating information across fixations, or in both (see the next section). Although McConkie, Zola, Blanchard, and Wolverton (1982) reported an experiment that led them to conclude that information used to identify a word is obtained only on the fixation on which the word is completely identified, several experiments have found significant effects of partial-word information (Balota, Pollatsek, \& Rayner, 1985; Henderson \& Ferreira, 1990; Inhoff, 1989a, 1989b; Lima, 1987; Lima \& Inhoff, 1985; Pollatsek et al., 1986; Rayner et al., 1982). Thus, the use of partial-word information from the word to the right of fixation is likely to be a general phenomenon.

In addition to partial-word information being obtained parafoveally, word-length information is acquired parafoveally and used in computing where to look next (O'Regan, 1979, 1980; Morris, Rayner, \& Pollatsek, 1990; Pollatsek \& Rayner, 1982; Rayner, 1979a; Rayner \& Morris, 1992; Rayner, Sereno, \& Raney, 1996). It is clear from the fact that readers skip some words (S. F. Ehrlich \& Rayner, 1981; O'Regan, 1979, 1980; Rayner \& Well, 1996) that sometimes words to the right of fixation can be fully identified and skipped. In such cases, the duration of the fixation prior to the skip is inflated (Hogaboam, 1983; Pollatsek et al., 1986). Word-length information may be used not only in determining where to fixate next but also in how parafoveal information is used. That is, enough parafoveal letter information may be extracted from short words so that they can be identified and skipped, whereas partial-word information (the first 3 letters) extracted from longer parafoveal words may rarely allow full identification of them but facilitate subsequent foveal processing. An experiment by Blanchard, Pollatsek, and Rayner (1989) documented this pattern. A parafoveal preview enabled readers to skip short words ( $1-3$ letters) significantly more of 
the time, whereas it had a negligible effect on how often long words (6-10 letters) were skipped. On the other hand, preview shortened the gaze durations on the long words but had a much smaller effect on the gaze durations on the short words.

To what extent do parafoveal words influence the processing of the fixated word? The evidence on this issue is somewhat mixed. Kennedy (1998); Inhoff, Briihl, and Starr (1998); and Murray (1998; Murray \& Rowan, 1998) have reported that the characteristics of the word to the right of fixation can influence the processing of a fixated word. For example, Murray (1998) found that when readers were fixated on the end of word $n$, if word $n+1$ resulted in an implausible reading, the fixation on word $n$ was inflated even when word $n+1$ was subsequently fixated. On the other hand, other studies (Carpenter \& Just, 1983; Henderson \& Ferreira, 1993; Rayner, Fischer, \& Pollatsek, 1998) have found that the frequency of the word to the right of fixation does not influence the processing of the fixated word; fixation time on word $n$ did not vary when word $n+1$ was either a low- or high-frequency word.

\section{Integration of Information Across Saccades}

The extraction of partial-word information from the parafovea implies that it must be integrated in some way with foveal information from the subsequent fixation. When we read, we do not have the experience of seeing text for about $250 \mathrm{~ms}$ followed by a gap or blank period (resulting from the saccade) and then by new text around our new fixation. Rather, the brain smooths out the discrete inputs so that we maintain a stable coherent view of the text we are reading.

What kind of information is integrated across saccades? Important data on thís question have been obtained both from reading studies (with fixation time on a target word as the primary dependent variable) and from a word-naming task introduced by Rayner (1978c). This naming task ${ }^{8}$ is actually a variation of the boundary paradigm discussed earlier. In the task, during an initial fixation, a word or letter string is presented parafoveally. When the participant makes an eye movement to the parafoveal stimulus, it is replaced by a word, which must be named as rapidly as possible. Integration is assessed by examining the effect of the parafoveal stimuli on naming time. These naming studies (Balota \& Rayner, 1983; McClelland \& O'Regan, 1981; Rayner, 1978c; Rayner et al., 1978; Rayner, McConkie, \& Zola, 1980) have been very influential in assessing what type of information is integrated across saccades, but it is important to note that virtually identical results have been obtained from reading studies.

The basic finding from the naming task is that if the first 23 letters of the parafoveal word are the same as for the foveal word (which is present following the saccade), naming is facilitated when the word is presented either $1^{\circ}, 2.3^{\circ}$, or $3^{\circ}$ from fixation (either to the left of fixation or the right); if the parafoveal stimulus is presented $5^{\circ}$ from fixation, then there is no facilitation. When the parafoveal stimulus is presented $1^{\circ}$ from fixation, if the first 2-3 letters are preserved across the saccade, naming is much faster than when these letters change across the saccade but not as fast as when the entire target word is presented parafoveally (and all letters remain the same during the saccade). However, when the parafoveal stimuli are presented $2.3^{\circ}$ or $3^{\circ}$ from fixation, there is no difference between the condition in which the first 2 or 3 letters are preserved across the saccade and the condition in which all letters are preserved. It was suggested (McClelland \& O'Regan, 1981; Paap \& Newsome, 1981) that the basic pattern of results is obtained only when there is a limited target set, but Balota and Rayner (1983) demonstrated the robustness of the effect when a large stimulus set was used.

Much of the original interest in the integration of information across saccades was related to the notion of an integrative visual buffer in reading (McConkie \& Rayner, 1976b; Rayner, 1978c) in which gross visual information obtained from a parafoveal word was stored in a buffer and then combined with information available in the fovea about that word following the saccade. According to McConkie and Rayner (1976b), the justification of the information from the two fixations could be based on (a) knowledge about how far the eyes moved and (b) the commonality of the visual patterns. Although an integrative visual buffer has a certain amount of intuitive appeal, a number of experiments found no support for it. First, Rayner et al. (1978) found that the typical pattern in the naming task could be obtained when the saccade was simulated. In the simulation condition, the viewer maintained fixation, the parafoveal stimulus was presented for a period of time approximating the latency of a saccade, and then the target word appeared in foveal vision for the viewer to name. Thus, information initially impinged on the parafoveal retina and then on the fovea, just as it did when a saccade was made. The fact that the same pattern of results was obtained under both conditions suggests that knowledge about how far the eyes moved is not necessary for integration. In addition, Lévy-Schoen and O'Regan (1979) and McConkie, Zola, and Wolverton (1980; see also McConkie \& Zola, 1987) found that readers are unable to detect small shifts of text during saccades; if the movement of the text was no larger than one fourth the size of the saccade, they were unaware that anything odd occurred (though they did sometimes make small corrective saccades to adjust for the movement).

The finding that the visual display can be shifted a small amount during the saccade without the reader noticing it is problematic for an integrative visual buffer. As indicated above, this result suggests that knowledge of how far the eyes moved is not crucial for integration. Thus, integration would have to be based purely on the commonality of visual patterns from two fixations, and how to align the two images might be difficult. Even more problematic for the integrative visual buffer notion were subsequent experiments that demonstrated that case changes did not affect reading (McConkie \& Zola, 1979; Rayner, McConkie, \& Zola, 1980). McConkie and Zola had readers read text in alternating case, and each time they moved their eyes, the text shifted from one version of alternated case to another (e.g., $c H a N g E$ shifted to $C h A n G e$ ). Readers did not notice that the change was taking place, and their reading behavior was not different from a control condition in which they read alternated case in which the letters did not change case from fixation to fixation. If visual codes were important in

\footnotetext{
${ }^{8}$ Other tasks (such as lexical decision, same-different matching, and categorization judgments) have been used, but the bulk of the studies have used naming.
} 
integrating information across saccades, then the change of features between upper- and lowercase letters should have disrupted reading. Thus, visual information is not combined across saccades during reading in an integrative visual buffer.

A number of other candidates for the code conveying information across saccades have been considered. One candidate is some type of phonological code. Pollatsek, Lesch, Morris, and Rayner (1992) found in both the naming task (see also Henderson, Dixon, Petersen, Twilley, \& Ferreira, 1995) and reading that phonological information is used in integrating information across saccades. In Pollatsek et al.'s (1992) study, a homophone of a target word presented as a preview in the parafovea facilitated processing of the target word seen on the next fixation more than a preview of a word matched with the homophone in orthographic similarity to the target word. Because the orthographic similarity of the preview to the target also plays a part in the facilitative effect of the preview, however, codes other than phonological codes ( such as abstract letter codes) are preserved across saccades.

Morphemes have also been examined as candidates for facilitating the integration of information. Lima (1987) examined whether prefixes could be extracted as a unit from the beginning of a parafoveal word. She used words that had true prefixes (e.g., revive) and words that were "pseudoprefixed"' (e.g., rescue). If extracting morphemes were a significant part of the benefit of parafoveal preview, then a larger preview benefit (the difference in performance between when a parafoveal preview of the target was present and when the preview was absent) should be observed for the prefixed words. There was equal benefit in the two cases, which suggests that prefixes are not active units in integration across saccades. Another morphemic unit that could be extracted from the parafovea is the first part of a compound word. Inhoff (1989a) compared the preview benefit from seeing the first morpheme of a compound word such as cow in cowboy with that from the first part of a "pseudocompound" word, such as car in carpet, and found no difference between the two conditions.

Finally, it has been suggested that semantic preprocessing of unidentified parafoveal words is a relevant factor in integration across saccades and aids in the later identification of a word (G. Underwood, 1985). Rayner, Balota, and Pollatsek (1986) tested the idea that semantic preprocessing influences subsequent fixation time in a boundary study. Prior to fixating on a target word (such as tune), the parafoveal preview for that word was either orthographically similar (turc), semantically similar (song), or unrelated (door). When the eyes crossed the boundary location, the target word replaced the preview word. The semantically similar pairs (song-tune) produced a priming effect in a standard priming experiment. However, in the reading situation, although fixation time on the target word was shorter when the preview was orthographically similar to the target word, there was no difference between the semantically similar and unrelated conditions. Thus, readers apparently do not obtain semantic information from unidentified parafoveal words.

In summary, it appears that abstract letter codes (McConkie \& Zola, 1979; Rayner, McConkie, \& Zola, 1980) and phonological codes (Henderson et al., 1995; Pollatsek et al., 1992) are involved in integrating information from words across saccades.
No evidence has been obtained to suggest that visual, morphological, or semantic information is important.

\section{The Effect of Lexical and Sentential Constraint}

To determine whether lexical constraint can influence parafoveal processing, Lima and Inhoff (1985) presented sentences in which one of two words appeared in a target location. The target words, for example dwarf and clown, were selected to have equal frequency in the language and be equally predictable in the sentence context. However, the initial letters of the target word in one condition ( $d w a$ ) were shared by few words in the lexicon, whereas those in the other condition (clo) were shared by many words. Because prior studies had demonstrated that seeing the first three letters of the parafoveal word produced a large benefit, Lima and Inhoff reasoned that if lexical constraint were a potent variable in parafoveal processing, then the preview benefit for $d w a r f$ should be greater than for clown. In fact, there was equal preview benefit in the two cases, indicating that lexical constraint does not operate on parafoveal information. However, the fixation time on clown was actually less than for $d w a r f$ (regardless of whether there was a preview or not), indicating that the frequency of the word-initial-letter sequence influences the time to process a word foveally. In a related vein, Inhoff and Rayner (1986) found that more parafoveal preview benefit was obtained from a high-frequency parafoveal word than from a low-frequency parafoveal word.

The effect of sentential constraint on parafoveal processing was examined by Balota et al. (1985). They varied both the predictability of a target word and the availability of parafoveal information by using the boundary technique. Two interesting findings emerged. First, the finding that a predictable target word is more likely to be skipped than an unpredictable one (S. F. Ehrlich \& Rayner, 1981; O'Regan, 1979) was replicated indicating that sentential constraint influences the usefulness of parafoveal information. Second, when the target word was not skipped, fixation time on it was shorter when the word was more predictable. More importantly, the benefit of a parafoveal preview was greater when the target word was predictable, indicating that the extraction of parafoveal information is more efficient when aided by sentential context. Additional analyses indicated that more letters were extracted from the parafovea when contextual constraint was high.

An interesting corollary to the Balota et al. (1985) study is that, when the difficulty associated with processing the foveal word is high, the extraction of parafoveal information decreases (Henderson \& Ferreira, 1990; Inhoff et al., 1989; Kennison \& Clifton, 1995; Rayner, 1986; Schroyens, Vitu, Brysbaert, \& d'Ydewalle, in press). For example, Henderson and Ferreira (1990) used the boundary technique and manipulated foveal processing difficulty while also varying the availability of parafoveal information. They found that difficult foveal processing led to the reader obtaining no parafoveal preview from the word to the right of fixation. These results thus suggest that the amount of information processed on a fixation is somewhat variable and can be influenced by factors such as the length of the currently fixated word and the word to the right of fixation (Rayner, 1979a), sentential context (Balota et al., 1985), and the difficulty of processing the fixated word (Henderson \& Ferreira, 1990). 


\section{Display Change Effects?}

A final issue to be addressed in this section is the extent to which the results of studies using eye-contingent display changes are due to the display changes per se. Each time a display change occurs in such studies, because of phosphor persistence of letters on the display monitor or the refresh rates of the display monitor (or both), there is a flash on the screen from replacing letters by other letters. ${ }^{9}$ One could argue (see O'Regan, 1990, 1992) that the change per se is somehow influencing the results or that the amount of change artifactually influences the data pattern so that the more letters that are replaced, the more disruption there will be, or, given that the number of letters that change is held constant, either the location of the changing letters (closer or further from the fixation) or the characteristics of the changing letters (replacing letters with visually similar letters might cause less of a flash than replacing letters with dissimilar letters) might influence the results.

To what extent is this issue a worry? Some of the earliest experiments of the genre (McConkie \& Rayner, 1975; Rayner, 1975b) suffered from the fact that the display changes associated with eye movements were relatively slow, so readers undoubtedly saw some of the letters change. The eye-tracking and computer systems currently used in eye-contingent change experiments (see Beauvillain \& Beauvillain, 1995; McConkie, Wolverton, \& Zola, 1984; McConkie, Wolverton, Zola, \& Burns, 1978; Rayner, 1979b; van Diepen, DeGraef, \& Van Rensbergen, 1994) are more sophisticated than those used in the early classic experiments, and the more recent research with faster display changes have yielded patterns of data very consistent with those originally reported. The currently used display change techniques generally mean that in moving window experiments the movement of the window is completed within $3-8 \mathrm{~ms}$ of the end of the saccade ${ }^{10}$ and the speed of the display change in studies using the boundary technique guarantees that the change occurs during the saccade. In the classic McConkie and Rayner (1975) experiment, it was argued that the perceptual span extended 15 letter spaces to the right of fixation. Although there were lingering effects for windows larger than 15 letter spaces to the right of fixation, McConkie and Rayner argued that such effects (which were not significant) were due to readers seeing movement in parafoveal vision (as a result of the display change). As this review has documented, many subsequent experiments (with faster display changes) have found the size of the window to be about 15 letter spaces to the right of fixation. Likewise, Pollatsek et al. (1986) obtained results that were quite consistent with those of Rayner (1975b).

Another issue to consider is that readers in moving window and boundary experiments are often not aware that display changes are taking place. If there are $X \mathrm{~s}$ outside of a moving window, readers are certainly aware that the $X \mathrm{~s}$ are there, because they represent a homogeneous pattern. However, if the letters outside the window are replaced with other letters and readers have approximately $7-8$ normal letters to the right of fixation, they are unaware of the display changes. Even when the window is smaller than this and visually similar letters are outside of the window, readers are aware of reading more slowly than usual, but they do not know why. In boundary experiments, if the display change is slowed down so that readers can see the change take place, they are aware of changing letters. However, under normal experimental conditions, even though they are encouraged to report any strange events as they read, they rarely do so, and even skilled observers who know what is happening in the experiment do not perceive the changes.

It could be argued that it does not matter whether or not readers are aware of the display changes, because there is a change in the stimulus pattern and it is registered by the visual system (even when the change occurs during a saccade) and thus interacts with the manipulations being made. If this is so, then the interaction is very complex and not easily interpretable. The clearest example of this is the situation in which the display change is constant (in terms of the number of letters changing) but some other variable, such as contextual constraint, is varied and is found to interact with the amount of parafoveal information obtained (Balota et al., 1985). Another factor is that it has consistently been found that $X$ s outside the window lead to better performance than when letters are outside of the window. This is undoubtedly due to readers trying to incorporate erroneous letters into their reading in the latter case. However, a "flicker" explanation of the results should lead to the opposite prediction, because there would be more of a flash going from $X \mathrm{~s}$ to the normal text than in going from letters to other letters. Although one always has to worry about extraneous factors influencing performance, the evidence in favor of display change artifacts seems quite limited. Indeed, Briihl and Inhoff (1995) and Inhoff, Starr, Liu, and Wang (1998) directly tested the idea that display changes per se influence the outcome of eye-contingent display change studies. They varied the speed of a display change and the refresh rate of the display monitor and found - no evidence to suggest that the results of eye-contingent change experiments were artifacts of the paradigm.

\section{The Control of Eye Movements in Reading}

One factor that dampened interest in using eye movements to study reading at the end of the second era of eye movement

\footnotetext{
${ }^{9}$ There is some controversy over the exact amount of phosphor persistence due to different types of phosphors (see Di Lollo, Bischof, WaltherMuller, Groner, \& Groner, 1994; Groner, Groner, Muller, Bischof, \& Di Lollo, 1993; Irwin, 1994; Westheimer, 1993). Much of the controversy centers on how much persistence remains when a letter is removed from a monitor. However, in most experiments discussed in this section, one letter is replaced by another letter, and readers do not have the impression of overlapping letters. In addition, a number of studies (Kennedy \& Baccino, 1995; Kennedy, Brysbaert, \& Murray, 1998; Kennedy \& Murray, 1991, 1993, 1996; Neary \& Wilkins, 1989; Wilkins, 1986) have shown that eye movement control is disturbed by pulsating illumination characteristic of display monitors that are periodically refreshed. In particular, the size of a saccade varies as a function of refresh rate. However, this finding is not directly relevant to the issue under discussion because across experiments in which display changes take place, the refresh rate is constant across the experimental conditions.

${ }^{10}$ There is some variability across labs that use eye-contingent display changes in terms of how quickly the display change is made. Thus, attention should be paid to what each investigator reports as to how quickly the change can be made. It should also be noted that some labs use an algorithm whereby the location of the next saccade can be predicted on the basis of the velocity of the saccade. Also, the computations can be made such that the window moves as the eye is moving and does not depend on knowing exactly where the landing position will be.
} 
research was the belief that eye movements are unrelated to ongoing cognitive processes (Bouma \& deVoogd, 1974; Kolers, 1976; Viviani, 1990). Over the past 20 years, abundant evidence has accumulated indicating that fixation time and saccade length are related to aspects of the text currently fixated (Kapoula, 1984; Rayner, 1997; Rayner, Sereno, et al., 1989). In a following section, research related to ongoing language processing is reviewed. In this section, the focus is on the extent to which how far the eyes move and how long the eyes fixate are determined by the information currently fixated.

The earliest unambiguous demonstration that the length of the next saccade and the duration of the current fixation are computed on-line was provided by Rayner and Pollatsek (1981). They varied the physical aspects of the text randomly from fixation to fixation and found that the behavior of the eyes mirrored what was seen on a fixation. In one experiment, the size of the window of normal text was randomly varied from fixation to fixation, and saccade length was shown to vary accordingly. In another experiment, the foveal text was delayed after the onset of a fixation by a mask (with the time varying randomly from fixation to fixation), and fixation durations varied accordingly ( see also Morrison, 1984). In addition, the manipulations appeared to affect saccade length and fixation duration independently. Thus, there is reason to believe that the decisions of where and when to move the eyes can be made independently (Rayner \& McConkie, 1976). Accordingly, these two topics are discussed separately.

\section{Where to Move the Eyes}

What influences where to move the eyes next in reading? As indicated above, one important influence on where to move is the amount of useful letter information to the right of fixation. Balota et al. (1985) demonstrated that letter information to the right of fixation was important in determining whether a word was skipped. However, most of the research suggests that word boundary information (conveyed by the spaces between words) is the major determinant used in deciding where to move to next; saccade length is influenced by both the length of the fixated word and the word to the right of fixation (O'Regan, 1979, 1980; Rayner, 1979a). Such a relationship, however, could also be a result of the linguistic characteristics of words (because shorter words tend to have a higher frequency in the language). In order to examine the independent effect of word boundary information, text has been presented to readers in which all of the letters to the right of the window boundary were replaced by $X s$ but the spaces were preserved. This condition was compared to one in which the spaces were also replaced by Xs (McConkie \& Rayner, 1975; Rayner, 1986). Although mean saccade length decreased somewhat when the letter information to the right of fixation was removed, the removal of space information more seriously affected saccade length. Pollatsek and Rayner (1982) also showed that saccade length decreased markedly when the spaces between words were filled in, even when the letters were left intact. These experiments suggest that letter information may be of little value in guiding the eye if word boundary information is not present. That word length information is important in eye guidance is clear from an experiment by Morris et al. (1990) in which the only useful information to the right of the fixated word was the space information delimiting word $n+1$ (all the letters to the right of word $n$ were converted to $X s$ ). Despite the fact that word $n+$ 1 was all $X$ s before it was fixated, readers made a larger saccade off word $n$, the longer word $n+1$ was.

Although most researchers have found evidence that word length is an important cue in deciding where to look next, Epelboim, Booth, and Steinman (1994, 1996; see also Epelboim, Booth, Ashkenazy, Taleghani, \& Steinman, 1997) argued that word length per se is not critical for eye guidance. On the basis of experiments in which readers read text with the spaces removed, Epelboim et al. claimed that reading unspaced text is relatively easy. They further argued that the elimination of space information between words primarily disrupts the word recognition process. Subsequent analyses by Rayner and Pollatsek (1996) demonstrated that most readers are slowed down (on average by about $30 \%$ ) by the absence of space information (see also Spragins, Lefton, \& Fisher, 1976), and experiments by Rayner, Fischer, and Pollatsek (1998) demonstrated that both word identification processes and eye guidance are disrupted by the lack of space information (Pollatsek \& Rayner, 1982). Rayner et al. (1998) found that (a) the initial landing position in words shifted closer to the beginning of a word and (b) lowfrequency words were harder to identify than high-frequency words when space information was absent. In another recent demonstration, it was found that, when space information is provided for readers of Thai (who are not used to reading with spaces between words), they read more effectively than normal (Kohsom \& Gobet, 1997). Likewise, Inhoff, Radach, and Heller (1996) reported that the reading of long German compound words is facilitated by the insertion of spaces, even though this format is ungrammatical and never encountered in normal reading. Thus, it appears safe to conclude that space information is used for guiding the eyes in reading.

Word-length information also plays a clear role in where in the word a reader fixates. Although there is variability in where the eyes land on a word, readers tend to make their first fixation on a word about halfway between the beginning and the middle of a word (Dunn-Rankin, 1978; McConkie, Kerr, Reddix, \& Zola, 1988; McConkie, Kerr, Reddix, Zola, \& Jacobs, 1989; McConkie \& Zola, 1984; O'Regan, 1981; Radach \& Kempe, 1993; Rayner, 1979a; Rayner et al., 1996; Rayner, Fischer, et al., 1998; Vitu, 1991a, 1991b, 1991c; Vitu, O'Regan, Inhoff, \& Topolski, 1995; Vitu, O'Regan, \& Mittau, 1990). Rayner (1979a) originally labeled this prototypical location as the preferred viewing location. Subsequently, O'Regan and LévySchoen (1987) distinguished between the preferred viewing location and what is now referred to as the optimal viewing position. ${ }^{11}$ The optimal viewing position is the location in a word at which recognition time is minimized. According to O'Regan and Lévy-Schoen (1987), the optimal viewing position is a bit to the right of the preferred viewing location, closer to the center of the word. Extensive research efforts have examined the consequences of making fixations at locations other than this optimal viewing position (Coëffé, 1985; Holmes \& O'Regan,

\footnotetext{
${ }^{11}$ O'Regan (1981) originally called the optimal viewing location the convenient viewing position.
} 
1987; Nazir, 1991, 1993; Nazir, Heller, \& Sussmann, 1992; Nazir, O'Regan, \& Jacobs, 1991; O'Regan \& Jacobs, 1992; O'Regan, Lévy-Schoen, Pynte, \& Brugaillère, 1984; G. Underwood, Clews, \& Wilkinson, 1989; Vitu, 1991c, 1993; Vitu \& O'Regan, 1988, 1991; Vitu et al., 1990). For words presented in isolation, two general effects have been found. First, there is a refixation effect: The further the eyes are from the optimal viewing position, the more likely it is that a refixation will be made on the word. Second, there is a processing-cost effect: For every letter that the participant's fixation deviates from the optimal viewing position, the associated cost amounts to about $20 \mathrm{~ms}$ (O'Regan et al., 1984). When words are in text, however, although the refixation effect remains, the processing-cost effect is greatly attenuated or absent (Rayner et al., 1996; Vitu et al., 1990). This indicates that contextual information overrides low-level visual processing or that in reading text, the information acquired about a word before it is directly fixated affects its later fixation location and duration.

In any event, there is a clear tendency for readers to fixate between the beginning and the middle of the word. Evidence that word-length information helps to guide the eye toward the preferred viewing location comes from conditions in which there was no parafoveal letter information (Morris et al., 1990). When the space indicating the location of word $n+1$ was visible in the parafovea, the first fixation on that word was closer to the preferred viewing location than when the parafoveal preview did not contain that space information.

A given fixation location in a word can be viewed not only as a landing position but also as the takeoff point or launch site to the next word, and one launch site is related to the next landing position (McConkie, Kerr, et al., 1988; Radach \& Kempe, 1993; Radach \& McConkie, 1998; Rayner et al., 1996). Although the average landing position in a word lies between the beginning and middle of a word, this position varies as a function of the distance from the prior launch site. For example, if the distance to a target word is large (8-10 letter spaces), the landing position is shifted to the left. Likewise, if the distance is small (2-3 letter spaces), the landing position is shifted to the right. To explain the launch site effect, McConkie, Kerr, et al. (1988) suggested that something like a range effect might explain these results ( see also McConkie, Kerr, \& Dyre, 1994). The range effect (Kapoula, 1985; Kapoula \& Robinson, 1986) occurs when the eyes saccade toward isolated targets at various eccentricities: they tend to land near the mean of the range of possible eccentricities instead of directly saccading toward the position of the target. Thus, targets presented at a small or a large eccentricity are overshot and undershot, respectively. However, Vitu (1991b) showed that when words were presented in isolation at different eccentricities from fixation, the eyes' landing position did not differ as a function of eccentricity. Thus, she argued that the range effect cannot account for the launch site data and that a "center of gravity" account (in which readers target the middle of a word for the next saccade) can better explain the data. More recently, Radach and McConkie (1998) provided analyses that are more consistent with a range effect explanation than with a center of gravity account.

The location of the first fixation is between the beginning and the middle of a word for words that are 4-10 letters long (either for the first fixation in a word or when only a single fixation is made). However, with longer words, the effect breaks down (Vitu, 1991a), and readers tend to fixate near the beginning of the word and then make a second fixation toward the end of the word (Hyönä, Niemi, \& Underwood, 1989; O'Regan et al., 1984; Rayner \& Morris, 1992; G. Underwood, Bloomfield, \& Clews, 1988; G. Underwood, Clews, \& Everatt, 1990; G. Underwood, Hyönä, \& Niemi, 1987; Vitu, 1991b). Hyönä et al. (1989); O'Regan et al. (1984); Pynte, Kennedy, and Murray (1991); Rayner and Morris (1992); and G. Underwood et al. (1987, 1988; G. Underwood, Clews, et al., 1990) have shown that informational density (or morphological structure) of the word influences how long the fixations are on each half of the word. For example, Hyönä et al. (1989, using Finnish) found that if the word was predictable from the first 6 letters (the words were typically about 12 letters), readers generally made a fixation in the first half of the word and then moved their eyes to the next word; if they made a second fixation on the word it tended to be quite short. However, if the word could only be identified by knowing what the ending was, readers typically made a short fixation at the beginning followed by a longer fixation on the end of the word.

Although low-level visual information influences where the eyes initially land in a word, it is less clear whether properties other than the spaces between words and the length of a notyet-fixated word are used in deciding where to fixate next. G. Underwood et al. (1987, 1990; Everatt \& Underwood, 1992; Hyönä et al., 1989) examined the landing position in long words (10 or more letters) composed of informative and redundant halves. They reported that the eyes initially move further into a word when the informative information is at the end of the word than at the beginning and suggested that semantic preprocessing of parafoveal words was responsible for the effect. However, neither Rayner and Morris (1992) nor Hyönä (1995a) replicated the effect ( see also Radach, Krummenacher, Heller, \& Hofmeister, 1995). On the other hand, there are demonstrations that orthographic properties of words influence the initial landing position. That is, some studies (Beauvillain \& Doré, 1998; Beauvillain, Doré, \& Baudouin, 1996; Doré \& Beauvillain, 1997; Hyönä, 1995a) have demonstrated that an orthographically irregular letter cluster at the beginning of a word results in the eyes initial landing position deviating toward the beginning of the word, although Radach et al. (1995) did not observe such an effect. Whether or not morphology influences the initial landing position is somewhat unclear. Beauvillain (1996) used prefixed and suffixed French words that were around 10 letters long and found little effect of morphology on initial landing positions. On the other hand, Inhoff, Briihl, and Schwartz (1996) found that the initial fixation was more toward the center of a word for compound words than for derived (or monomorphemic) words. However, they also found that initial fixations on suffixed and monomorphemic words were virtually identical. Hyönä and Pollatsek (in press) varied the length of the initial morpheme of Finnish words, while holding the overall length constant, and found no effect on the initial landing position. They also varied the frequency of the initial morpheme, while holding the overall frequency constant, and obtained a small (about a quarter of a letter position) difference such that the landing position was further into the word for more frequent morphemes. In summary, it is clear that low-level visual factors 
influence where readers fixate next. Furthermore, there appear to be effects of the regularity of the initial letter clusters. However, it is unclear if morphology influences the initial landing position and semantic preprocessing effects do not reliably influence where the eyes land in a word (Liversedge \& Underwood, 1998).

To this point, the focus has been on forward saccades. However, many saccades do not simply move the reader forward from word to word through the text: Sometimes they regress, sometimes they refixate on the fixated word, and sometimes they skip words. Each of these topics is now discussed.

\section{What Causes Regressions?}

Although roughly $10-15 \%$ of all fixations are regressions, very little is really known about what causes them. As noted earlier, text difficulty strongly influences the number of regressions that readers make. Readers are much more likely to regress to a word on the current line than to words on previous lines (Duffy, 1992; K. Ehrlich \& Rayner, 1983). However, when they do regress further back in text, although there are sometimes backtracking movements readers often have fairly good spatial memories for where they went wrong in comprehension and make fairly accurate saccades to that region of text (Frazier \& Rayner, 1982; Kennedy, 1983, 1992; Kennedy \& Murray, 1987a, 1987b). Frazier and Rayner (1982) also demonstrated that when readers encountered a word indicating that their prior interpretation of the sentence was in error, they often made a regression as soon as they encountered disambiguating information. It is likely that many regressions are due to comprehension failures (Blanchard \& Iran-Nejad, 1987; K. Ehrlich, 1983; Frazier \& Rayner, 1982; Hyönä, 1995b; Just \& Carpenter, 1978; Shebilske \& Fisher, 1983; Vauras, Hyönä, \& Niemi, 1992). However, many regressions are very short saccades and are probably due to oculomotor errors. It is interesting that regressive saccades are more likely to occur following longer forward saccades (Vitu, McConkie, \& Zola, 1998); this suggests that long saccades are less accurate and lead to ill-placed fixations. Because regressions are hard to induce experimentally, it is more difficult to examine them than forward saccades.

\section{What Causes Refixations?}

About $15 \%$ of the words in text are refixated (i.e., they receive additional fixations before the reader leaves the word). Why does this happen? O'Regan and Lévy-Schoen (1987; O'Regan et al., 1984) argued that refixations on a word are often caused by originally landing in a "bad" place in a word and that processing of the word is distributed over two or more fixations in such cases. This suggests that (a) most refixations on words should take the eyes to the optimal viewing location and (b) second fixations on a word should take longer than first fixations (because less information would be obtained when the eyes are in a bad place). When readers make two fixations on a word, in the vast majority of cases, an initial fixation on a word is followed by a rightward movement within the word. By far the most frequent pattern is to fixate near the beginning of a word followed by a fixation near the end of the word (Rayner \& Pollatsek, 1987; Rayner et al., 1996). First fixations tend to be longer than the second fixations (Rayner et al., 1996). Thus, although some second fixations on a word are made because the reader was in a bad place, refixations are also often made for other reasons. Certainly, when more than two fixations are made on a word, it is as likely that the additional fixations are as attributable to incomplete lexical processes (Pollatsek \& Rayner, 1990; Pynte, 1996) as to being in a bad place in the word. It is also the case that frequency affects the first fixation of words that are fixated twice (Rayner et al., 1996; S. C. Sereno, 1992), which is at odds with O'Regan's argument. There is also evidence that contextual variables influence whether a reader will make an additional fixation on a word. Balota et al. (1985) showed that readers were less likely to have their next fixation remain in the currently fixated word if it was predictable in the sentence context.

\section{What Causes Word Skipping?}

Contextual constraint has a large effect on skipping: Words that are highly constrained by the preceding context are skipped more frequently than words that are not constrained (Balota et al., 1985; Binder, Pollatsek, \& Rayner, in press; S. F. Ehrlich \& Rayner, 1981; Rayner \& Well, 1996; Schustack, Ehrlich, \& Rayner, 1987; Vitu, 1991c). There is also some evidence to suggest that high-frequency words are skipped more than lowfrequency words, particularly if the eyes are close to the target word on the fixation prior to skipping (Radach \& Kempe, 1993; Rayner et al., 1996). However, the most important variable in word skipping is word length: Short words are much more likely to be skipped than long words (Brysbaert \& Vitu, 1998; Rayner, 1979a). These facts suggest that when readers skip a word, they identify it on the prior fixation (Rayner \& Duffy, 1988). Indeed, when words are skipped, the fixation duration prior to the skip is inflated (Hogaboam, 1983; Pollatsek et al., 1986).

In summary, the data suggest that, in addition to word-length information, textual variables appear to influence where the readers' eyes move to next. Word length seems to be the primary cue used in the normal sequence in which the word to the right of fixation has not been identified (so that the reader fixates on word $n$ and then moves to word $n+1$ ). Textual variables appear to influence the decision of whether to make the next fixation on the currently fixated word, to skip the next word, or to make a regression back to some earlier part in the text.

\section{When to Move the Eyes}

The ease or difficulty associated with processing a fixated word influences when the eyes move; much of this research is reviewed in a later section (see Rayner \& Sereno, 1994a, for a complete summary). One variable that obviously influences gaze duration on a word is word length: As word length increases, gaze duration increases (Just \& Carpenter, 1980; Rayner et al., 1996). Much of this effect is due to the fact that as words get longer, the probability that readers refixate it before moving on increases. This section, however, focuses on two variables that, when word length is controlled, strongly influence fixation time on a word: word frequency and contextual constraint.

Many studies have demonstrated that readers look longer ( first 
fixation, gaze, and single fixation duration) at low-frequency words than at high-frequency words (Altarriba, Kroll, Sholl, \& Rayner, 1996; Henderson \& Ferreira, 1990, 1993; Hyönä \& Olson, 1995; Inhoff \& Rayner, 1986; Just \& Carpenter, 1980; Kennison \& Clifton, 1995; Raney \& Rayner, 1995; Rayner, 1977; Rayner \& Duffy, 1986; Rayner \& Fischer, 1996; Rayner \& Raney, 1996; Rayner et al., 1996; Rayner, Fischer, et al., 1998; S. C. Sereno, 1992; Vitu, 1991c). There are three additional points with respect to this finding. First, there is a spillover effect from fixating low-frequency words as fixation time on the next word is inflated (Rayner \& Duffy, 1986). Second, although the duration of fixation $n$ is influenced by word frequency, the duration of fixation $n-1$ (when the target word is to the right of fixation) is not (Carpenter \& Just, 1983; Henderson \& Ferreira, 1993; Rayner, Fischer, et al., 1998). Third, highfrequency words are skipped more than low-frequency words when words are 6 letters or less (O'Regan, 1979; Rayner et al., 1996).

S. F. Ehrlich and Rayner (1981) found that words that are constrained by preceding context are fixated for less time and skipped more often than unconstrained words ( see also Altarriba et al., 1996; Balota et al., 1985; Inhoff, 1984; Rayner \& Well, 1996; Schustack et al., 1987; Zola, 1984). Hyönä (1993) subsequently found that when high-constraint target words were not as constrained as those used by S. F. Ehrlich and Rayner, ${ }^{12}$ there were no significant differences in either fixation time or skipping rates. However, more recently, Rayner and Well (1996) found that readers fixated low-constraint target words longer than they fixated high-or medium-constraint target words and that readers skipped high-constraint target words more than they did either medium- or low-constraint target words.

\section{Models of Eye Movement Control}

The issue of when to move the eyes is at the heart of a recent controversy concerning different types of models of eye movement control in reading. Such models can be classified into two general categories: (a) processing models, which assign lexical processing or other ongoing comprehension processes a major role in influencing when the eyes move, and (b) oculomotor models, which maintain that eye movements are mainly controlled by oculomotor factors and are only indirectly related to ongoing language processing. The first category includes a model proposed by Morrison (1984), with various modifications (Henderson \& Ferreira, 1990; Kennison \& Clifton, 1995; Pollatsek \& Rayner, 1990; S. C. Sereno, 1992), as well as one proposed by Just and Carpenter (1980). The second category includes a model proposed by O'Regan $(1990,1992)$, as well as proposals of Kowler and Anton (1987) and McConkie et al. (1989; McConkie, Kerr, et al., 1988). Because of the importance of the models of Morrison and O'Regan in generating research on the issue of eye movement control, each is discussed.

According to Morrison's model, which was influenced by Becker and Jürgens (1979) and McConkie (1979), at the beginning of each eye fixation, eye location and visual attention are oriented at the same location: the foveal word (word $n$ ). After foveal word processing has reached a criterion level (such as lexical access), attention shifts to the parafoveal word (word $n$ +1 ) during the fixation. This shift of attention allows processing of word $n+1$ to begin and signals the eye movement system to prepare a motor program for an eye movement to the newly attended location. Once the motor program is completed, it is executed, and the eyes then move to the new word. Because there is a lag between the attention shift and the eye movement due to programming latency, information continues to accumulate from the parafoveal word before it is directly fixated. If the parafoveal word is identified quickly, attention shifts again to the word beyond the parafoveal word (word $n+2$ ) before the eye movement is fully programmed. In this case, the eyes saccade to word $n+2$, skipping word $n+1$. Usually, there is a cost in modifying the motor program, and, as noted in a prior section, the duration of the fixation prior to a skip is consequently inflated (Hogaboam, 1983; Pollatsek et al., 1986). If the motor program is too far advanced, however, there will be either (a) a short fixation on word $n+1$ followed by a longer fixation on word $n+2$ or (b) a fixation located at an intermediate position between words $n+1$ and $n+2$. The model thus can explain some rather puzzling aspects of eye movement behavior in reading such as (a) very short fixations in text (given that saccadic latency in simple oculomotor tasks is typically on the order of 175-200 ms) and (b) unusual landing sites (e.g., the spaces between words). Although Morrison did not discuss the issue of where in a word readers fixate, most processing models assume that low-level cues such as word length influence where readers fixate (Rayner et al., 1996).

One problem in Morrison's original model was that there is no explanation for why words are sometimes refixated. That is, if lexical access is the trigger for attentional shifts (and hence eye movements), words should never be refixated. Some modifications of the model (Henderson \& Ferreira, 1990; S. C. Sereno, 1992) incorporated a deadline for programming an eye movement: If lexical processing has not reached a criterion level by this deadline, attention does not shift from the current word and it may be refixated. Another account of why words are refixated proposed by Pollatsek and Rayner (1990) did not involve any type of deadline. Rather, they suggested that the signal to stay on a word may be related to a decision that something does not fully compute; for example, the word that has been accessed may not fit into the syntactic or semantic structure of the sentence being constructed. These models do not deny that lexical effects can occur in the first fixation of refixated words; they merely state that lexical processing may not be complete enough to warrant an attentional shift to the following word.

According to O'Regan's strategy-tactics model (1990, 1992 ), the eyes' initial landing position in a word largely determines how long to remain fixated and where the following fixation is made. He proposed that readers adopt a global "strategy" (e.g., careful or risky reading) that coarsely influences fixations and saccades. He also proposed that readers implement local, within-word "tactics" that are based on lower level, nonlexical

\footnotetext{
${ }^{12}$ In S. F. Ehrlich and Rayner's (1981) study, the high-constraint target words were identified $80-90 \%$ of the time in a cloze task, and the low-constraint words were identified less than $10 \%$ of the time. In Hyönä's (1993) study, the high-constraint words were identified $65 \%$ of the time, and the low-constraint words were identified $32 \%$ of the time in a cloze task.
} 
information available early in a fixation. If the eyes land in a region of a word that is optimal (near the word's middle), there will be a single fixation. However, if they land in a nonoptimal position, a refixation will generally occur. Fixation durations according to this scheme are mainly determined by oculomotor constraints. The probability that a word is refixated does not depend on its lexical status but on lower level visual factors such as the landing position in that word. Linguistic factors influence the duration of a single long fixation $(300 \mathrm{~ms}$ or longer; O'Regan, 1992) but only the second of two fixations in a refixated word.

A problem with O'Regan's model is that the optimal viewing position effect was originally obtained in tasks in which words were presented in isolation (Nazir, Heller, \& Sussmann, 1992; O'Regan \& Jacobs, 1992; O'Regan \& Lévy-Schoen, 1987; O'Regan et al., 1984). Normal reading, however, involves more than recognizing individual words. As noted previously, although the refixation effect (i.e., readers are more likely to refixate a word if the initial fixation is away from the optimal viewing position) was obtained in reading, the processing-cost effect (i.e., a cost of $20 \mathrm{~ms}$ per character that the fixation is away from the optimal viewing position) was greatly attenuated or absent (Vitu, 1991c; Vitu et al., 1990).

A number of studies have tried to discriminate between these models. For example, Vitu and O'Regan (1995; see also Schroyens et al., in press) presented data that they argued are inconsistent with the oculomotor deadline proposed by Henderson and Ferreira (1990); specifically, they found that the duration of a single fixation is longer than the first of two fixations (see also Rayner et al., 1996; Schroyens et al., in press). Models with an oculomotor deadline should predict just the opposite, because when a second fixation on a word takes place, the deadline would be approached by the first fixation. On the other hand, Rayner et al. (1996) argued that data they obtained were inconsistent with the oculomotor type of model; specifically, they found that when single fixations were made on a word, there was a frequency effect independent of where the reader fixated. In contrast, Reilly and O'Regan (1998) reported a number of simulations that led them to argue that the oculomotor type of model can better explain the details of where readers initially fixate in words. Although Reilly and O'Regan's analyses are quite valuable, because they demonstrate the value of using simulations to account for data patterns, their conclusion is not too surprising given that where the eyes land was never a central issue of processing models. Finally, Vitu, O'Regan, Inhoff, and Topolski (1995) examined eye movements when participants scanned transformed text (in which each letter was replaced with a $z$ ) and found that the eye movements were globally quite similar to when the same participants read text. Thus, they argued that the similarity of the pattern suggested that predetermined oculomotor strategies were an important factor in eye movement control in reading. However, Rayner and Fischer (1996) showed that there are important differences between the two situations and that eye movements in reading are influenced by immediate processing demands.

Although there are extant data supporting both types of model, Rayner et al. (1996) argued that the evidence is more consistent with the processing type of model than the oculomotor type of model. More critically, they argued that neither type of model is sufficiently precise for comprehensive testing. Thus, they suggested that future instantiations of models of eye movement control must be more explicit, perhaps through the use of mathematical or computational modeling. One such model was proposed by Just and Carpenter (1980) and a number have appeared recently (see Legge, Klitz, \& Tjan, 1997; Reichle, Pollatsek, Fisher, \& Rayner, 1998; Reilly, 1993; Reilly \& O'Regan, 1998; Suppes, 1990, 1994). These models provide the hope of more explicit testing of underlying claims related to eye movement control.

While Suppes's stochastic model $(1990,1994)$ has the virtue of mathematical precision, it cannot account for many effects that are known to influence fixation durations. Mr. Chips, the model proposed by Legge et al. (1997), is based to some extent on work done on the impact of low vision on reading (Legge, Rubin, Pelli, \& Schleske, 1985). As such, it is an interesting attempt to generalize from low-vision reading to normal reading. However, many of the premises made by the model seem unrealistic in terms of real-time processing events that occur in normal reading. The Reader model of Just and Carpenter (1980; see also Thibadeau, Just, \& Carpenter, 1982) attempted to account for reading processes from individual eye fixations to comprehension processes. Although it was intended as a comprehensive model of reading, very few predictions can be derived from the model that are relevant for eye movement control. The $E-Z$ Reader model (Reichle et al., 1998; see also Rayner, Reichle, \& Pollatsek, 1998), on the other hand, is a formal instantiation of Morrison's model (but with a number of modifications ${ }^{13}$ ) and applies directly to eye movement control. Reichle et al. compared actual eye movement data with simulations from the model, and the fit between the model and data was very good. Furthermore, the model has the virtue that it accounts for many findings discussed in prior sections (e.g., preview benefits, frequency effects, spillover effects, constraint effects on skipping behavior) while also making explicit predictions. ${ }^{14}$

In summary, there is controversy surrounding the issue of eye movement control, but some findings and conclusions seem incontroversial. Specifically, where readers look next is related to the length of upcoming words (and where in the current word the reader is fixated), and how long they fixate on a word is related to the ease or difficulty associated with processing that word. It is hoped that more precision will be obtained on these issues as models such as that of Reichle et al. (1998) appear.

\section{Using Eye Movements to Study Language Processing in Reading}

Considerable data have been collected that demonstrate that eye movements are intimately related to the moment-to-moment

\footnotetext{
${ }^{13}$ For example, in the E-Z Reader model, a familiarity check that precedes lexical access serves as the signal for the initiation of an eye movement, and completion of lexical access is the signal for a shift of covert attention.

${ }^{14}$ As an example, the model predicted that when a word is skipped, the duration of the fixation prior to the skip and the duration of the fixation following the skip should both be inflated. Whereas the former effect had been previously noted (Hogaboam, 1983; Pollatsek et al., 1986), the latter had not. However, examination of data revealed such an effect.
} 
Table 2

Variables and Specific Studies Influencing Eye Movements in Reading

\begin{tabular}{|c|c|}
\hline Variable & Study \\
\hline $\begin{array}{l}\text { Semantic relationships between } \\
\text { words }\end{array}$ & Carroll \& Slowiaczek, 1986; Morris, 1994; S. C. Sereno, 1995; S. C. Sereno \& Rayner, 1992 \\
\hline Repetition effects & Hyönä \& Niemi, 1990; Inhoff et al., 1993; Raney \& Rayner, 1995; Rayner, Raney, \& Pollatsek, 1995 \\
\hline Morphemic units & $\begin{array}{l}\text { Beauvillain, 1996; Holmes \& O'Regan, 1987, 1992; Hyönă \& Pollatsek, in press; Inhoff, Briihl, \& } \\
\text { Schwartz, 1996; Lima, } 1987\end{array}$ \\
\hline Anaphora and coreference & $\begin{array}{l}\text { Albrecht \& Clifton, 1998; Blanchard, 1987; Duffy \& Rayner, 1990; K. Ehrlich, 1983; K. Ehrlich \& } \\
\text { Rayner, 1983; Garrod, Freudenthal, \& Boyle, 1994; Garrod, O'Brien, Morris, \& Rayner, 1990; } \\
\text { Kerr \& Underwood, 1984; Kennison \& Gordon, 1998; O'Brien, Raney, Albrecht, \& Rayner, 1997; } \\
\text { O'Brien, Shank, Myers, \& Rayner, 1988; Paterson, Sanford, Moxey, \& Dawydiak, 1998; Vonk, } \\
\text { 1984, } 1985\end{array}$ \\
\hline Lexical ambiguity & $\begin{array}{l}\text { Binder \& Morris, 1995; Binder \& Rayner, 1998; Dopkins, Morris, \& Rayner, 1992; Duffy, Morris, \& } \\
\text { Rayner, 1988; Frazier \& Rayner, 1990; Pacht \& Rayner, 1993; Rayner \& Duffy, 1986; Rayner \& } \\
\text { Frazier, 1989; Rayner, Pacht, \& Duffy, 1994; S. C. Sereno, 1995; S. C. Sereno, Pacht, \& Rayner, } \\
1992\end{array}$ \\
\hline Phonological ambiguity & $\begin{array}{l}\text { Carpenter \& Daneman, 1981; Daneman \& Reingold, 1993; Daneman, Reingold, \& Davidson, 1995; } \\
\text { Folk, 1998; Folk \& Morris, 1995; Inhoff \& Topolski, 1994; Jared, Levy, \& Rayner, in press; }\end{array}$ \\
\hline . & $\begin{array}{l}\text { Lesch \& Pollatsek, 1993, 1998; Pollatsek et al., 1992; Rayner, Pollatsek, \& Binder, 1998; Rayner, } \\
\text { Sereno, Lesch, \& Pollatsek, 1995 }\end{array}$ \\
\hline $\begin{array}{l}\text { Discourse factors and stylistic } \\
\text { conventions }\end{array}$ & $\begin{array}{l}\text { Birch \& Rayner, 1997; Blanchard \& Iran-Nejad, 1987; Carrithers \& Bever, 1984; Dee-Lucas, Just, } \\
\text { Carpenter, \& Daneman, 1982; Deutsch, 1998; Fletcher, 1990, 1991, 1993; Frisson \& Pickering, } \\
\text { 1998; Grabe, Antes, Kahn, \& Kristjanson, 1991; Grabe, Antes, Thorson, \& Kahn, 1987; Hyönä, } \\
\text { 1995b; Hyönä \& Hujanen, 1997; Hyönä \& Jarvella, 1993; Inhoff, 1985; Kennedy \& Pidcock, 1981; } \\
\text { Morris \& Folk, 1998; Rothkopf \& Billington, 1979; Scinto, 1978; Vauras, Hyönä, \& Niemi, } 1992\end{array}$ \\
\hline Syntactic disambiguation & $\begin{array}{l}\text { Adams, Clifton, \& Mitchell, 1998; Altmann, 1994; Altmann, Garnham, \& Dennis, 1992; Altmann, } \\
\text { Garnham, \& Henstra, 1994; Altmann, van Nice, Garnham, \& Henstra, 1998; Britt, Perfetti, } \\
\text { Garrod, \& Rayner, 1992; Brysbaert \& Mitchell, 1996; Clifton, 1992, 1993; Clifton, Speer, \& } \\
\text { Abney, 1991; Ferreira \& Clifton, 1986; Ferreira \& Henderson, 1990, 1993; Ferreira \& McClure, } \\
\text { 1997; Frazier \& Clifton, 1998; Frazier \& Rayner, 1982, 1987, 1988; Frenck-Mestre \& Pynte, 1997; } \\
\text { Garnsey, Pearlmutter, Myers, \& Lotocky, 1997; Holmes \& O'Regan, 1981; Kennedy, Murray, } \\
\text { Jennings, \& Reid, 1989; Konieczny, Hemforth, Scheepers, \& Strube, 1997; Liversedge, Pickering, } \\
\text { Branigan, \& van Gompel, 1998; Mazuka, Itoh, \& Kondo, 1997; Murray \& Liversedge, 1994; Ni, } \\
\text { Crain, \& Shankweiler, 1996; Ni, Fodor, Crain, \& Shankweiler, 1998; Paterson, Liversedge, \& } \\
\text { Underwood, in press; Pickering \& Traxler, 1998; Rayner, Carlson, \& Frazier, 1983; Rayner \& } \\
\text { Frazier, 1987; Rayner, Garrod, \& Perfetti, 1992; Rayner \& Sereno, 1994b, 1994c; Schmauder, } \\
\text { 1991; Schmauder \& Egan, 1998; Speer \& Clifton, 1998; Traxler, Bybee, \& Pickering, 1997; } \\
\text { Traxler \& Pickering, 1996a, 1996b; Trueswell, Tanenhaus, \& Garnsey, 1994; Trueswell, } \\
\text { Tanenhaus, \& Kello, 1993; Zagar, Pynte, \& Rativeau, 1997 }\end{array}$ \\
\hline
\end{tabular}

cognitive processing activities of readers. Two variables, word frequency and contextual constraint, were discussed in a prior section. Table 2 lists a number of other variables that influence fixation time on a word or the pattern of eye movements. Briefly, with respect to these variables, the findings are as follows. First, words that are semantically related and in close proximity to each other produce priming effects. Thus, the word king in the same clause results in shorter fixation time on queen than if an unrelated word is present (Carroll \& Slowiaczek, 1986; Morris, 1994). Second, when text is reread, fixation duration decreases (Hyönä \& Niemi, 1990; Inhoff et al., 1993); when high- and low-frequency words are encountered a number of times within a passage, fixation times on the words decrease, with the effect being more pronounced for low-frequency words ${ }^{15}$ (Rayner, Raney, \& Pollatsek, 1995). Third, readers look longer at prefixed words than pseudoprefixed words (Lima, 1987) and longer at morphemes in long words that are more informative with respect to the overall meaning of the word (Hyönä \& Pollatsek, in press). Fourth, fixation time in the region of a pronoun varies as a function of how easy it is to make the link between the pronoun and its antecedent (K. Ehrlich \& Rayner, 1983), and words that invite the reader to make an elaborative inference are fixated for less time than when such an inference is not made. For example, O'Brien, Shank, Myers, and Rayner (1988) found that when the phrase stabbed her with his weapon was read early in a passage, readers subsequently fixated no longer on the target word knife than when the earlier phrase read stabbed her with his knife; in the weapon case, the reader presumably inferred that the weapon was a knife. By contrast, when the original phrase read assaulted her with his weapon, readers fixated longer on the subsequent occurrence of knife. Fifth, fixation times on lexically ambiguous words are modulated by the characteristics of the word and the prior context. The basic finding is that when the preceding context is neutral

\footnotetext{
${ }^{15}$ A curious fact is that during the first and second era of eye movement recording, researchers did not report effects of linguistic variables. For the most part, such effects were not examined, but they may not have been noticed because the tendency was to record eye movements while readers read passages of text. In such passages, words would often be repeated throughout the passage. Because the repetition effect observed by Inhoff et al. (1993) and Rayner et al. (1995) results in pretty much eliminating the word frequency effect, effects of word frequency would not be apparent.
} 
(so that it does not bias either meaning of the word), readers look no longer at a biased ambiguous word (one with a highly dominant meaning) than a control word (matched on length and frequency); however, they do look longer at a balanced ambiguous word (with two equally likely meanings) than at a control word. On the other hand, when the context biases one meaning, readers look no longer at a balanced word than the control; however, if the context biases the subordinate meaning of the word, they look longer at a biased word than at a control word (Duffy, Morris, \& Rayner, 1988). Sixth, when a word is replaced by a homophone, readers look at it longer (Daneman \& Reingold, 1993) unless the context is highly constraining of the correct word (Rayner, Pollatsek, \& Binder, 1998). Seventh, stylistic conventions such as focus and dovetailing (the final content word of a sentence and the first content word of the next one have the same referent) influence eye movements (Birch \& Rayner, 1997; Kennedy \& Pidcock, 1981). Finally, if an incorrect interpretation of a syntactically ambiguous phrase is made, fixation times on the disambiguating word increase or readers make an immediate regression (Frazier \& Rayner, 1982).

The above findings raise some important issues with respect to eye movements and reading. First, what exactly controls fixation time on a word? Some of the effects listed above are undoubtedly related to postlexical processing, so is the decision to move off of a word based on lexical or postlexical processing? The answer to this question is not immediately obvious. It could be argued that the following variables influence lexical access: word frequency, contextual constraint, semantic relationships between words, lexical ambiguity, and phonological ambiguity. However, it seems less plausible that syntactic disambiguation effects are due to lexical access processes: When readers encounter a disambiguating word, the lengthened fixation time must surely be due to postaccess integration processes. Likewise, coreference and anaphoric processing consists of integration processing. The best answer to the question (Carroll \& Slowiaczek, 1987; Hyönä, 1995b; Pollatsek, 1993; Rayner \& Morris, 1990) is that lexical access (or familiarity check; Reichle et al., 1998) is the trigger to program a new eye movement but that other higher level (postaccess) processes intervene when something does not compute. Thus, fixation times primarily reflect lexical processing, but postaccess integration effects can have an effect as well.

A second general question concerns whether effects of experimental manipulations on fixation time measures are produced by similar effects in all relevant cases or by combinations of unequal effects in subsets of the data. McConkie (see McConkie, Reddix, \& Zola, 1992; McConkie, Underwood, Wolverton, \& Zola, 1988; McConkie, Zola, \& Wolverton, 1985) has discussed the theoretical and methodological implications of this "frequency-of-effects" issue and has shown that when $20 \%$ of the fixations are affected by a particular manipulation with the size of the effect being $100 \mathrm{~ms}$, even though the rest of the fixations are not affected there will still be a 20 -ms effect. On the basis of such arguments, some researchers (Rayner, 1995; Rayner \& Fischer, 1996) have shown frequency distributions for the effect being investigated to demonstrate that the distribution shifted and was not due to a subset of the data.

A final question that is relevant with respect to the findings discussed in this section concerns the extent to which eye move- ment data can be mimicked by other laboratory tasks. One great virtue of eye movement data is that readers are engaged in the task of normal reading and can proceed at their own rate. Many years ago, Tinker (1939) demonstrated that reading rate and comprehension were the same when readers read in an eye movement laboratory (with their heads fixed) and when they read in an easy chair. Given the cost and effort that is required to obtain eye movement data, many researchers have opted for tasks that are thought to simulate eye movement data. The two primary techniques that have been used are rapid serial visual presentation (RSVP) of text and self-paced reading. In the RSVP task, words are presented at a set rate (such as every $100-200 \mathrm{~ms}$ ) in the same spatial location. Comparisons of this task to normal reading have typically revealed that with short sentences, results that are typical of normal reading can be obtained. However, when longer passages are presented, the processing system quickly gets overloaded, and comprehension breaks down (see Masson, 1983). With self-paced reading, text is presented a word or two at a time and readers advance the text by pushing a button. There are a number of variations of this technique: Some allow readers to look back in text (cumulative methods), some do not (so that only 1-2 words are on the screen at a time); and in some, words are presented sequentially in the same location, whereas in others, words are presented spatially as in normal text. There are clear trade-offs associated with these techniques. One virtue when only 1 word is presented is that a processing-time measure is associated with each word in the text (i.e., words cannot be skipped). However, the most natural method, the self-paced cumulative moving window, involves cumulative presentation of words so that, if a reader looks back in the text before pushing the button, there is no way of knowing this. For this reason, this technique is seldom used. The altemative, self-paced noncumulative moving window, has the problem that readers are unable to reread words they did not understand (or misanalyzed).

A general problem with all of the variations of the self-paced reading technique is that reading rate is about half as fast as it is normally. The fact that readers are slowed down by the demands of the task (because the reaction time of the eyes is faster than the reaction time of the fingers) suggests that some caution has to be exerted in generalizing from these tasks to normal reading (Rayner, Sereno, et al., 1989). There have been many comparisons of RSVP and self-paced reading tasks to normal reading (Juola, Ward, \& McNamara, 1982; Just, Carpenter, \& Woolley, 1982; Kennedy \& Murray, 1984; Rubin \& Turano, 1992). In many cases, similar results have been obtained, but this is not always the case (Just, Carpenter, \& Woolley, 1982; Kennedy \& Murray, 1984; Magliano, Graesser, Eymard, Haberlandt, \& Gholson, 1993). Researchers interested in the topic of syntactic ambiguity and parsing have been particularly keen to show that similar results are obtained when self-paced reading and eye movement data are compared (see Ferreira \& Clifton, 1986; Garnsey, Pearlmutter, Myers, \& Lotocky, 1997; Kennedy \& Murray, 1984; Trueswell, Tanenhaus, \& Kello, 1993); indeed many results are quite similar across the two situations. Perhaps it is not surprising that, when dealing with syntactic ambiguity where readers are "garden-pathed" and have difficulty getting the appropriate meaning, self-paced reading and eye movement data are quite similar because processing has 
been disrupted and readers must use some type of problemsolving strategy to compute the correct meaning. Given that they are going slowly in both situations, the results might well be fairly comparable. Even if this is the case, caution is warranted in generalizing from self-paced reading to normal reading because of the depressed reading rate.

How well do standard laboratory tasks like naming and lexical decision compare with eye movement data? Everatt and Underwood (1994) found little correlation between eye fixation times and lexical decision times. However, Schilling, Rayner, and Chumbley (1998) compared naming and lexical decision times for words presented in isolation to fixation times on the same words inserted in neutral sentence contexts and found reasonably good correlations between the measures. The size of the word-frequency effect (i.e., the difference in reaction times for high- and low-frequency words) was fairly equivalent for naming and gaze duration (with the size of the effect a bit smaller for first fixation and single fixation duration). The size of the frequency effect was exaggerated in the lexical decision times compared with the eye fixation times. These results suggest that researchers can have some confidence that results obtained with standard naming and lexical decision tasks generalize to word recognition processes while reading. ${ }^{16}$

Another procedure that has been adopted recently is to use the same stimulus materials across different tasks to obtain converging evidence on a topic of interest (see Altarriba et al., 1996; Inhoff, Briihl, et al., 1996; O'Brien, Raney, Albrecht, \& Rayner, 1997; Schustack et al., 1987). Important information can be gleaned both when the results are consistent (e.g., the results are generalizable across tasks) and when they are inconsistent. In the latter case, differences across tasks can enlighten our understanding not only of the specific tasks but of the processes involved. Recently, studies (Raney \& Rayner, 1993; S. C. Sereno, Rayner, \& Posner, 1998) have been reported with eventrelated potential data in conjunction with eye movement data, which makes it possible to address issues that would be otherwise difficult to examine. For example, S. C. Sereno et al. (1998) examined the time line of lexical access and saccade programming.

Finally, a technique called fast priming (S. C. Sereno \& Rayner, 1992) combines priming techniques with eye movement paradigms. In this technique, a random string of letters initially occupies a target location in a sentence. When the reader's eye movement crosses a boundary location, the random letter string is replaced by a prime word that is presented briefly (30-40 $\mathrm{ms}$ ) at the onset of the fixation and then changes to the target word. Studies using this technique have examined semantic priming (S. C. Sereno, 1995; S. C. Sereno \& Rayner, 1992), phonological priming (Lee, Binder, Kim, Pollatsek, \& Rayner, in press; Rayner, Sereno, Lesch, \& Pollatsek, 1995), and orthographic priming (Lee et al., in press; Rayner, Sereno, et al., 1995).

\section{Eye Movements When Reading and Looking at Pictures}

Carroll, Young, and Guertin (1992) found that when viewers looked at cartoons, mean fixation durations on the cartoon were longer than on the caption. There was not a great deal of consistency in where viewers looked first. They did not move back and forth repeatedly between cartoon elements, and the picture frequently was not given a full inspection until the caption was read. Similar results were obtained when complex mechanical diagrams in text (Hegarty, 1992a, 1992b) and subtitled TV programs (d'Ydewalle \& Gielen, 1992) were used.

\section{Eye Movements and Individual Differences in Reading}

There are well-known individual differences in eye movement measures as a function of reading skill: Fast readers make shorter fixations, longer saccades, and fewer regressions than slow readers (Everatt, Bradshaw, \& Hibbard, 1998; Everatt \& Underwood, 1994; Rayner, 1978b; G. Underwood, Hubbard, \& Wilkinson, 1990). Likewise, bilingual readers make shorter fixations, longer saccades, and fewer regressions in their dominant language (Altarriba et al., 1996; Chincotta, Hyönä, \& Underwood, 1997), and children who stutter make more fixations and regressions than nonstutterers (Brutten, Bakker, Janssen, \& VanderMeulen, 1984). In characterizing the eye movement patterns of dyslexic readers, Olson, Kliegl, Davidson, and Foltz (1985) categorized such readers as plodders and explorers; plodders made relatively short forward saccades and very few regressions, whereas explorers showed more frequent word skipping, longer forward saccades, and more regressions. It is likely that skilled readers can be divided into similar styles (Rothkopf, 1978). Table 3 shows eye movement characteristics of 10 skilled readers with good comprehension. It is clear that there is a considerable amount of difference been them. In the remainder of this section, studies dealing with speedreading, developmental changes in eye movements, and eye movements of dyslexic readers are discussed.

\section{Speedreading}

Unfortunately, there is very little good data on the characteristics of the eye movements of speedreaders. Rayner and Pollatsek (1989) reviewed some early studies but pointed out that the studies were disappointing because of inadequate control conditions or because comprehension tests were not taken. The only good recent report of the characteristics of speedreaders' eye movements was provided by Just, Carpenter, and Masson (1982; also reported in Just \& Carpenter, 1987). They monitored the eye movements of speedreaders (reading at approximately $600-$ 700 words per minute [wpm]) and normal readers (reading

\footnotetext{
${ }^{16}$ Not every variable that affects isolated word recognition influences eye movements in reading. For example, Rayner and Duffy (1986) found no effect of verb complexity on fixation times; Inhoff, Lima, and Carroll (1984) found little effect of whether or not a literal or metaphoric interpretation was associated with a target word (though regression frequency was affected); Perea and Pollatsek (1998) found effects of neighborhood frequency on only spillover fixations (see Pollatsek, Perea, and Binder, in press, for earlier affects; and see Grainger, O'Regan, Jacobs, \& Sequi, 1992, for how initial fixation location and neighborhood frequency interact for isolated words); and Inhoff and Topolski (1994) found that the effects of orthographic regularity were rather transient in the eye movement record. However, S. C. Sereno, Rayner, and Posner (1998) found a regularity effect for low-frequency words, whereas, consistent with word recognition studies, there was no effect for highfrequency words.
} 
Table 3

Mean Fixation Duration, Mean Saccade Length, Percentage Regressions, and Words per Minute (WPM) for 10 Skilled Readers

\begin{tabular}{lcccc}
\hline Reader & $\begin{array}{c}\text { Fixation } \\
\text { duration }\end{array}$ & $\begin{array}{c}\text { Saccade } \\
\text { length }\end{array}$ & $\begin{array}{c}\text { Regressions } \\
(\%)\end{array}$ & WPM \\
\hline KB & 195 & 9.0 & 6 & 378 \\
JC & 227 & 7.6 & 12 & 251 \\
AK & 190 & 8.6 & 11 & 348 \\
TP & 196 & 9.5 & 15 & 382 \\
TT & 255 & 7.7 & 19 & 244 \\
GT & 206 & 7.9 & 4 & 335 \\
GB & 205 & 8.5 & 6 & 347 \\
BB & 247 & 6.7 & 1 & 257 \\
LC & 193 & 8.3 & 20 & 314 \\
JJ & 241 & 7.2 & 14 & 230 \\
$M$ & 216 & 8.1 & 11 & 308 \\
\hline
\end{tabular}

Note. Fixation duration is in milliseconds, and saccade length is in character spaces ( 4 characters $=1^{\circ}$ of visual angle). The text was fairly easy reading. If the text were more difficult, for each reader, the mean fixation duration would likely increase, the mean saccade length would likely decrease, the percentage of regressions would likely increase, and the reading rate would decrease.

From "Eye Movements in Reading and Information Processing"' by $\mathbf{K}$. Rayner, 1978b, Psychological Bulletin, 85, p. 627. Copyright 1978 by the American Psychological Association.

around $250 \mathrm{wpm}$ ). In addition, the normal readers were asked to "skim" the text to produce rates around 600-700 wpm. When tested after reading, the speedreaders did as well as the normal readers (when reading at their normal speed) on general comprehension questions or questions about the gist of the passage. However, when tested about the details of the text, speedreaders could not answer questions if they had not fixated on the region where the answer was located. The normal readers, whose fixations were much denser than the speedreaders, were able to answer the detail questions relatively well. When the normal readers were asked to skim the text, both their eye movement patterns and comprehension measures were quite similar to those of the speedreaders. In general, the results of research on the characteristics of speedreaders' eye movements are consistent with the idea that they are skimming the text and not really reading every word (see Rayner \& Pollatsek, 1989, for more details).

\section{Developmental Changes in Eye Movements}

For over 70 years (Buswell, 1922), it has been known that there are developmental trends in eye movements during reading: As reading skill increases, fixation duration decreases, saccade length increases, the number of fixations decreases, and the frequency of regressions decreases. Table 4 shows summary data from Buswell (1922), S. E. Taylor (1965), Rayner (1985b), and McConkie et al. (1991) on different measures. Although eye movements were recorded in very different ways, the trends in the data are remarkably consistent.

McConkie et al. (1991) examined children's eye movement behavior and found that they tended to have more variability in their eye movement indices than adults. However, they also found that children in their 1st year of reading show the same landing position patterns as adults; like adults, they send their eyes to the middle of a word. McConkie et al. (1991) also concluded that one of the primary differences between the eye movements of children and adults is the frequency with which words are refixated before the reader moves to another word; adults refixated 5 letter words $15 \%$ of the time, whereas firstgrade children refixated 5 letter words $57 \%$ of the time. As noted earlier, Rayner (1986) found that the perceptual span of beginning readers was smaller than skilled readers and that the span was asymmetric for beginning readers. Apparently, 1 year of reading is sufficient for the development of the asymmetry of the span. Finally, the effect of aging on eye movements in reading is not completely clear. Solan, Feldman, and Tujak (1995) found that elderly adults read more slowly and made more fixations (and regressions) than younger readers. However, they also found that reading efficiency training greatly improved performance. Although Solan et al. did not examine fixation durations, it is highly likely that they increase, because saccade latency in elderly adults is longer than younger adults (Abel et al., 1983).

\section{Eye Movements, Poor Readers, and Dyslexia}

Poor readers and dyslexic readers, like beginning readers, make longer fixations, shorter saccades, more fixations, and more regressions than normal readers. These facts have been known for some time and have been reinforced by many recent studies (Adler-Grinberg \& Stark, 1978; Eden, Stein, Wood, \& Wood, 1994; Elterman, Abel, Daroff, Dell'Osso, \& Bornstein, 1980; Lefton, Nagle, Johnson, \& Fisher, 1979: Martos \& Vila, 1990). Lefton et al. (1979) found that normal developmental gains made by children (wherein fixation duration decreases, saccade length increases, and the frequency of regressions decreases) are not shown by dyslexic readers.

No one would dispute that the eye movements of dyslexic readers are different from normal readers. One critical question that reemerged recently concerns the extent to which eye movements per se represent the cause of reading problems. Certainly, people with low vision (Bowers \& Reid, 1997; Bullimore \& Bailey, 1995; Legge, Rubin, Pelli, \& Schleske, 1985), oculomotor disturbances such as saccade intrusions (Ciuffreda, Kenyon, \& Stark, 1983), or congenital jerk nystagmus (Ciuffreda, 1979) have difficulty reading (see Ciuffreda, 1994, and Ciuffreda \& Tannen, 1995, for more extensive discussion of the eye movements of patients with oculomotor disturbances ). The more critical issue is whether or not reading disability in the absence of obvious oculomotor problems can be attributed to faulty eye movements. If eye movements were a causative factor in reading disability, then the problem could easily be diagnosed with a simple eye movement test, and oculomotor training would result in improved reading. Although there have been some demonstrations that oculomotor training improves reading performance (see Solan, 1985), Tinker (1946, 1958) and Rayner (1985b) argued quite strongly that eye movements were generally not a cause of reading disability but were a reflection of other underlying problems.

This issue was brought back into the spotlight by Pavlidis $(1978,1981,1983,1985,1991)$. He reported experiments in 
Table 4

Developmental Characteristics of Eye Movements During Reading

\begin{tabular}{|c|c|c|c|c|c|c|c|}
\hline \multirow[b]{2}{*}{ Article and characteristic } & \multicolumn{6}{|c|}{ Grade level } & \multirow[b]{2}{*}{ Adult } \\
\hline & 1 & 2 & 3 & 4 & 5 & 6 & \\
\hline \multicolumn{8}{|l|}{ S. E. Taylor (1965) } \\
\hline Fixation duration (ms) & 330 & 300 & 280 & 270 & 270 & 270 & 240 \\
\hline Fixations per 100 words & 224 & 174 & 155 & 139 & 129 & 120 & 90 \\
\hline Frequency of regressions (\%) & 23 & 23 & 22 & 22 & 21 & 21 & 17 \\
\hline \multicolumn{8}{|l|}{ Buswell (1922) } \\
\hline Fixation duration (ms) & 432 & 364 & 316 & 268 & 252 & 236 & 252 \\
\hline Fixations per 100 words & 182 & 126 & 113 & 92 & 87 & 87 & 75 \\
\hline Frequency of regressions (\%) & 26 & 21 & 20 & 19 & 20 & 21 & 8 \\
\hline \multicolumn{8}{|l|}{ Rayner (1985b) } \\
\hline Fixation duration (ms) & - & 290 & - & 276 & - & 242 & 239 \\
\hline Fixations per 100 words & - & 165 & - & 122 & - & 110 & 92 \\
\hline Frequency of regressions (\%) & - & 27 & - & 25 & - & 24 & 9 \\
\hline \multicolumn{8}{|l|}{ McConkie et al. (1991) } \\
\hline Fixation duration (ms) & 304 & 268 & 262 & 248 & 243 & - & 200 \\
\hline Fixations per 100 words & 168 & 138 & 125 & 132 & 135 & - & 118 \\
\hline Frequency of regressions (\%) & 34 & 33 & 34 & 36 & 36 & - & 21 \\
\hline \multicolumn{8}{|l|}{$M$} \\
\hline Fixation duration (ms) & 355 & 306 & 286 & 266 & 255 & 249 & 233 \\
\hline Fixations per 100 words & 191 & 151 & 131 & 121 & 117 & 106 & 94 \\
\hline Frequency of regressions (\%) & 28 & 26 & 25 & 26 & 26 & 22 & 14 \\
\hline
\end{tabular}

Note. Dashes indicate that data were not collected.

which normal children, backward readers (whose reading disability could be accounted for by intelligence, motivation, or socioeconomic factors), and dyslexic readers followed a dot that moved across a screen. He reported that when the dot moved from left to right, dyslexic readers made many more right-toleft saccades than either the normal or backward readers. Thus, he argued that faulty eye movements or some type of central temporal ordering problem could be diagnostic of dyslexic readers (Pavlidis, 1981). Pavlidis quite rightly pointed out that examining the eye movements of dyslexic readers during reading was inconclusive because one would not be able to tell if eye movements per se or the dyslexic individual's problems with reading accounted for the differences between their eye movement characteristics and those of normal readers. The fact that erratic eye movement patterns were obtained in the absence of text thus suggested to Pavlidis that eye movements are a contributing factor to reading disability. However, a number of attempts to replicate Pavlidis's findings have failed to confirm his results (Black, Collins, DeRoach, \& Zubrick, 1984a; B. Brown, Haegerstrom-Portnoy, Adams, et al., 1983; Olson, Conners, \& Rack, 1991; Olson, Kliegl, \& Davison, 1983a; Stanley, 1994; Stanley, Smith, \& Howell, 1983a). Furthermore, other studies have failed to find differences between normal and dyslexic readers' eye movements during nonreading tasks (AdlerGrinberg \& Stark, 1978; Black, Collins, DeRoach, \& Zubrick, 1984b, 1984c; B. Brown, Haegerstrom-Portnoy, Adams, et al., 1983; B. Brown, Haegerstrom-Portnoy, Yingling, Herron, Galin, \& Marcus, 1983; Eskenazi \& Diamond, 1983; Fields, Wright, \& Newman, 1993; Olson, Conners, \& Rack, 1991; Stanley, Smith, \& Howell, 1983b).

In addition to Pavlidis, others have argued that poor eye movement control is characteristic of some dyslexic readers. Eden et al. (1994) found that dyslexic readers had worse stability of fixation on small targets than normal readers and that dyslexic readers' vergence and smooth pursuit eye movements differed from normal readers. It is not clear what to make of these findings because pursuit eye movements are not characteristic of reading. ${ }^{17}$ The finding of fixation instability was also reported by Pavlidis (1981) and Raymond, Ogden, Fagan, and Kaplan (1988). However, Raymond et al. also found that the latency and accuracy of eye movements of dyslexic children did not differ from those of normal readers. Finally, Fischer and colleagues (Biscaldi, Fischer, \& Aiple, 1994; B. Fischer, Biscaldi, \& Otto, 1993; B. Fischer \& Weber, 1990) found that some dyslexic readers exhibit more express saccades than normal readers. They argued that express saccades are related to attentional processing. Given the failures to replicate Pavlidis's work, it would be premature at this point to arrive at firm conclusions regarding the relationship between eye movements and dyslexia until these results are replicated in other labs.

It has also been suggested that dyslexic readers process less parafoveal information on each fixation than do normal readers (see Farmer \& Klein, 1995). Experiments by Rayner, Murphy, Henderson, and Pollatsek (1989) and N. R. Underwood and Zola (1986) used the moving window technique to investigate this issue. Although N.R. Underwood and Zola found little difference between good and poor readers (fifth-grade children

\footnotetext{
${ }^{17}$ Stein and colleagues (Stein, 1989, 1994; Stein, Riddell, \& Fowler 1988) have presented data to indicate that dyslexic readers have fine binocular control problems, which result in their being unable to hold their eyes steady. On the other hand, Lennerstrand, Ygge, and Rydberg (1994) found no differences between normal and dyslexic readers with respect to binocular control.
} 
reading 1-2.5 years behind their expected reading level), Rayner, Murphy, et al. (1989) found that the perceptual span was smaller for dyslexic readers than for normal readers. However, this finding does not necessarily mean that dyslexic readers process parafoveal information less effectively than do normal readers (Rayner, Pollatsek, \& Bilsky, 1995). As noted in an earlier section, normal readers obtain less parafoveal information when the fixated word is difficult to process (Henderson \& Ferreira, 1990; Rayner, 1986). Rayner (1986) showed that when children were given difficult text to read, their perceptual span got smaller. Thus, whereas the perceptual span is somewhat smaller for dyslexic readers than for normal readers, it is probably not the case that dyslexic readers process parafoveal information less effectively but rather that they have difficulty processing the fixated word.

Actually, there is some irony with respect to this issue since Geiger and Lettvin (1987) proposed that the cause of dyslexia was that dyslexic readers processed parafoveal information more effectively than normal readers. On the basis of experiments in which dyslexic and normal readers had to simultaneously identify foveal and parafoveal letters, they suggested that dyslexic readers' efficient parafoveal processing interfered with foveal processing, and this is what causes their reading problems. Indeed, Geiger, Lettvin, and Fahle (1994) argued that dyslexic readers could markedly increase their reading ability by cutting a small window in an index card and reading the material inside the window as they moved it across the text. However, there is considerable controversy regarding whether or not dyslexic readers process parafoveal information more effectively than do normal readers. Perry, Dember, Warm, and Sacks (1989) reported results that were consistent with Geiger and Lettvin (1987), but others (Goolkasian \& King, 1990; Klein, Berry, Briand, D'Entremont, \& Farmer, 1990; Slaghuis, Lovegrove, \& Freestun, 1992) were unable to replicate the findings. Finally, Rayner, Murphy, et al. (1989) described a dyslexic reader who showed characteristics that were somewhat like those of Geiger and Lettvin's (1987) participants: He could identify parafoveal words and letters better than the normal readers could, and, when reading in a moving window experiment, he read better with a small window than with a larger one. ${ }^{18}$ Rayner, Murphy, et al. (1989) argued that he had a selective attention deficit, which made it difficult for him to focus attention on the fixated word, but that he was atypical of dyslexic readers.

In concluding this section, the question arises as to why there is so much inconsistency in the results of experiments on dyslexia and eye movements. Why, for example, did Pavlidis find evidence for erratic eye movements in nonreading tasks while other investigators found no such evidence? There are many reasons why inconsistent results may be obtained with a group of abnormal participants and variability is generally greater with abnormal than normal participants. However, one likely possibility (Morris \& Rayner, 1991; Pollatsek, 1983; Rayner, 1983, 1985 a) is that a subgroup of dyslexic readers have erratic eye movements as found by Pavlidis. Indeed, case studies of dyslexic readers reported by Zangwill and Blakemore (1972) and Pirozzolo and Rayner $(1978,1979)$ have suggested that some dyslexic readers' eye movement characteristics are in close agreement with those reported by Pavlidis. ${ }^{19}$ Likewise, as noted, the dyslexic reader reported by Rayner, Murphy, et al. (1989) was consistent with data reported by Geiger and Lettvin (1987). However, it is not necessarily the case that eye movements per se are the problem. Rather, the evidence suggests that these dyslexic readers have a visual-spatial deficit and that eye movements reflect a more serious underlying spatial orientation problem. For the vast majority of dyslexic individuals, the evidence suggests that they have a language processing deficit and that their eye movements simply reflect their difficulty processing language. Three findings are consistent with this conclusion. First, Hyönä and Olson (1995) found that dyslexic readers show the typical word frequency effect in which low-frequency words are fixated longer than high-frequency words. Second, Pirozzolo and Rayner (1978) found that when dyslexic readers with a language processing deficit were given text appropriate for their reading level, their eye movements were much like those of normal readers at that particular age level (see also Olson, Kliegl, \& Davidson, 1983b); when they were given age appropriate text, their eye movement characteristics showed the usual differences from those of normal readers. Third, Rayner (1986) showed that normal children's eye movements (saccade lengths, fixation durations, and the size of the perceptual span) could be made to look characteristic of dyslexic readers' eye movements when they were given text that was too difficult for them. In sum, the most appropriate conclusion remains that eye movements reflect the difficulties that dyslexic individuals have reading and are not the cause of the reading problem.

\section{Eye Movements in Music Reading and Typing}

\section{Music Reading}

Although some classic studies of eye movements while reading music (usually by pianists) were carried out over 50 years ago ( see Weaver, 1943), only recently has interest in the topic reemerged. Weaver found that (a) the number of notes per fixation was about 1.5 , (b) the average fixation duration varied as a function of the difficulty of the music with more difficult selections leading to longer fixations $(460 \mathrm{~ms})$ than easier selections ( $365 \mathrm{~ms}$ ), (c) 7-23\% of all fixations were regressions, (d) the eye-hand span (how far the eyes are ahead of the hand) was variable (but never exceeded a separation of eight successive notes or chords) and was influenced by the difficulty of the selection, and (e) progression along the musical score was accomplished in two ways: When the music was homophonic and chordal, pianists tended to use a vertical fixation sequence in which they alternated vertical movements from one half of the staff to the other half; however, when the music was contrapuntal, there tended to be a series of horizontal saccades followed by a vertical movement and then more horizontal saccades.

\footnotetext{
${ }^{18}$ Actually, he read better when the window was small and there were $x$ s outside the window. If there were other letters outside the window, he had problems reading.

${ }^{19}$ For example, Pirozzolo and Rayner (1979) reported a case study of a person who could read text better when it was upside-down (and the eye movements going from right to left) than when it was in its normal orientation (see also Larsen \& Parlenvi, 1984).
} 
An issue that has surfaced recently concerns where musicians fixate in the musical score. Goolsby $(1994 a, 1994 b)$ found that skilled music readers did not fixate directly on each note or item of information that was performed. Rather, they often fixated in blank areas of the musical score. He also found that they seldom fixated on note stems or the bars connecting eighth notes, indicating that the information necessary to acquire the rhythmic aspect of the notation is acquired vertically and horizontally within a fixation. Finally, Goolsby (1994b) reported that melodies in which the notes and other visual information were spaced close together resulted in fewer fixations than less condensed notation. Kinsler and Carpenter (1995) found that as the tempo of performance increased, the average time between saccades decreased but the mean amplitude increased. They also found that musicians' eye movements tended to be unrelated to the execution of the elements of performance. Specifically, some of the eye fixations are "holding" periods when the eyes are waiting for the hands to catch up (see also Goolsby, 1989).

Two other topics have received attention recently: (a) individual differences and (b) the perceptual span. With respect to the former, Goolsby (1994a) compared 12 skilled and 12 less skilled music readers as they read music and hummed the melody. The two groups were compared on (a) progressive fixation duration, (b) number of progressive fixations, (c) progressive saccade length, (d) number of regressive fixations, (e) regressive fixation duration, and (f) regressive saccade length. Goolsby found that the two groups reliably differed only in the duration of progressive fixations and that less skilled readers were much more likely to fixate on notes and rests than skilled readers. On the other hand, Polanka (1995) found little difference in looking patterns as a function of expertise. However, in a study in which skilled musicians, less skilled musicians, and nonmusicians were asked to determine whether two scores were identical or not, Waters, Underwood, and Findlay (1997) found that the skilled readers were able to make the judgment with fewer eye movements and shorter fixations than the other two groups; the nonmusicians made more saccades and longer fixations than the less skilled musicians.

Truitt, Clifton, Pollatsek, and Rayner (1997) recently reported a moving window study in which they examined the perceptual span of pianists. They found that when the window included one measure there was little difference from when the entire line of music was available. On the other hand, a halfmeasure window slowed down performance. Truitt et al. also examined individual differences in eye movements when reading music and found results quite consistent with those reported by Goolsby (1994b): The two groups differed only in average fixation duration. Finally, examination ( see also Rayner \& Pollatsek, 1997) of the eye-hand span yielded results that were quite consistent with Weaver's findings: The eye-hand span ranged from the eyes being 2 beats behind the hand to being 12 beats ahead of the hand (however, the eyes were between 1 and 3 beats ahead of the hand $88 \%$ of the time).

\section{Typing}

Classic studies of eye movements during typing were reported over 50 years ago (Butsch, 1932). More recently, a number of studies have been reported by Inhoff and colleagues (Inhoff,
1991; Inhoff, Briihl, Bohemier, \& Wang, 1992; Inhoff, Calabrese, \& Morris, 1986; Inhoff, Chiu, \& Wang, 1990; Inhoff \& Gordon, 1997; Inhoff, Topolski, \& Wang, 1992; Inhoff \& Wang, 1992). The primary finding reported by Butsch (1932) was that typists, irrespective of skill level, attempt to keep the eyes $1 \mathrm{~s}$ ahead of the currently typed letter. However, Inhoff 's studies have found no support for the 1-s hypothesis. Rather, there was considerable variability in the size of the eye-hand $\operatorname{span}(M=$ 2.8 letters), and it was affected by lexical properties of the text. As in music reading, Butsch and Inhoff have both found that there are periods of time when the eyes wait in place for the hand to catch up.

Inhoff and colleagues also examined the size of the perceptual span. Prior research (Salthouse, 1984; Shaffer, 1973) used a moving window paradigm in which the movement of the window coincided with keypresses. However, the eyes do not move one letter space to the right with each keypress. Thus, Inhoff and Wang (1992) and Inhoff, Brihl, et al. (1992) used the eyecontingent moving window paradigm to determine the size of the span in typing. The results indicated that (a) when fewer than seven letter spaces were visible to the right of fixation, saccade size decreased and (b) interkeypress times increased when fewer than three letter spaces were visible. Thus, the perceptual span and the region from which letter information is used in typing is about half that of reading.

\section{Eye Movements and Visual Search}

In comparison to reading, there have not been nearly as many studies dealing with visual search. In fact, most studies of visual search rely on presenting stimuli for brief exposures, and researchers either assume that viewers do not make eye movements ${ }^{20}$ or their eye movements are monitored to make sure they do not move their eyes (Klein \& Farrell, 1989). Other researchers seem to assume that understanding eye movement behavior in visual search may not be particularly important. However, many studies (Binello, Mannen, \& Ruddock, 1995; Findlay, 1995, 1997; Findlay \& Gilchrist, 1997; Gilchrist, Findlay, \& Heywood, in press; Gould, 1973; Miller, 1978; Motter \& Belky, 1998a, 1998b; Ponsoda, Scott, \& Findlay, 1995; Previc, 1996; Scialfa \& Joffe, 1998; Scialfa, Thomas, \& Joffe, 1994; Scinto, Pillalamarri, \& Karsh, 1986; Staller \& Sekuler, 1980; Togami, 1984; Williams, Reingold, Moscovitch, \& Behrmann, 1997; Zelinsky, 1996; Zelinsky, Rao, Hayhoe, \& Ballard, 1997; Zelinsky \& Sheinberg, 1997) have indicated that eye movement data yield important information about visual search. Indeed, Findlay and Gilchrist (1998) argued that the tradition to pay little attention to eye movements in search research is misguided, and they demonstrated that when viewers are free to move their eyes, no additional covert attentional scanning occurs.

Whereas most studies of eye movements during visual search have examined complex search tasks, some (Findlay, 1997; Ottes, Van Gisbergen, \& Eggermont, 1985; Viviani \& Swensson,

\footnotetext{
${ }^{20}$ Recent studies by Jordan, Patching, and Milner (1998) and Patching and Jordan (in press) have demonstrated that when fixation location is not ensured by an eye-tracking system, central fixations occurred on only $23 \%$ of trials.
} 
1982) involved presenting a fairly simple array to participants and examining the characteristics of the initial saccade as a function of the array. Both accuracy and latency of the initial saccade were influenced by the characteristics of the array. Viviani and Swensson (1982) had participants locate a star-shaped target among 15 disk-shaped distractors located between $4.1^{\circ}$ and $12.7^{\circ}$ eccentricity. When the target was at a small eccentricity, participants located it accurately with a single saccade (see also Findlay, 1997); when the target was more peripheral, wrongly directed initial saccades were common (up to $40 \%$ of the time). In free viewing tasks, the tendency to fixate the object closest to fixation is very coercive (Engel, 1977). Zelinsky et al. (1997) found that in a complex search task that participants initially directed their eyes to the center of the global display and then to the centers of recursively smaller groups of objects until the target was acquired.

When eye movements are recorded during extended search, fixations tend to be longer than in reading. However, there is considerable variability in fixation time and saccade length as a function of the particular search task. Specifically, visual search tasks vary widely, and tasks in which eye movements have been monitored consist of at least the following: search (a) through text or textlike material (Rayner \& Fisher, 1987a, 1987b), (b) with pictorial stimuli (Boersma, Zwanga, \& Adams, 1989; Nodine, Carmody, \& Herman, 1979), (c) with complex arrays such as X-rays (Carmody, Nodine, \& Kundel, 1980, 1981), and (d) with randomly arranged arrays of alphanumeric characters or objects (Zelinsky, 1996). Because the nature of the search task influences eye movement behavior, any statement about visual search and eye movements needs to be qualified by the characteristics of the search task.

\section{The Perceptual Span}

Rayner and Fisher (1987a) used the moving window technique as viewers searched through horizontally arranged letter strings for a specified target letter. ${ }^{21}$ They found that the size of the span varied as a function of the difficulty of the distractor letters. When the distractor letters were made up of dissimilar letters (e.g., the target letter was $b$ and the distractor letters were $v, s, z, c, r, w$, and $n$ ), the perceptual span extended from 3 to 4 letters to the left of fixation to about 15 letters to the right. However, when the distractor letters were similar to the target ( $h, k, d$, and $l$ when the target was $b$ ), the span extended only about 12 letters to the right of fixation. Rayner and Fisher (1987b) found that there were two qualitatively different regions within the span: a decision region, where information about the presence or absence of a target is available, and a preview region, where partial letter information is available but where information on the absence of a target is not available. They also found that the decision region varied from 3 to 4 letters when the target was similar to the distractor letters to 5-6 letters when the target was dissimilar to the distractors. Likewise, they found that the preview region varied from 5-6 letters (to the right of the decision region) when the target was similar to the distractors to 7-8 letters when the target was dissimilar to the distractors.

Bertera and Rayner (1998) had viewers search through a randomly arranged array of letters and digits for the presence of a target letter. They used both the moving window technique and the moving mask technique described earlier. The number of items in the array was held constant, but the size of the display was varied $\left(13^{\circ} \times 10^{\circ}, 6^{\circ} \times 6^{\circ}\right.$, and $\left.5^{\circ} \times 3.5^{\circ}\right)$. In the moving window study, search performance reached asymptote when the window was $5^{\circ}$. The moving mask had a deleterious effect on search time and accuracy; the larger the mask, the longer the search time.

\section{Search Difficulty}

The difficulty of the search task influences eye movements. Zelinsky and Sheinberg (1997; see also Williams et al., 1997) found that fixations were longer, saccades were shorter, and more eye movements were made in serial search tasks (such as searching for a circle among Q-like distractors) than in parallel search tasks (a single Q-like target embedded in a field of circles). A number of studies have found that when distractors are similar to targets, fixation time increases, more fixations are made, and saccade size decreases (Noyes, 1980; R. J. Phillips, 1981; Rayner \& Fisher, 1987a, 1987b). Nattkemper and Prinz (1984) also found that fixation duration is affected by the redundancy or predictability of the array. Finally, Moffitt (1980) demonstrated that the difficulty of the array (complexity and density of the array) generally, though not invariably, influenced the average fixation duration.

\section{Comparisons With Reading}

Some studies have compared eye movements when reading with visual search when viewers search either text or letter strings searching for a target letter while moving their eyes from left to right. Although Vitu et al. (1995) argued that there were many eye movement similarities between the two tasks, Rayner and Fischer (1996) demonstrated that there were important differences between them. In particular, they found that when viewers were asked to search for a target word, the word frequency effect disappeared (see also Rayner \& Raney, 1996). Thus, although readers looked longer at low-frequency words than high-frequency words, when the task was changed to search, there was no difference in how long the two types of words were fixated. Rayner and Fischer (1996; see Vitu et al., 1995) also found that fixations were longer in search than in reading (when the stimulus was the same in the two situations). As noted earlier, readers do not obtain information from below the line they are reading. However, in search tasks in which viewers search through rows of letters, Prinz (1984; Prinz, Nattkemper, \& Ullann, 1992) found that information is obtained from below the fixated line. Although there are some similarities between eye movements in search and reading, there are important differences. Thus, it is hazardous to generalize from search to reading given that the cognitive demands differ in the two cases.

\footnotetext{
${ }^{21}$ In Rayner and Fisher's (1987a) experiments, the search array always consisted of strings of letters, with the string size being either 2 , 4,6 , or 8 letters. Spaces were inserted between the strings (such as pklm jhtr nbfd). The strings were searched from left to right.
} 


\section{Eye Movement Control}

Vaughan and colleagues (Graefe \& Vaughan, 1978; Vaughan, 1982, 1983; Vaughan \& Graefe, 1977) tried to determine whether fixation durations in visual search are influenced by the nature of currently fixated information or whether they are preprogrammed (i.e., programmed without regard to the currently fixated information). Vaughan presented target letters immediately on fixation or else delayed their appearance with a mask, as in the Rayner and Pollatsek (1981) experiment. He concluded that whereas some fixations are preprogrammed, others are influenced by the fixated information. Other results (Hooge \& Erkelens, 1996, 1998) have suggested that the completion of foveal analysis is not necessarily the trigger for the subsequent saccade. Indeed, there is some controversy over how much processing of the fixated target occurs before a saccade (see Sanders \& van Duren, 1998; Viviani, 1990); the amount of processing that is completed may vary as a function of the nature of the specific search task under investigation. In general, though, it seems reasonable that in a visual search paradigm, the link between eye fixations and cognitive processes might not be as tight as they are in reading. Nevertheless, cognitive load does increase fixation time (Findlay \& Kapoula, 1992).

Jacobs $(1986,1987 \mathrm{a}, 1987 \mathrm{~b}, 1987 \mathrm{c}, 1991$; Jacobs \& O'Regan, 1987; see also O'Regan et al., 1983) examined the extent to which the visual span influences eye movements. The visual span was defined as the size of the region around fixation in which letters can be recognized with a given accuracy without the use of any contextual information. Jacobs (1986) measured the visual span psychophysically when viewers had to find a target letter in a row of $x \mathrm{~s}$ without moving their eyes; the target letter was presented at different eccentricities from fixation on each trial. The eye movement behavior for the same stimuli was then examined and predictions were made concerning how easy the target was to locate on the basis of the visual span determined for that particular target letter. About $80 \%$ of the variance of mean saccade length could be accounted for by adjustments of saccades to changes in visual span, whereas less than $50 \%$ of fixation duration variance was determined by visual span changes. Jacobs concluded that cognitive factors related to decision processes intervened in the triggering of saccades and that the computation of their spatial parameters play an important role in determining fixation durations in a simple search task. In reading, something like lexical access presumably serves as the trigger to move the eyes from one location to another, but in visual search, a simpler process may well serve as the trigger. Indeed, Rayner (1995) suggested that the trigger to move the eyes in a search task is something like "Is the target present in the decision region?" If it is not, a new saccade is programmed to move the eyes to a location that has not been examined. As with reading, attention would move to the region targeted for the next fixation prior to the actual saccade. Theeuwes, Kramer, Hahn, and Irwin (1998) recently reported evidence consistent with parallel programming of two saccades: one voluntary, goaldirected eye movement to a search target and one reflexive, saccade elicited by the appearance of a new object.

The decision about where to fixate next is also strongly influenced by the characteristics of the specific search task. When searching through text or textlike arrays, the eyes would be programmed to move just beyond the decision region into the preview region (Rayner \& Fisher, 1987b). How far the eyes actually move would be determined by the characteristics of the array (dense, difficult distractors, etc). In situations such as this, the participant would primarily be moving from left to right across the line. Jacobs (1987b) found that when letter strings had spaces between them (as in Rayner \& Fisher, 1987b), participants actually moved their eyes a shorter distance than when there were no spaces. He also found that average fixation duration and overall search rate were longer when there were spaces. When the array consists of randomly arranged alphanumeric characters (Bertera \& Rayner, 1998) or colored bars (Zelinsky, 1996) or circles with or without line segments (Williams et al., 1997; Zelinsky \& Sheinberg, 1997), then more effort is needed because a strategy must be developed for systematically examining the array.

\section{Eye Movements and Scene Perception}

In comparison to visual search, there have been many recent studies examining scene perception. Although there has not been as much research on this subject as there has been on reading, many of the same questions have been addressed within the context of scene perception.

\section{How Important Are Eye Movements in Scene Perception?}

Viewers make many eye movements when looking at a scene. Despite this simple fact, it has often been implied that examining the fine details of eye movements during scene perception is a high-cost, low-yield endeavor. Some experiments using tachistoscopic presentations (see Biederman, Mezzanotte, \& Rabinowitz, 1982) and eye movement recordings (see G. R. Loftus \& Mackworth, 1978) have led to the conclusion that participants get the gist of a scene very early in the process of looking, sometimes even from a single brief exposure. Thus, it has been advocated that the gist of the scene is abstracted on the first couple of fixations, and the remainder of the fixations on the scene are used to fill in details. Given these findings, one may question the value of information gleaned from detailed eye movement analyses as people look at scenes.

Although this seems like a reasonable point, it basically assumes that the conclusions reached in the studies are valid. However, as Rayner and Pollatsek (1992) argued, it remains to be conclusively demonstrated that tachistoscopic studies reveal a perceptual effect rather than the outcome of memory processes or guessing strategies. Likewise, concerns can be generated about the eye movement studies that have supported the idea that the gist is abstracted very quickly from a scene. One finding is that the eyes are quickly drawn to informative regions of a picture (Antes, 1974; Mackworth \& Morandi, 1967). Another prominent finding is that the eyes quickly move to an object that is out of place in a scene (Friedman, 1979; Friedman \& Liebelt, 1981; G. R. Loftus \& Mackworth, 1978). These findings have been taken as evidence that viewers quickly get the gist of the scene and move their gaze to the object that does not fit. However, Rayner and Pollastek (1992) pointed out that the eyes 
may be quickly drawn to informative areas because they are physically distinctive in the scenes, and the definition of what is informative in these studies often corresponds to a definition of what is distinctive. Thus, in studies in which the eyes quickly move to an out-of-place object, it is also possible that such objects are physically distinct from the surroundings. In recent experiments, DeGraef, Christiaens, and d'Ydewalle (1990); DeGraef (1998); and Henderson, Weeks, and Hollingworth (in press ) controlled for distinctiveness, and none of them replicated the finding that semantically inconsistent objects were fixated earlier than consistent objects. It is, however, clear that important or interesting objects in scenes are fixated more and longer than less important objects (Christianson, Loftus, Hoffman, \& Loftus, 1991; Henderson et al., in press; Kristjanson \& Antes, 1989; G. R. Loftus \& Mackworth, 1978; E. F. Loftus, Loftus, \& Messo, 1987).

A controversial claim regarding the importance of eye movements was advocated by Noton and Stark (1971a, 1971b; for more recent work see Choi, Mosley, \& Stark, 1995; Ellis \& Stark, 1986; Stark \& Ellis, 1981; Zangemeister, Sherman, \& Stark, 1995) in their "scanpath" theory in which the process of pattern perception is viewed as a serial process with a fixedorder strategy of extraction of information from the scene during an initial viewing period and during subsequent recognition of the scene. Although the sequence of fixations is largely unpredictable before viewing begins, Noton and Stark argued that memory for a scene consists of information about the sequence actually used to get the information in the first place. According to their theory, the order of fixations on a scene during initial viewing and later recognition should be similar, and they presented data consistent with this idea. However, although it has been demonstrated a number of times that the initial viewing and subsequent recognition patterns of fixations are often similar (Mannen, Ruddock, \& Wooding, 1995, 1996, 1997), other studies (Locher \& Nodine, 1974; Parker, 1978; Walker-Smith, Gale, \& Findlay, 1977) indicated that there is no necessity that they be so for accurate recognition to occur.

G. R. Loftus (1972; Christianson et al., 1991) found that memory for a scene was related to the number of fixations made on the scene: More fixations yielded higher recognition scores. ${ }^{22}$ Loftus's general finding, along with the fact that there is quite a bit of variation in the pattern of eye fixations on a scene as a function of instructions (Buswell, 1935; Yarbus, 1967), suggests that people continue to abstract important information from the scene following the initial few fixations. Antes (1974) reported a pattern of visual exploration whereby participants initially made many long saccades to fixate on informative parts of the scene with short fixations. This behavior gradually evolved into fixating informative areas less frequently and examining less informative details through short saccades and long fixations. On the other hand, G. R. Loftus (1983) found that the initial fixation on a scene was shorter than subsequent fixations, and Nodine, Carmody, and Kundel (1978) found no evidence that mean fixation duration or saccade length varied over the course of viewing. Fixations were longer, however, on areas rated high in informativeness.

Finally, van Diepen, DeGraef, and d'Ydewalle (1995) used the moving mask paradigm to investigate how quickly information in a scene can be obtained. Viewers examined scenes with a pattern mask presented at fixation either $15,45,75$, or 120 ms after the onset of a fixation. On a given trial, the mask was either $1.5^{\circ}$ wide and $1^{\circ}$ high or $2.5^{\circ}$ wide and $1.7^{\circ}$ high. In a control condition, no mask was presented. Total viewing time was longer when a mask was present than when it was not only in the 15-ms delay condition. Thus, van Diepen et al. concluded that the information needed for object analysis was acquired within the first $45-75 \mathrm{~ms}$ of a fixation. However, their fixation duration data led them to conclude that, as in reading, information can be acquired throughout the fixation as needed.

Given the existing data, there is fairly good evidence that information is abstracted throughout the time course of viewing a scene (G. R. Loftus, 1981, 1983). Whereas the gist of the scene is obtained early in viewing, useful information from the scene is obtained after the initial fixations. Thus, important conclusions about the temporal aspects of scene perception can be obtained by analyses of eye movements.

\section{The Perceptual Span}

Rayner and Pollatsek (1992) argued that the data on the perceptual span in scene perception mirrors that for reading with one important difference: Meaningful information can be extracted much further from fixation in scenes than in text. Using a moving mask paradigm, Henderson, McClure, Pierce, and Schrock (1997) confirmed this conclusion and showed that although the presence of a foveal mask influences looking time, it does not have nearly as serious effects for object identification as a foveal mask has for reading. Although these data confirm that objects can be identified farther from fixation than words, two other lines of research suggest that there are limits to how far from fixation information can be extracted even in scene perception. First, Nelson and Loftus (1980) determined how close to fixation an object had to be for it to be recognized as being in the scene. They found that objects located within about $2.6^{\circ}$ from fixation were generally recognized, but recognition depended to some extent on the characteristics of the object. They also suggested that qualitatively different information is acquired from the region $1.5^{\circ}$ around fixation than from any region further from fixation. Similar results were reported by Nodine, Carmody, and Herman (1979). Second, Saida and Ikeda (1979) used the moving window paradigm and varied the window size and the exposure duration of a scene; the probability of correct recognition was determined as a function of these two variables. In addition, two picture sizes were used $\left(14.4^{\circ}\right.$ $\times 18.8^{\circ}$ and $\left.10.2^{\circ} \times 13.3^{\circ}\right)$. As would be expected, and consistent with G. R. Loftus (1972), recognition accuracy increased with viewing duration. More important, Saida and Ikeda found a serious deterioration in recognition when the window was limited to a small area (about $3.3^{\circ} \times 3.3^{\circ}$ ) on each fixation. Performance gradually improved as window size became larger, reaching asymptote when it was about $50 \%$ of the entire picture. Saida and Ikeda noted that the scene is scanned so that there is considerable overlap of information across fixations.

\footnotetext{
${ }^{22}$ Tversky (1974) found just the opposite effect: Fewer fixations (but longer fixations) were associated with better recall and recognition of pictures.
} 
Shioiri and Ikeda (1989) varied window size while the information outside of the window was degraded by adding visual noise. They tried to find the critical window size beyond which a further increase did not improve performance. They found that the greater the degradation, the larger the window had to be to meet the criterion performance. Thus, at high eccentricities, severely degraded information yielded normal performance. Shioiri and lkeda concluded that low-resolution information is processed in the more peripheral parts of the visual field, whereas high-resolution information is processed in foveal vision. More recently, Wampers, van Diepen, and d'Ydewalle (1998) used a moving window paradigm ( see van Diepen, 1997; van Diepen, Wampers, \& d'Ydewalle, 1998) and found that high spatial frequency information is more useful in parafoveal and peripheral vision than low spatial frequency information.

\section{Integration of Information Across Saccades}

How information is combined across saccades and how we perceive a visually constant world in spite of the displacement of the light pattern on the retina with each saccade has been the topic of much debate (Bridgeman, van der Heijden, \& Velichkovsky, 1994; Irwin, 1992b, 1993, 1996; Pollatsek \& Rayner, 1992). As with reading, it has been suggested that information is integrated in a visual buffer that combines the information available on successive fixations (Breitmeyer, 1983; Feldman, 1985; Jonides, Irwin, \& Yantis, 1982). However, research on object perception has found that visual information is not overlapped in a point-by-point manner in a buffer. The typical paradigm used in this research involves eye contingent display changes (see Pollatsek et al., 1984). Viewers' eye movements are monitored, and an object is presented in extrafoveal vision. Viewers are instructed to move their eyes to the location of the object, and during the saccade, the initially presented object is replaced by another object that the participant must name (with the relationship between the two objects being varied). As in reading, viewers obtain information from an extrafoveal object that aids them in naming the target object. This preview effect is about $100 \mathrm{~ms}$ both in the naming paradigm (Henderson, 1992a, 1992b; Henderson, Pollatsek, \& Rayner, 1987; Pollatsek et al., 1984; Pollatsek, Rayner, \& Henderson, 1990) and when target fixation time is measured (Henderson, Pollatsek, \& Rayner, 1989).

A number of interesting results have emerged from this paradigm. First, a size change of $10 \%$ produced no decrement in preview benefit (Pollatsek et al., 1984), and there was preview benefit when the location of the preview and target changed (Pollatsek et al., 1990), indicating that a direct point-to-point mapping was irrelevant for preview benefit. Second, there was some preview benefit when the preview and target shared the same name (e.g., a baseball bat and the animal bat), indicating that phonological codes may be involved in the integration process (Pollatsek et al., 1984). Third, changing the contour information of an object from one fixation to another influenced the amount of preview benefit (Henderson, 1997). Fourth, changing the form of the preview but preserving the meaning also altered the preview benefit. That is, the benefit was significantly smaller when one dog (e.g., a collie) was the preview object and a different dog (a German shepherd) was the target than when the preview and target were identical. Thus, integration is taking place at a level less abstract than the basic level concept. Another finding that indicated something less abstract than the meaning was involved in the integration process was that a visually similar preview was facilitory ( such as a carrot preview for a baseball bat), whereas a semantically similar preview ( such as a baseball preview for a baseball bat) was not (Pollatsek et al., 1984). This latter finding suggests that visual form in some sense is mediating the integration across saccades. In addition, Henderson and Seifert (in press-a, in press-b) and Pollatsek et al. (1984) found that left-right orientation of an object is retained and integrated across a saccade in object identification. ${ }^{23}$

A number of studies (Blackmore, Brelstaff, Nelson, \& Troscianko, 1995; Carlson-Radvansky \& Irwin, 1995; Henderson, 1992a, 1994; Henderson \& Anes, 1994; Henderson et al., 1987; Henderson \& Seifert, in press-a; Irwin, 1991, 1992a; Irwin \& Andrews, 1996; Irwin \& Gordon, 1998; Juttner, 1997; Juttner \& Rohler, 1993; Palmer \& Ames, 1992; Pollatsek et al., 1984, 1990) have converged on the conclusion that transsaccadic integration of real-world objects relies to a high degree on relatively abstract representations. Apparently, the preview image of an object leads to the computation of an abstract structural description of the object (Carlson-Radvansky, in press; Carlson-Radvansky \& Irwin, 1995; Henderson \& Seifert, in press-a). In general, it does not appear that anything resembling precise spatial alignment is needed for combining information across saccades. Virtually the same preview benefit can be obtained if the preview is presented extrafoveally, followed by the target presented foveally after approximately $200 \mathrm{~ms}$ (which is the average saccade latency in these experiments), with the participant maintaining fixation throughout (Pollatsek et al., 1990). Thus, the integration process does not depend on spatial alignment of the two images. This conclusion is also supported by research demonstrating that a scene can be shifted left or right during a saccade, and displacement of the scene is not detected if the eye movement is more than three times larger than the scene displacement (Bridgeman, Hendry, \& Stark, 1975; Bridgeman \& Stark, 1979).

Consistent with the latter finding are some interesting experiments by McConkie and Currie (1996; see also Irwin, McConkie, Carlson-Radvansky, \& Currie, 1994), which have revealed that participants are remarkably unaware of changes occurring in scenes when they move their eyes. Thus, if participants are looking at a scene that consists of a house and a front yard (with a tree in the front yard), they are often unaware of the tree changing location or even completely disappearing from the scene. Of course, the disappearance of the tree is noticed when it is important, the target for a saccade, or attention is drawn to it (see Irwin \& Gordon, 1998), but McConkie and Currie's finding is consistent with the idea that abstract features of the scene are encoded. McConkie and Currie and Irwin et al. proposed the saccade target theory to account for the results.

\footnotetext{
${ }^{23}$ Henderson and Seifert (in press-a) obtained similar results in the naming paradigm and when fixations were monitored during a scanning task. Henderson and Seifert (in press-b) also found that left-right orientation mediates the preview benefit only when position remains constant. They also found greater preview benefit when objects remain constant than when they change location.
} 
According to this theory, prior to a saccade a target object in a scene is chosen. Once this object is chosen, the goal of the perceptual system is to encode critical features about the object. After the saccade, these features are used by the system to search for the target object in the visual array provided during the new fixation. Finding the object provides the bridge that coordinates perception across saccades. Because the search for the object is limited to a narrow region around the fovea, saccadic stimulus shifts are detected if the shift places the target object outside of the limited search region. However, if the target object is close enough to fixation, changes in the scene are not detected (see Deubel, Bridgeman, \& Schneider, 1998; Deubel, Schneider, \& Bridgeman, 1996, for similar arguments).

When the stimuli do not involve meaningful properties, is low-level visual information integrated across saccades? Some experiments purported to demonstrate such low-level visual integration, but the results were artifactual. In particular, Jonides, Irwin, and Yantis (1982) reported some experiments that were initially taken as evidence for a low-level integrative visual buffer. In these experiments, participants were asked to fixate on a target in the center of vision while in parafoveal vision, 12 dots appeared. The 12 dots appeared to be a random array, but in reality they formed part of a $5 \times 5$ matrix. Participants were instructed to move their eyes to the array, and when they did so, the 12 initially presented dots were replaced by 12 different dots. The participants' task was to indicate which dot from the $5 \times 5$ array was missing. Clearly, this task could be done only if participants were holding the first 12 dots in a buffer and integrating that information with the second 12 dots available following the saccade. Jonides et al. found that the location of the missing dot was reported with an extremely high degree of accuracy. In contrast, performance in a control condition (in which participants held fixation while the first 12 dots were presented parafoveally and then the second 12 dots were presented foveally) was at chance level. These results thus seemed like good evidence for a low-level integrative visual buffer. Other studies (Breitmeyer, Kropfl, \& Julesz, 1982; Ritter, 1976; Wolf, Hauske, \& Lupp, 1978, 1980) dealing with low-level visual processing reported findings consistent with the integrative visual buffer theory. However, a number of studies (Bridgeman \& Mayer, 1983; Irwin, Yantis, \& Jonides, 1983; Jonides, Irwin, \& Yantis, 1983; O'Regan \& Lévy-Schoen, 1983; Rayner \& Pollatsek, 1983) demonstrated that the original results were due to screen persistence in the display monitor used to present the stimuli. When appropriate controls were used, the effect was small or nonexistent. Some careful experiments by Irwin (1991, 1992a; Irwin, Zacks, \& Brown, 1990) demonstrated that the results of Breitmeyer et al., Ritter, and Wolf et al. were artifactual because of (a) inadequate control over stimulus presentation, (b) screen persistence on the displays used to present stimuli, and (c) comparison with inappropriate control conditions (see Irwin, 1993).

A phenomenon that would be consistent with an integrative visual buffer is if backward masking occurred across fixations. That is, if a stimulus appeared in a spatial location and a mask appeared in the same spatial location on the subsequent fixation, would the mask affect the visibility of the stimulus? Davidson, Fox, and Dick (1973) reported that there was a cross-saccade effect of retinotopic masking (i.e., that a later mask in the same retinal location inhibited identification of the stimulus) and that the mask in the same spatial location as the target appeared phenomenally to cover it but did not actually degrade the information. McRae, Butler, and Popiel (1987) obtained evidence of retinotopic and spatiotopic masking associated with eye movements. Later work by Irwin, Brown, and Sun (1988) indicated that the retinal masking phenomenon can be replicated, but that there was little evidence that the mask in the same spatial location has any effect either informationally or phenomenally on the visability of the target stimulus ( see also Van der Heijden, Bridgeman, \& Mewhort, 1986).

In summary, the evidence suggests that when meaningful information is presented, integration of information occurs at an abstract level of representation rather than at a visual level (see Irwin, 1992b, 1993; Pollatsek \& Rayner, 1992). Although some very low-level visual information may be integrated across saccades in some circumstances ( see Hayhoe, Bensinger, \& Ballard, 1998; Hayhoe, Lachter, \& Feldman, 1991; Hayhoe, Lachter, \& Moeller, 1992), when meaningful processing occurs (as in scene perception), the information that survives the saccade is at a higher level of representation than pure visual information.

\section{Scene Context Effects on Eye Movements}

Scene context has an effect on eye movements: Fixation time on an object that belongs in a scene is less than fixation time on an object that does not belong (Antes \& Penland, 1981; Friedman, 1979; Henderson et al., in press; G. R. Loftus \& Mackworth, 1978). This result demonstrates that eye movements reflect on-line processing of the scene. However, it is not clear whether the longer fixations on objects in violation of the scene reflect longer times to identify those objects or longer times to integrate them into a global representation of the scene (it could also reflect amusement of the absurdity of the violating object in the given context). Other experiments have therefore attempted to determine whether context operates at the level of object identification. Boyce and Pollatsek (1992) developed a paradigm in which the participant fixates in the middle of a scene, and then after $75 \mathrm{~ms}$ an object wiggles (moves a fraction of a degree of visual angle and then returns to its original position). The participant's task was to name the wiggled object. Participants invariably moved their eyes to the wiggled object, and it was named more rapidly when it was in a normal scene context than when it was in the wrong scene. DeGraef et al. (1990) used an object decision task in which participants examined a scene and pushed a button whenever they detected a nonobject (a geometric entity that they had not seen before) in the scene. DeGraef et al. found that scene context affected fixation time on an object, but only after the scene had been viewed for a while (roughly 10 fixations). Thus, it appears that scene context can have an immediate effect on abject processing (Boyce \& Pollatsek, 1992), but it need not.

\section{Eye Movement Control}

In comparison to what is known about eye movement control in reading, considerably less is known about the mechanisms that control eye movements during scene perception. Consistent with reading studies, Shiori (1993) found that the onset of a 
visual mask (either a uniform mask or a blurred image of the scene) at the onset of a fixation increased fixation duration proportional to the mask delay (see also van Diepen, 1998b), and van Diepen (1998a, 1998b), using an eye-contingent masking technique, found that sufficient information was encoded in the first 50-75 ms of a fixation for object identification to occur. Also, like reading, Henderson (1993) found that viewers tended to fixate near the center of an object. Landing variability was greater in the direction of the eye movement vector than in the direction perpendicular to the movement. He also found that there was more of a tendency to undershoot the center of the object than to overshoot it. Furthermore, landing position influenced fixation time: The duration of the first fixation on an object decreased and the probability of refixating an object increased as the deviation of the initial landing position from the center of the object increased. Mannen et al. $(1995,1996,1997)$ had viewers look at normal scenes or the scenes after they had been either high-pass or low-pass filtered. They found that viewers looked in pretty much the same locations across the three versions of the scene. Because it was virtually impossible to recognize the scenes in the nonnormal situations, low-level visual information must be critical in determining where to fixate next (Henderson, 1992b).

With respect to when to move the eyes, low-level variables, such as luminance and contrast, influence fixation time on a scene (G. R. Loftus, 1985; G. R. Loftus, Kaufman, Nishimoto, \& Ruthruff, 1992). However, more critically, some aspect of object recognition is related to fixation time, ${ }^{24}$ because (a) the eyes move from an object quickly when a semantically related object was just fixated (DeGraef, 1992; DeGraef, DeTroy, \& d'Ydewalle, 1992; Henderson, 1992a; Henderson et al., 1987, 1989), (b) incongruous objects in scenes are fixated longer than congruous objects (Antes \& Penland, 1981; Friedman, 1979; Henderson et al., in press; G. R. Loftus \& Mackworth, 1978), and (c) important objects are fixated longer than less important ones (Christianson et al., 1991; E. F. Loftus et al., 1987).

Ideally, a model of eye movement control during scene perception should specify the mechanisms involved both in when to move the eyes and where to move the eyes. However, because eye movements in scene perception depend on the task (Buswell, 1935; Rayner \& Pollatsek, 1992; Yarbus, 1967), this is not a trivial endeavor. Henderson (1992b; Henderson \& Hollingworth, 1998; Henderson et al., in press) and Rayner and Pollatsek (1992) have proposed that a model like Morrison's model of reading can account for eye movements during scene perception. Thus, something like identification of the fixated object (or objects) serves as a trigger to move to another part of the scene. Exactly where to move the eyes is probably driven by low-level information (Findlay, 1981, 1982; Findlay, Brogan, \& Wenban-Smith, 1993; He \& Kowler, 1989; Henderson et al., in press; Mannen et al., 1997; McGowan, Kowler, Sharma, \& Chubb, 1998), with the center of tobe-fixated objects or other prominent aspects of the scene serving as targets for the next fixation. Certainly, if something moves (as in Boyce \& Pollatsek's, 1992, experiments), the movement drives the eyes to that location. However, even with static displays, it is plausible that lower level information usually dominates eye control. Because viewers concentrate on processing the fixated information, it would make sense to leave the job of deciding where to fixate next to a relatively "dumb" process operating on information in parafoveal and peripheral vision, such as brightness differences or contours, that use little or no central processing capacity.

\section{Eye Movements and Other Information Processing Tasks}

In addition to the topics discussed above, eye movements have also been examined in other information processing domains. It is beyond the scope of the present review to describe all of this research in detail, but examples of a number of tasks are described.

\section{Auditory Language Processing}

A currently popular method to study auditory language processing involves recording eye movements as people listen to a story or follow instructions regarding an array they are looking at. Cooper (1974) introduced this method and found that when viewers listen to spoken language with an array in front of them consisting of elements that occur in the discourse that they quickly move their eyes to those elements that are most closely related to the meaning of the words currently heard. Tanenhaus and colleagues (Allopena, Magnuson, \& Tanenhaus, 1998; Eberhard, Spivey-Knowlton, Sedivy, \& Tanenhaus, 1995; Tanenhaus \& Spivey-Knowlton, 1996; Tanenhaus, Spivey-Knowlton, Eberhard, \& Sedivy, 1995, 1996) and others (Meyer, Sleiderink, \& Levelt, 1998) have used the method to examine a number of different issues. Lansing and McConkie (1994) advocated recording eye movements of deaf speech readers as a means of studying the visual perception of spoken language, and Vatikiotis-Bateson, Eigsti, Yano, and Munhall (1998) examined eye movements during audio-visual speech perception.

\section{Mathematics, Numeral Reading, and Problem Solving}

Eye movements have also been recorded as participants solve math and physics problems (De Corte, Verschaffel, \& Pauwels, 1990; Hegarty \& Just, 1993; Hegarty, Mayer, \& Green, 1992; Suppes, 1990; Suppes, Cohen, Laddaga, Anliker, \& Floyd, 1983; Verschaffel, De Corte, \& Pauwels, 1992), as well as analogies (Bethell-Fox, Lohman, \& Snow, 1984; Carpenter, Just, \& Shell, 1990; Dillon, 1985). Not surprisingly, more complicated aspects of the problems typically lead to more and longer fixations. Studies dealing with numeral reading (Brysbaert, 1995; Chincotta et al., 1997; Gielen, Brysbaert, \& Dhondt, 1991; Pynte, 1974) have revealed that fixations on numbers are influenced by the number of syllables in the numeral and by the frequency and magnitude of the numeral. Research on these topics has yielded some interesting observations although the eye movement data are usually more descriptive than the data in reading, visual search, and scene perception.

\footnotetext{
${ }^{24}$ It is interesteing that Zangemeister et al. (1995) found that eye movement patterns were fairly similar when viewing expressionist art (in which there are typically no well-defined objects) and scenes.
} 


\section{Eye Movements and Dual Tasks}

Pashler, Carrier, and Hoffman (1993) examined eye movements when viewers were engaged in a dual-task situation. For example, a speeded manual choice response to a tone was made in close proximity to an eye movement. Although there was some slowing of the eye movement, the dual-task situation did not yield the dual-task interference effect typically found. Jacobs, Nazir, and Heller (1989) presented pairs of letters in peripheral vision, and participants had to judge whether the two were the same. On the basis of saccade-latency data, a confusability matrix of letters was established. Eye movements have also been recorded as indices of mental workload (May, Kennedy, Williams, Dunlop, \& Brannan, 1990; Moray \& Rotenberg, 1989), concurrent memory load (Donk, 1994; Roberts, Hager, \& Heron, 1994), and in conjunction with verbal protocols (J. P. Hansen, 1991) in problem-solving situations. Finally, eye movements have been examined in tasks in which viewers count dots or small lines (Kowler \& Steinman, 1977, 1979; Van Oeffelen \& Vos, 1984a, 1984b). Ikeda, Saida, and Sugiyama (1977) used the moving window paradigm in a line comparison task. They found that accurate performance deteriorated if the window was smaller than the comparison lines and concluded that our excellent ability for line comparison is only possible when the two lines can be observed at one time.

\section{Face Perception}

When examining faces, people tend to fixate on the eyes, nose, mouth, and ears (Luria \& Strauss, 1978; Mertens, Siegmund, \& Grusser, 1993; M. L. Phillips \& David, 1997; Walker, Findlay, Young, \& Lincoln, 1996; Walker-Smith et al., 1977). Fixations tend to be longer when comparisons have to be made between two faces rather than when a single face is examined (M. L. Phillips \& David, 1997). V. Brown, Huey, and Findlay (1997) presented faces and scrambled face distractors in a circle around fixation and asked people to move their eyes to the face. Initially, viewers made short latency saccades to the distractors as frequently as to the faces. However, with practice, they quickly learned to delay the saccade and directed a much higher proportion of saccades to the face target.

\section{Dynamic Situations}

Eye movement behavior in a number of dynamic situations, such as driving (A. S. Cohen, 1981; A. S. Cohen \& Studach, 1977; Dishart \& Land, 1998; Land, 1992; Land \& Furneaux, 1997; Land \& Horwood, 1995; Land \& Lee, 1994; Liu, 1998), basketball foul shooting (Vickers, 1995, 1996), golf putting (Vickers, 1992), table tennis (Land \& Furneaux, 1997), baseball (Bahill \& LaRitz, 1984; Shank \& Haywood, 1987), gymnastics (Vickers, 1988), walking in uneven terrain (Patla \& Vickers, 1997), mental rotation (Carpenter \& Just, 1978; Just \& Carpenter, 1985), and interacting with computer screens (Deffner, 1995; Goldberg \& Schryver, 1995; Goolkasian \& Bunt, 1980; Jacob, 1991; Stampe \& Reingold, 1995) have been examined. Studies in which eye-head coordination is important have revealed that although the eyes often move prior to the head, head and eye movements are generally highly coordinated in such tasks. Other studies of eye movements of drivers have shown different looking patterns for experienced versus novice drivers (Chapman \& Underwood, 1998; Crundall, Underwood, \& Chapman, 1998; Mourant \& Rockwell, 1972), as well as effects of the difficulty of the driving situation (McDowell \& Rockwell, 1978; Miura, 1990; Rahimi, Briggs, \& Thom, 1990; Shinar, McDowell, \& Rockwell, 1977). Niemann, Lappe, and Hoffman (1996) asked viewers to look at three-dimensional objects on a rotating turntable and to memorize the objects. A strategy of thoroughly inspecting the object with a series of closely spaced fixations from a limited number of viewpoints led to the best recognition rates. Studies in which eye-hand coordination is important, such as playing video games (Shapiro \& Raymond, 1989), have revealed orderly sequences in which people coordinate looking and action (Ballard, Hayhoe, Li, \& Whitehead, 1992; Ballard, Hayhoe, \& Pelz, 1995; Epelboim, Steinman, Kowler, Edwards, Pizlo, Erkelens, \& Collewijn, 1995). Epelboim et al. found that eye movements in such situations were more efficient when participants had to perform an action than when they simply had to look at the sequence. Finally, Verfaillie (1997; Verfaillie, De Troy, \& Van Rensbergen, 1994) examined transsaccadic memory for biological-motion for point-light walkers. He found that when the walker was the only visible object, displacements were quite difficult to detect.

\section{Illusions and Imagery}

Studies dealing with illusions, such as the Necker cube (Ellis \& Stark, 1978; Kawabata, Yamagami, \& Noaki, 1978; Scotto, Oliva, \& Tuccio, 1990) or ambiguous figures (Gale \& Findlay, 1983; García-Peréz, 1989; Ruggieri \& Fernandez, 1994; Stark \& Ellis, 1981), appear to have declined since my earlier review (Rayner, 1978b). Brandt and Stark (1997) recently found that the pattern of eye movements was similar when viewers looked at checkered diagrams and when they formed images of them from memory. However, fixations tended to be $75-100 \mathrm{~ms}$ longer during imagery than actual looking.

\section{Brain Damage}

With the growth of cognitive neuropsychology and cognitive neuroscience, it is not surprising that there have been many recent studies dealing with the eye movement characteristics of patients with some type of brain damage or clinical disability. Thus, for example, a number of studies have examined the eye movements of patients with scotomas and visual neglect (Behrmann, Watt, Black, \& Barton, 1997; DeLuca, Spinelli, \& Zoccolotti, 1996; Karnath, 1994; Karnath \& Huber, 1992; Kentridge, Heywood, \& Weiskrantz, 1997; Ladavas, Zeloni, Zaccara, \& Gangemi, 1997; Rizzo \& Hurtig, 1992; Walker \& Findlay, 1996; Walker et al., 1996; Walker \& Young, 1996; Zihl, $1995)$ as they engage in reading, visual search, and scene perception. Gilchrist, Brown, and Findlay (1997) reported an interesting case of a woman with congenital, extraocular muscular fibrosis who cannot make eye movements. However, she reads by moving her head in saccadiclike movements. Studies of eye movements of individuals with schizophrenia (Katsanis, Kortenkamp, Iacono, \& Grove, 1997; M. L. Phillips \& David, 1997; A. B. Sereno \& Holzman, 1993) have also revealed qualitative 
and quantitative differences between them and normal individuals.

\section{Summary}

As the present review documents, eye movement data have proved to be very valuable in studying reading and other information processing tasks. In general, it is probably not appropriate to make generalizations from one task to another about what eye movements reveal about cognitive processes. However, much has been learned about the characteristics of eye movements in the various tasks investigated. More critically, eye movement data are very informative in revealing moment-tomoment processing activities in the tasks reviewed here. Thus, it will most likely be the case that the use of eye movement data will continue to be used to investigate and inform us concerning the types of processing activities discussed in the present review.

\section{References}

Abel, L. A., Troost, B. T., \& Dell'Osso, L. F. (1983). The effects of age on normal saccade characteristics and their variability. Vision Research, 23, 33-37.

Abrams, R. A., \& Jonides, J. (1988). Programming saccadic eye movements. Journal of Experimental Psychology: Human Perception and Performance, 14, 428-443.

Abrams, R. A., Meyer, D. E., \& Kornblum, S. (1989). Speed and accuracy of saccadic eye movements: Characteristics of impluse variability in the oculomotor system. Journal of Experimental Psychology: Human Perception and Performance, 15, 529-543.

Abrams, S. G., \& Zuber, B. L. (1972). Some temporal characteristics of information processing during reading. Reading Research Quarterly, 12, 41-51.

Adams, B. C., Clifton, C., \& Mitchell, D. C. (1998). Lexical guidance in sentence processing? Psychonomic Bulletin \& Review, 5, 265-270.

Adler-Grinberg, D., \& Stark, L. (1978). Eye movements, scanpaths and dyslexia. American Journal of Optometry and Physiological Optics, 55, 557-570.

Albrecht, J. E., \& Clifton, C. (1998). Accessing singular antecedents in conjoined phrases. Memory \& Cognition, 26, 599-610.

Allopena, P. D., Magnuson, J. S., \& Tanenhaus, M. K. (1998). Tracking the time course of spoken word recognition using eye movements: Evidence for continuous mapping models. Journal of Memory and Language, 38, 419-439.

Altarriba, J., Kroll, J. F., Sholl, A., \& Rayner, K. (1996). The influence of lexical and conceptual constraints on reading mixed language sentences: Evidence from eye fixation and naming times. Memory \& Cognition, 24, 477-492.

Altmann, G. T. M. (1994). Regression-contingent analyses of eye movements during sentence processing: A reply to Rayner and Sereno. Memory \& Cognition, 22, 286-290.

Altmann, G. T. M., Garnham, A., \& Dennis, Y. (1992). Avoiding the garden path: Eye movements in context. Joumal of Memory and Language, 31, 685-712.

Altmann, G. T. M., Garnham, A., \& Henstra, J. A. (1994). Effects of syntax in human sentence parsing: Evidence against a structure-based proposal mechanism. Journal of Experimental Psychology: Learning, Memory, and Cognition, 20, 209-216.

Altmann, G. T. M., van Nice, K., Garnham, A., \& Henstra, J. A. (1998). Late closure in context. Journal of Memory and Language, 38, 459484.

Antes, J. R. (1974). The time course of picture viewing. Journal of Experimental Psychology, 103, 62-70.
Antes, J. R., \& Penland, J. G. (1981). Picture context effects on eye movement patterns. In D. F. Fisher, R. Monty, \& J. Senders (Eds.), Eye movements: Cognition and visual perception (pp. 157-170). Hillsdale, NJ: Erlbaum.

Aslin, R. N., \& Shea, S. L. (1987). The amplitude and angle of saccades to double-step target displacements. Vision Research, 27, 1925-1942.

Bahill, A. T., \& LaRitz, T. (1984). Why can't batters keep their eyes on the ball? American Scientist, 72, 249-253.

Bains, R. A., Crawford, J. D., Cadera, W., \& Vilis, T. (1992). The conjugacy of human saccadic eye movements. Vision Research, 32, 1677-1684.

Ballard, D. H., Hayhoe, M. M., Li, F., \& Whitehead, S. D. (1992). Head-eye coordination during sequential tasks. Philosophical Transactions of the Royal Society of London B, 337, 331-339.

Ballard, D. H., Hayhoe, M. M., \& Pelz, J. B. (1995). Memory representation in natural tasks. Journal of Cognitive Neuroscience, 7, 66-80.

Balota, D. A., Pollatsek, A., \& Rayner, K. (1985). The interaction of contextual constraints and parafoveal visual information in reading. Cognitive Psychology, 17, 364-390.

Balota, D. A., \& Rayner, K. (1983). Parafoveal visual information and semantic contextual constraints. Journal of Experimental Psychology: Human Perception and Performance, 9, 726-738.

Bassou, L., Pugh, A. K., Granie, M., \& Morucci, J. P. (1993). Binocular vision in reading: A study of the eye movements of ten-year-old children. In G. d'Ydewalle \& J. Van Rensbergen (Eds.), Perception and cognition: Advances in eye movement research (pp. 295-308) Amsterdam: North Holland.

Beauvillain, C. (1996). The integration of morphological and wholeword form information during eye fixations on prefixed and suffixed words. Journal of Memory and Language, 35, 801-820.

Beauvillain, C., \& Beauvillain, P. (1995). Calibration of an eye movement system for use in reading. Behavior Research Methods, Instruments, \& Computers, 55, 1-7.

Beauvillain, C., \& Doré, K. (1998). Orthographic codes are used in integrating information from the parafovea by the saccadic computation system. Vision Research, 38, 115-123.

Beauvillain, C., Doré, K., \& Baudouin, V. (1996). The "center of gravity" of words: Evidence for an effect of the word-initial letters. Vision Research, 36, 589-603.

Becker, W., \& Jürgens, R. (1979). Analysis of the saccadic system by means of double step stimuli. Vision Research, 19, 967-983.

Behrmann, M., Watt, S., Black, S. E., \& Barton, J. J. S. (1997). Impaired visual search in patients with unilateral neglect: An oculographic analysis. Neuropsychologia, 35, 1445-1458.

Bertera, J. H., \& Rayner, K. (1998). Eye movements and the span of the effective visual stimulus in visual search. Manuscript submitted for publication.

Bethell-Fox, C. E., Lohman, D. F., \& Snow, R. E. (1984). Adaptive reasoning: Componential and eye movement analysis of geometric analogy performance. Intelligence, $8,205-238$.

Biederman, I., Mezzanotte, R. J., \& Rabinowitz, J. C. (1982). Scene perception: Detecting and judging objects undergoing relational violations. Cognitive Psychology, 14, 143-177.

Binder, K. S., \& Morris, R. K. (1995). Eye movements and lexical ambiguity resolution: Effects of prior encounter and discourse topic. Journal of Experimental Psychology: Leaming, Memory, and Cognition, 2I, 1186-1196.

Binder, K. S., Pollatsek, A., \& Rayner, K. (in press). Extraction of information to the left of the fixated word in reading. Journal of Experimental Psychology: Human Perception and Performance.

Binder, K. S., \& Rayner, K. (1998). Contextual strength does not modulate the subordinate bias effect: Evidence from eye fixations and selfpaced reading. Psychonomic Bulletin \& Review, 5, 271-276.

Binello, A., Mannen, S. K., \& Ruddock, K. H. (1995). The characteris- 
tics of eye movements made during visual search with multi-element stimuli. Spatial Vision, 9, 343-362.

Birch, S., \& Rayner, K. (1997). Linguistic focus affects eye movements during reading. Memory \& Cognition, 25, 653-660.

Biscaldi, M., Fischer, B., \& Aiple, F. (1994). Saccadic eye movements of dyslexic and normal reading children. Perception, 23, 45-64.

Black, J. L., Collins, D. W. K., DeRoach, J. N., \& Zubrick, S. (1984a). A detailed study of sequential saccadic eye movements for normaland poor-reading children. Perceptual and Motor Skills, 59, 423-434.

Black, J. L., Collins, D. W. K., DeRoach, J. N., \& Zubrick, S. (1984b). Dyslexia: Saccadic eye movements. Perceptual and Motor Skills, 58, 903-910.

Black, J. L., Collins, D. W. K., DeRoach, J. N., \& Zubrick, S. (1984c). Smooth pursuit eye movements in normal and dyslexic children. Perceptual and Motor Skills, 59, 91-100.

Blackmore, S. J., Brelstaff, G., Nelson, K., \& Troscianko, T. (1995). Is the richness of our visual world an illusion? Transsaccadic memory for complex scenes. Perception, 24, 1075-1081.

Blanchard, H. E. (1985). A comparison of some processing time measures based on eye movements. Acta Psychologica, 58, 1-15.

Blanchard, H. E. (1987). Pronoun processing during fixations: Effects on the time course of information utilization. Bulletin of the Psychonomic Society, 25, 171-174.

Blanchard, H. E., \& Iran-Nejad, A. (1987). Comprehension processes and eye movement patterns in the reading of surprise-ending stories. Discourse Processes, 10, 127-138.

Blanchard, H. E., McConkie, G. W., Zola, D., \& Wolverton, G. S. (1984). The time course of visual information utilization during fixations in reading. Journal of Experimental Psychology: Human Perception and Performance, 10, 75-89.

Blanchard, H. E., Pollatsek, A., \& Rayner, K. (1989). The acquisition of parafoveal word information in reading. Perception \& Psychophysics, 46, 85-94.

Boer, L. C., \& van der Weijgert, E. C. M. (1988). Eye movements and stages of processing. Acta Psychologica, 67, 3-17.

Boersma, T., Zwaga, H. J., \& Adams, A. S. (1989). Conspicuity in realistic scenes: An eye-movement measure. Applied Ergonomics, 20, 267273.

Bouma, H., \& deVoogd, A. H. (1974). On the control of eye saccades in reading. Vision Research, 14, 273-284.

Bowers, A. R., \& Reid, V. M. (1997). Eye movements and reading with simulated impaired vision. Opthalmic and Physiological Optics, 17, $392-402$.

Boyce, S. J., \& Pollatsek, A. (1992). Identification of objects in scenes: The role of scene background in object naming. Journal of Experimental Psychology: Learning, Memory, and Cognition, 18, 531-543.

Brandt, S. A., \& Stark, L. W. (1997). Spontaneous eye movements during visual imagery reflect the content of the visual scene. Journal of Cognitive Neuroscience, 9, 27-38.

Breitmeyer, B. G. (1983). Sensory masking, persistence, and enhancement in visual exploration and reading. In $\mathrm{K}$. Rayner (Ed.), Eye movements in reading: Perceptual and language processes (pp. 330). New York: Academic Press.

Breitmeyer, B. G., Kropfl, W., \& Julesz, B. (1982). The existence and role of retinotopic and spatiotopic forms of visual persistence. Acta Psychologica, 52, 175-196.

Bridgeman, B., Hendry, D., \& Stark, L. (1975). Failure to detect displacement of the visual world during saccadic eye movements. Vision Research, 15, 719-722.

Bridgeman, B., \& Mayer, M. (1983). Failure to integrate visual information from successive fixations. Bulletin of the Psychonomic Society, $21,285-286$.

Bridgeman, B., \& Stark, L. (1979). Omnidirectional increase in thresh- old for image shifts during saccadic eye movements. Perception \& Psychophysics, 25, 241-243.

Bridgeman, B., van der Heijden, L., \& Velichkovsky, B. (1994). A theory of visual stability across saccadic eye movements. Behavioral and Brain Sciences, 17, 247-292.

Briihl, D., \& Inhoff, A. W. (1995). Integrating information across fixations during reading: The use of orthographic bodies and of exterior letters. Journal of Experimental Psychology: Learning, Memory, and Cognition, 21, 55-67.

Britt, M. A., Perfetti, C. A., Garrod, S., \& Rayner, K. (1992). Parsing in discourse: Context effects and their limits. Journal of Memory and Language, 31, 293-314.

Brooks, B. A., Impelman, D. M. K., \& Lum, J. T. (1981). Backward and forward masking associated with saccadic eye movements. Perception \& Psychophysics, 30, 62-70.

Brown, B., Haegerstrom-Portnoy, G., Adams, A. J., Yingling, C. D. Galin, D., Herron, J., \& Marcus, M. (1983). Predictive eye movements do not discriminate between dyslexic and control children. Neuropsychologia, 21, 121-128.

Brown, B., Haegerstrom-Portnoy, G., Yingling, C. D., Herron, J., Galin, D., \& Marcus, M. (1983). Tracking eye movements are normal in dyslexic children. American Journal of Optometry and Physiological Optics, 60, 376-383.

Brown, V., Huey, D., \& Findlay, J. M. (1997). Face detection in peripheral vision: Do faces pop out? Perception, 26, 1555-1570.

Brutten, G. J., Bakker, K., Janssen, P., \& VanderMeulen, S. (1984). Eye movements of stuttering and nonstuttering children during silent reading. Joumal of Speech and Hearing Research, 27, 562-566.

Brysbaert, M. (1995). Arabic number reading: On the nature of the numerical scale and the origin of phonological recoding. Journal of Experimental Psychology: General, 124, 434-452.

Brysbaert, M., \& Mitchell, D. C. (1996). Modifier attachment in sentence parsing: Evidence from Dutch. Quarterly Journal of Experimental Psychology, 49A, 664-695.

Brysbaert, M., \& Vitu, F. (1998). Word skipping: Implications for theories of eye movement control in reading. In G. Underwood (Ed.), Eye guidance in reading and scene perception (pp. 125-148). Oxford, England: Elsevier.

Bullimore, M. A., \& Bailey, I. L. (1995). Reading and eye movements in age-related maculopathy. Optometry and Vision Science, 72, 125138.

Buswell, G. T. (1922). Fundamental reading habits: A study of their development. Chicago: University of Chicago Press.

Buswell, G. T. (1935). How people look at pictures. Chicago: University of Chicago Press.

Butsch, R. L. C. (1932). Eye movements and the eye-hand span in typewriting. Journal of Educational Psychology, 23, 104-121.

Campbell, F. W., \& Wurtz, R. H. (1979). Saccadic omission: Why we do not see a gray-out during a saccadic eye movement. Vision Research, 18, 1297-1303.

Carlson-Radvansky, L. A. (in press). Memory for relational information across eye movements. Perception \& Psychophysics.

Carlson-Radvansky, L., \& Irwin, D. E. (1995). Memory for structural information across eye movements. Journal of Experimental Psychology: Learning, Memory, and Cognition, 21, 1441-1458.

Carmody, D. P., Nodine, C. F., \& Kundel, H. L. (1980). An analysis of perceptual and cognitive factors in radiographic interpretation. Perception, 9, 339-344.

Carmody, D. P., Nodine, C. F., \& Kundel, H. L. (1981). Finding lung nodules with and without comparative visual scanning. Perception \& Psychophysics, 29, 594-598.

Carpenter, P. A., \& Daneman, M. (1981). Lexical retrieval and error recovery in reading: A model based on eye fixations. Journal of Verbal Learning and Verbal Behavior, 28, 138-160. 
Carpenter, P. A., \& Just, M. A. (1978). Eye fixations during mental rotation. In J. W. Senders, D. F. Fisher, \& R. A. Monty (Eds.), Eye movements and the higher psychological functions (pp. 115-133). Hillsdale, NJ: Erlbaum.

Carpenter, P. A., \& Just, M. A. (1983). What your eyes do while your mind is reading. In $\mathrm{K}$. Rayner (Ed.), Eye movements in reading: Perceptual and language processes (pp. 275-307). New York: Academic Press.

Carpenter, P. A., Just, M. A., \& Shell, P. (1990). What one intelligence test measures: A theoretical account of the processing in the Raven Progressive Matrices Test. Psychological Review, 97, 404-431.

Carrithers, C., \& Bever, T. G. (1984). Eye-movement patterns confirm theories of language comprehension. Cognitive Science, 8, 157-172.

Carroll, P. J., \& Slowiaczek, M. L. (1986). Constraints on semantic priming in reading: A fixation time analysis. Memory \& Cognition, $14,509-522$

Carroll, P. J., \& Slowiaczek, M. L. (1987). Models and modules: Multiple pathways to the language processor. In J. L. Garfield (Ed.), Modularity in knowledge representation and natural-language understanding (pp. 221-248). Cambridge, MA: MIT Press.

Carroll, P. J., Young, J. R., \& Guertin, M. S. (1992). Visual analysis of cartoons: A view from the far side. In K. Rayner (Ed.), Eye movements and visual cognition: Scene perception and reading (pp. 444-461). New York: Springer-Verlag.

Cavegn, D., \& d'Ydewalle, G. (1996). Presaccadic attention allocation of express saccades. Psychological Research, 59, 157-175.

Chapman, P. R., \& Underwood, G. (1998). Visual search of dynamic scenes: Event types and the role of experience in viewing driving situations. In G. Underwood (Ed.), Eye guidance in reading and scene perception (pp. 369-394). Oxford, England: Elsevier.

Chekaluk, E., \& Llewellyn, K. R. (1990). Visual stimulus input, saccadic suppression, and detection of information from the postsaccadic scene. Perception \& Psychophysics, 48, 135-142.

Chincotta, D., Hyönä, J., \& Underwood, G. (1997). Eye fixations, speech rate and bilingual digit-span: Numeral reading indexes fluency not word length. Acta Psychologica, 97, 253-275.

Choi, Y. S., Mosley, A. D., \& Stark, L. (1995). String editing analysis of human visual search. Optometry and Vision Science, 72, 439-451.

Christianson, S. A., Loftus, E. F., Hoffman, H., \& Loftus, G. R. (1991). Eye fixations and memory for emotional events. Journal of Experimental Psychology: Learning, Memory, and Cognition, 17, 693-701.

Ciuffreda, K. J. (1979). Jerk nystagmus: Some new findings. American Journal of Optometry and Physiological Optics, 53, 389-395.

Ciuffreda, K. J. (1994). Reading eye movements in patients with oculomotor disturbances. In J. Ygge \& G. Lennerstrand (Eds.), Eye movements in reading (pp. 163-188). Oxford: Pergamon Press.

Ciuffreda, K. J., Kenyon, R. W., \& Stark, L. (1983). Saccadic intrusions contributing to reading disability: A case report. American Journal of Optometry and Physiological Optics, 60, 242-249.

Ciuffreda, K. J., \& Tannen, B. (1995). Eye movement basics for the clinician. St. Louis, MO: C. V. Mosby.

Clifton, C. (1992). Tracing the course of sentence comprehension: How lexical information is used. In $\mathrm{K}$. Rayner (Ed.), Eye movements and visual cognition: Scene perception and reading (pp. 397-414). New York: Springer-Verlag.

Clifton, C. (1993). Thematic roles in sentence parsing. Canadian Journal of Experimental Psychology, 47, 222-246.

Clifton, C., Speer, S., \& Abney, S. P. (1991). Parsing arguments: Phrase structure and argument structure as determinants of initial parsing decision. Journal of Memory and Language, 30, 251-271.

Coëffé, C. (1985). La visée du regard sur un mot isolé [Where does the eye land in isolated words?] Annee Psychologique, 85, 169-184.

Coëffe, C., \& O'Regan, J. K. (1987). Reducing the influence of nontarget stimuli on saccade accuracy: Predictability and latency effects. Vision Research, 27, 227-240.
Cohen, A. S. (1981). Car drivers' pattern of eye fixations on the road and in the laboratory. Perceptual and Motor Skills, 52, 515-522.

Cohen, A. S., \& Studach, H. (1977). Eye movements while driving cars around curves. Perceptual and Motor Skills, 44, 683-689.

Cohen, M. E., \& Ross, L. E. (1977). Saccade latency in children and adults: Effects of warning signals and target eccentricity. Journal of Experimental Child Psychology, 23, 539-549.

Collewijn, H., Erkelens, C. J., \& Steinman, R. M. (1988a). Binocular co-ordination of human horizontal saccadic eye movements. Journal of Physiology, 404, 157-182.

Collewijn, H., Erkelens, C. J., \& Steinman, R. M. (1988b). Binocular co-ordination of human vertical saccadic eye movements. Journal of Physiology, 404, 183-197.

Cooper, R. M. (1974). The control of eye fixation by the meaning of spoken language: A new methodology for the real-time investigation of speech perception, memory, and language processing. Cognitive Psychology; 6, 84-107.

Crawford, T. J. (1990). Multi-stepping saccade sequences in humans. Acto Psychologica, 65, 371-394.

Crawford, T. J. (1996). Transient motion of visual texture delays saccadic eye movement. Acta Psychologica, 92, 251-262.

Crundall, D. W., Underwood, G., \& Chapman, P. R. ( 1998). How much do novice drivers see? The effects of demand on visual search strategies in novice and experienced drivers. In G. Underwood (Ed.), Eye guidance in reading and scene perception (pp. 359-418). Oxford, England: Elsevier.

Daneman, M., \& Reingold, E. (1993). What eye fixations tell us about phonological recoding during reading. Canadian Journal of Experimental Psychology, 47, 153-178.

Daneman, M., Reingold, E., \& Davidson, M. (1995). Time course of phonological activation during reading: Evidence from eye fixations. Journal of Experimental Psychology: Learning, Memory, and Cognition, 21, 884-898.

Davidson, M. L., Fox, M. J., \& Dick, A. O. (1973). Effect of eye movements on backward masking and perceived location. Perception \& Psychophysics, 14, 110-116.

De Corte, E., Verschaffel, L., \& Pauwels, A. (1990). Influence of the semantic structure of word problems on second graders' eye movements. Journal of Educational Psychology, 82, 359-365.

Dee-Lucas, D., Just, M. A., Carpenter, P. A., \& Daneman, M. (1982). What eye fixations tell us about the time course of text integration. In R. Groner \& P. Fraisse (Eds.), Cognition and eye movements (pp. 155-168). Amsterdam: North Holland.

Deffner, G. (1995). Eye movement recordings to study determinants of image quality in new display technology. In J. M. Findlay, R. Walker, \& R. W. Kentridge (Eds.), Eye movement research: Mechanisms, processes, and applications (pp. 479-490). Amsterdam: North Holland.

DeGraef, P. (1992). Scene-context effects and models of real-world perception. In $\mathrm{K}$. Rayner (Ed.), Eye movements and visual cognition: Scene perception and reading (pp. 243-259). New York: SpringerVerlag.

DeGraef, P. (1998). Prefixational object perception in scenes: Objects popping out of schemas. In G. Underwood (Ed.), Eye guidance in reading and scene perception (pp. 313-336). Oxford, England: Elsevier.

DeGraef, P., Christiaens, D., \& d'Ydewalle, G. (1990). Perceptual effects of scene context on object identification. Psychological Research, 52, 317-329.

DeGraef, P., DeTroy, A., \& d'Ydewalle, G. (1992). Local and global contextual constraints on the identification of objects in scenes. Canadian Journal of Experimental Psychology, 46, 489-508.

DeLuca, M., Spinelli, D., \& Zoccolotti, P. (1996). Eye movement patterns in reading as a function of visual field defects and contrast sensitivity. Cortex, 32, 491-502. 
DenBuurman, R., Boersma, T., \& Gerrissen, J. F. (1981). Eye movements and the perceptual span in reading. Reading Research Quarterly, 16, 227-235.

Deubel, H. (1995). Separate addaptive mechanisms for the control of reactive and volitional saccadic eye movements. Vision Research, 35, 3529-3540.

Deubel, H., \& Bridgeman, B. (1995a). Fourth Purkinje image signals reveal eye-lens deviations and retinal image distortions during saccades. Vision Research, 35, 529-538.

Deubel, H., \& Bridgeman, B. (1995b). Perceptual consequences of ocular lens overshoot. during saccadic eye movements. Vision Research, 35, 2897-2902.

Deubel, H., Bridgeman, B., \& Schneider, W. X. (1998). Immediate postsaccadic information mediates space constancy. Vision Research, 38, 3147-3159.

Deubel, H., \& Schneider, W. X. (1996). Saccade target selection and object recognition: Evidence for a common attentional mechanism. Vision Research, 36, 1827-1837.

Deubel, H., Schneider, W. X., \& Bridgeman, B. (1996). Postsaccadic target blanking prevents saccadic suppression of image displacement. Vision Research, 36, 985-996.

Deubel, H., Wolf, W., \& Hauske, G. (1984). The evaluation of the oculomotor error signal. In A. G. Gale \& F. Johnson (Eds.), Theoretical and applied aspects of eye movement research (pp. 55-62). Amsterdam: North Holland.

Deutsch, A. (1998). Subject-predicate agreement in Hebrew: Interrelations with semantic processes. Language and Cognitive Processes, $13,575-597$.

Dillon, R. F. (1985). Eye movement analysis of information processing under different testing conditions. Contemporary Educational Psychology, 10, 387-395.

Di Lollo, V., Bischof, W. F., Walther-Muller, P. U., Groner, M. T., \& Groner, R. (1994). Phosphor persistence in oscilloscopic displays: Its luminance and visibility. Vision Research, 34, 1619-1620.

Dishart, D. C., \& Land, M. F. (1998). The development of eye movement strategies of learner drivers. In G. Underwood (Ed.) Eye guidance in reading and scene penception (pp. 419-430). Oxford, England: Elsevier.

Donk, M. (1994). Human monitoring behavior in multiple-instrument setting: Independent sampling, sequential sampling, or arrangement dependent. Acta Psychologica, 86, 31-55.

Dopkins, S., Morris, R. K., \& Rayner, K. (1992). Lexical ambiguity and eye fixations in reading: A test of competing models of lexical ambiguity resolution. Joumal of Memory and Language, 31, 461477.

Doré, K., \& Beauvillain, C. (1997). Latency dependence of word-initial letter integration by the saccadic system. Perception \& Psychophysics, 59, 523-533.

Duffy, S. A. (1992). Eye movements and complex comprehension processes. In K. Rayner (Ed.), Eye movements and visual cognition: Scene perception and reading (pp. 462-471). New York: SpringerVerlag.

Duffy, S. A., Morris, R. K., \& Rayner, K. (1988). Lexical ambiguity and fixation times in reading. Journal of Memory and Language, 27, $429-446$.

Duffy, S. A., \& Rayner, K. (1990). Eye movements and anaphor resolution: Effects of antecedent typicality and distance. Language and Speech, 33, 103-119.

Dunn-Rankin, P. (1978). The visual characteristics of words. Scientific American, 238, 122-130.

d'Ydewalle, G., \& Gielen, I. (1992). Attention allocation with overlapping sound, image, and text. In K. Rayner (Ed.), Eye movements and visual cognition: Scene perception and reading (pp. 415-427). New York: Springer-Verlag.

Eberhard, K. M., Spivey-Knowlton, M. J., Sedivy, J. C., \& Tanenhaus,
M. K. (1995). Eye movements as a window into real-time spoken language comprehension in natural contexts. Journal of Psycholinguistic Research, 24, 409-436.

Eden, G. F., Stein, J. F., Wood, H. M., \& Wood, F. B. (1994). Differences in eye movements and reading problems in dyslexic and normal children. Vision Research, 34, 1345-1358.

Ehrlich, K. (1983). Eye movements in pronoun assignment: A study of sentence integration. In $\mathrm{K}$. Rayner (Ed.), Eye movements in reading: Perceptual and language processes (pp. 253-268). New York: Academic Press.

Ehrlich, K., \& Rayner, K. (1983). Pronoun assignment and semantic integration during reading: Eye movements and immediacy of processing. Journal of Verbal Learning and Verbal Behavior, 22, 7587.

Ehrlich, S. F., \& Rayner, K. (1981). Contextual effects on word perception and eye movements during reading. Journal of Verbal Learning and Verbal Behavior, 20, 641-655.

Ellis, S. R., \& Stark, L. (1978). Eye movements during the viewing of Necker cubes. Perception. 7, 575-581.

Ellis, S. R., \& Stark, L. (1986). Statistical dependency in visual scanning. Human Factors, 28, 421-438.

Elterman, R. D., Abel, L. A., Daroff, R. B., Dell'Osso, L. F., \& Bornstein, J. L. (1980). Eye movement patterns in dyslexic children. Journal of Learning Disabilities, 13, 312-317.

Engel, F. L. (1977). Visual conspicuity, visual search and fixation tendencies of the eye. Vision Research, 17, 95-108.

Epelboim, J., Booth, J. R., Ashkenazy, R., Taleghani, A., \& Steinman, R. M. (1997). Fillers and spaces in text: The importance of word recognition during reading. Vision Research, 37, 2899-2914.

Epelboim, J., Booth, J. R., \& Steinman, R. M. (1994). Reading unspaced text: Implications for theories of reading eye movements. $V_{i}$ sion Research, 34, 1735-1766.

Epelboim, J., Booth, J. R., \& Steinman, R. M. (1996). Much ado about nothing: The place of space in text. Vision Research, 36, 465-470.

Epelboim, J., Steinman, R. M., Kowler, E., Edwards, M., Pizlo, Z., Erkelens, C. J., \& Collewijn, H. (1995). The function of visual search and memory in sequential looking tasks. Vision Research, 35, 3401-3422.

Eskenazi, B., \& Diamond, S. P. (1983). Visual exploration of non-verbal material by dyslexic children. Cortex, 19, 353-370.

Everatt, J., Bradshaw, M. F., \& Hibbard, P. B. (1998). Individual differences in reading and eye movement control. In G. Underwood (Ed.), Eye guidance in reading and scene perception (pp. 223-242). Oxford, England: Elsevier.

Everatt, J., \& Underwood, G. (1992). Parafoveal guidance and priming effects during reading: A special case of the mind being ahead of the eyes. Consciousness and Cognition, 1, 186-197.

Everatt, J., \& Underwood, G. (1994). Individual differences in reading subprocesses: Relationships between reading ability, lexical access, and eye movement control. Language and Speech, 37, 283-297.

Farmer, M. E., \& Klein, R. M. (1995). The evidence for a temporal processing deficit linked to dyslexia: A review. Psychonomic Bulletin \& Review, 2, 460-493.

Feldman, J. A. (1985). Four frames suffice: A provisional model of vision and space. Behavioral and Brain Sciences, 8, 265-289.

Ferreira, F, \& Clifton, C. (1986). The independence of syntactic processing. Journal of Memory and Language, 25, 348-368.

Ferreira, F., \& Henderson, J. M. (1990). Use of verb information in syntactic parsing: Evidence from eye movements and word-by-word self-paced reading. Journal of Experimental Psychology: Learning, Memory, and Cognition, 16, 555-568.

Ferreira, F., \& Henderson, J. M. (1993). Reading processes during syntactic analysis and reanalysis. Canadian Journal of Experimental Psychology, 47, 247-276.

Ferreira, F., \& McClure, K. K. (1997). Parsing of garden-path sentences 
with reciprocal verbs. Language and Cognitive Pmocesses, 12, 273306.

Fields, H., Wright, S., \& Newman, S. (1993). Saccadic eye movement while reading and tracking in dyslexics, reading-matched, and IQmatched children. In G. d'Ydewalle \& J. Van Rensbergen (Eds.), Perception and cognition: Advances in eye movement research (pp. 309-319). Amsterdam: North Holland.

Findlay, J. M. (1981). Local and global influences on saccadic eye movements. In D. F. Fisher, R. A. Monty, \& J. W. Senders (Eds.) Eye movements: Cognition and visual perception (pp. 171-179). Hillsdale, NJ: Erlbaum.

Findlay, J. M. (1982). Global processing for saccadic eye movements. Vision Research, 22, 1033-1045.

Findlay, J. M. (1992). Programming of stimulus-elicited saccadic eye movements. In K. Rayner (Ed.), Eye movements and visual cognition: Scene perception and reading (pp. 8-30). New York: Springer-Verlag.

Findlay, J. M. (1995). Visual search: Eye movements and peripheral vision. Optometry and Vision Science, 72, 461-466.

Findlay, J. M. (1997). Saccade target selection during visual search. Vision Research, 37, 617-631.

Findlay, J. M., Brogan, D., \& Wenban-Smith, M. G. (1993). The spatial signal for saccadic eye movements emphasizes visual boundaries. Perception \& Psychophysics, 53, 633-641.

Findlay, J. M., \& Gilchrist, I. D. (1997). Spatial scale and saccadic programming. Perception, 26, 1159-1167.

Findlay, J. M., \& Gilchrist, I. D. (1998). Eye guidance and visual search. In $\mathrm{G}$. Underwood (Ed.), Eye guidance in reading and scene perception (pp. 295-312). Oxford, England: Elsevier.

Findlay, J. M., \& Kapoula, Z. (1992). Scrutinization, spatial attention, and the spatial programming of saccadic eye movements. Quarterly Journal of Experimental Psychology, 45A, 633-648.

Findlay, J. M., \& Walker, R. (in press). A model of saccade generation based on parallel processing and competitive inhibition. Behavioral and Brain Sciences.

Fischer, B. (1992). Saccadic reaction time: Implications for reading, dyslexia, and visual cognition. In $\mathrm{K}$. Rayner (Ed.), Eye movements and visual cognition: Scene perception and reading (pp. 31-45). New York: Springer-Verlag.

Fischer, B., Biscaldi, M., \& Otto, P. (1993). Saccadic eye movements of dyslexic adult subjects. Neuropsychologia, 31, 887-906.

Fischer, B., \& Boch, R. (1983). Saccadic eye movements after extremely short reaction times in the monkey. Brain Research, 260, 21-26.

Fischer, B., \& Rampsberger, E. (1984). Human express saccades: Extremely short reaction times of goal directed eye movements. Experimental Brain Research, 57, 191-195.

Fischer, B., \& Weber, H. (1990). Saccadic reaction times of dyslexic and age-matched normal children. Perception, 19, 805-818.

Fischer, B., \& Weber, H. (1993). Express saccades and visual attention. Behavioral and Brain Sciences, 16, 553-567.

Fischer, M. H. (in press). An investigation of attention allocation during sequential eye movement tasks. Quarterly Journal of Experimental Psychology.

Fischer, M. H., \& Rayner, K. (1993). On the functional significance of express saccades. Behavioral and Brain Sciences, 16, 577.

Fisher, D. F., \& Shebilske, W. L. (1985). There is more that meets the eye than the eyemind assumption. In R. Groner, G. W. McConkie, \& C. Menz (Eds.), Eye movements and human information processing (pp. 149-158). Amsterdam: North Holland.

Fletcher, J. M. (1990). Component skill comparisons across reading level and processing demand. Reading Psychology, 11, 193-239.

Fletcher, J. M. (1991). Qualitative descriptions of error recovery patterns across reading level and sentence topic: An eye-movement analysis. Journal of Learning Disabilities, 24, 568-575.

Fletcher, J. M. (1993). Eye-movement rhythmicity and reading comprehension. Journal of Learning Disabilities, 26, 683-688.
Folk, J. R. (1998). Phonological codes are used to access the lexicon during silent reading. Manuscript submitted for publication.

Folk, J. R., \& Morris, R. K. (1995). Multiple lexical codes in reading: Evidence from eye movements, naming time, and oral reading. Journal of Experimental Psychology: Learning, Memory, and Cognition. $21,1412-1429$

Frazier, L., \& Clifton, C. (1998). Comprehension of sluiced sentences. Language and Cognitive Processes, 13, 499-520.

Frazier, L., \& Rayner, K. (1982). Making and correcting errors during sentence comprehension: Eye movements in the analysis of structurally ambiguous sentences. Cognitive Psychology, 14, 178-210.

Frazier, L., \& Rayner, K. (1987). Resolution of syntactic category ambiguities: Eye movements in parsing lexically ambiguous sentences. Journal of Memory and Language, 26, 505-526.

Frazier, L., \& Rayner, K. (1988). Parameterizing the language processing system: Left- vs. right-branching within and across languages. In J. A. Hawkins (Ed.), Explaining language universals (pp. 247-279). Oxford, England: Blackwell.

Frazier, L., \& Rayner, K. (1990). Taking on semantic commitments: Processing multiple meanings vs. multiple senses. Joumal of Memory and Language, 29, 181-200.

Frenck-Mestre, C., \& Pynte, J. (1997). Syntactic ambiguity resolution while reading in second and native languages. Quarterly Journal of Experimental Psychology, 50A, 119-148.

Friedman, A. (1979). Framing pictures: The role of knowledge in automatized encoding and memory for gist. Journal of Experimental Psychology: General, 108, 316-355.

Friedman, A., \& Liebelt, L. S. (1981). On the time course of viewing pictures with a view towards remembering. In D. F. Fisher, R. A. Monty, \& J. W. Senders (Eds.), Eye movements: Cognition and visual perception (pp. 137-156). Hillsdale, NJ: Erlbaum.

Frisson, S., \& Pickering, M. (1998). The processing of metonymy: Evidence from eye movements. Manuscript submitted for publication.

Gale, A. G., \& Findlay, J. M. (1983). Eye movement patterns in viewing ambiguous figures. In R. Groner, C. Menz, D. F. Fisher, \& R. A. Monty (Eds.), Eye movements and psychological functions: Intermational views (pp. 145-168). Hillsdale, NJ: Erlbaum.

García-Peréz, M. A. (1989). Visual homogeneity and eye movements in multistable perception. Perception \& Psychophysics, 46, 397-400.

Garnsey, S. M., Pearlmutter, N. J., Myers, E., \& Lotocky, M. A. (1997). The contributions of verb bias and plausibility to the comprehension of temporarily ambiguous sentences. Joumal of Memory and Language, 37, 58-93.

Garrod, S., Freudenthal, S., \& Boyle, E. (1994). The role of different types of anaphor in the on-line resolution of sentences in a discourse. Journal of Memory and Language, 33, 39-68.

Garrod, S., O’Brien, E. J., Morris, R. K., \& Rayner, K. (1990). Elaborative inferencing as an active or passive process. Journal of Experimental Psychology: Learning, Memory, and Cognition, 16, 250-257.

Geiger, G., \& Lettvin, J. Y. (1987). Peripheral vision in persons with dyslexia. New England Journal of Medicine, 316, 1238-1243.

Geiger, G., Lettvin, J. Y., \& Fahle, M. (1994). Dyslexic children learn a new visual strategy for reading: A controlled experiment. Vision Reseanch, 34, 1223-1233.

Gielen, I., Brysbaert, M., \& Dhondt, A. (1991). The syllable-length effect in number processing is task-dependent. Perception \& Psychophysics, 50, 449-458.

Gilchrist, I. D., Brown, V., \& Findlay, J. M. (1997). Saccades without eye movements. Nature, 390, 130-131.

Gilchrist, I. D., Findlay, J. M., \& Heywood, C. A. (in press). Surface and edge information for spatial integration: A saccade-selection task. Visual Cognition.

Goldberg, J. H., \& Schryver, J. C. (1995). Eye-gaze determination of user intent at the computer interface. In J. M. Findlay, R. Walker, \& 
R. W. Kentridge (Eds.), Eye movement research: Mechanisms, processes and applications (pp. 491-502). Amsterdam: North Holland.

Goolkasian, P., \& Bunt, A. A. (1980). Eye movements and a dynamic stimulus situation. American Journal of Psychology, 91, 251-265.

Goolkasian, P., \& King, J. (1990). Letter identification and lateral masking in dyslexic and average readers. American Journal of Psychology, $103,519-538$.

Goolsby, T. W. (1989). Computer applications to eye movement research in music reading. Psychomusicology, 8, 111-126.

Goolsby, T. W. (1994a). Eye movement in music reading: Effects of reading ability, notational complexity, and encounters. Music Perception, 12, 77-96.

Goolsby, T. W. (1994b). Profiles of processing: Eye movements during sightreading. Music Perception, 12, 97-123.

Gould, J. D. (1973). Eye movements during visual search and memory search. Journal of Experimental Psychology, 98, 184-195.

Grabe, M., Antes, J., Kahn, H., \& Kristjanson, A. (1991). Adult and adolescent readers' comprehension monitoring performance: An investigation of monitoring accuracy and related eye movements. Contemporary Educational Psychology, 16, 45-60.

Grabe, M., Antes, J., Thorson, I., \& Kahn, H. (1987). Eye fixation patterns during informed and uninformed comprehension monitoring. Journal of Reading Behavior, 19, 123-140.

Graefe, T. M., \& Vaughan, J. (1978). Saccadic and manual reaction times to stimuli initiated by eye or finger movements. Bulletin of the Psychonomic Society, 11, 97-99.

Grainger, J., O'Regan, J. K., Jacobs, A. M., \& Sequi, J. (1992). Neighborhood frequency affects and letter visibility in visual word recognition. Perception \& Psychophysics, 51, 49-56.

Groner, R., Groner, M. T., Muller, P. U., Bischof, W. F., \& Di Lollo, $V .(1993)$. On the confounding effects of phosphor persistence in oscilloscopic displays. Vision Research, 33, 913-917.

Hainline, L., Turkel, J., Abramov, I., Lemerise, E., \& Harris, C. M. (1984). Characteristics of saccades in human infants. Vision Research, 24, 1771-1780.

Hallett, P. E. (1978). Primary and secondary saccades to goals defined by instructions. Vision Research, 18, 1279-1296.

Hansen, J. P. (1991). The use of eye mark recordings to supplement verbal retrospective in software testing. Acta Psychologica, 76, 3149.

Hansen, W., \& Sanders, A. F. (1988). On the output of encoding during stimulus fixation. Acta Psychologica, 69, 95-107.

Harris, C. M., Abramov, I., \& Hainline, L. (1984). Instrument considerations in measuring fast eye movements. Behavior Research Methods, Instruments, \& Computers, 16, 341-350.

Harris, C. M., Hainline, L., Abramov, I., Lemerise, E., \& Camenzuli, C. (1988). The distribution of fixation durations in infants and naive adults. Vision Reseanch, 28, 419-432.

Hayhoe, M. M., Bensinger, D. G., \& Ballard, D. H. (1998). Task constraints in visual working memory. Vision Research, 38, 125-137.

Hayhoe, M. M., Lachter, J., \& Feldman, J. (1991). Integration of form across saccadic eye movements. Perception, 20, 393-402.

Hayhoe, M. M., Lachter, J., \& Moeller, P. (1992). Spatial memory and integration across saccadic eye movements. In K. Rayner (Ed.), Eye movements and visual cognition: Scene perception and reading (pp. 130-145). New York: Springer-Verlag.

He, P., \& Kowler, E. (1989). The role of location probability in the programming of saccades: Implications for "center-of-gravity" tendencies. Vision Research, 29, 1165-1181.

He, P., \& Kowler, E. (1992). The role of saccades in the perception of texture patterns. Vision Research, 32, 2151-2163.

Hegarty, M. (1992a). The mechanics of comprehension and comprehension of mechanics. In K. Rayner (Ed.), Eye movements and visual cognition: Scene perception and reading (pp. 428-443). New York: Springer.
Hegarty, M. (1992b). Mental animation: Inferring motion from static displays of mechanical systems. Journal of Experimental Psychology: Learning, Memory and Cognition, 18, 1084-1102.

Hegarty, M., \& Just, M. A. (1993). Constructing mental models of machines from text and diagrams. Journal of Memory and Language, $32,717-742$.

Hegarty, M., Mayer, R. E., \& Green, C. E. (1992). Comprehension of arithmetic word problems: Evidence from students' eye fixations. Journal of Educational Psychology, 84, 76-84.

Heller, D. (1982). Eye movements in reading. In R. Groner \& P. Fraisse (Eds.), Cognition and eye movements (pp. 139-154). Amsterdam: North Holland.

Heller, D. (1983). Problems of on-line processing of EOG-data in reading. In R. Groner, C. Menz, D. F. Fisher, \& R. A. Monty (Eds.), Eye movements and psychological functions: International views (pp. 4352). Hillsdale, NJ: Eribaum.

Heller, D., \& Radach, R. (1995). Binocular coordination in complex visual tasks. Perception, 24, 72 (Supplement).

Henderson, J. M. (1992a). Identifying objects across saccades: Effects of extrafoveal preview and flanker object context. Journal of Experimental Psychology: Learning, Memory, and Cognition, 18, 521-530.

Henderson, J. M. (1992b). Visual attention and eye movement control during reading and picture viewing. In $\mathrm{K}$. Rayner (Ed.), Eye movements and visual cognition: Scene perception and reading (pp. 260 283). New York: Springer-Verlag.

Henderson, J. M. (1993). Eye movement control during visual object processing: Effects of initial fixation position and semantic constraint. Canadian Journal of Experimental Psychology, 47, 79-98.

Henderson, J. M. (1994). Two representational systems in dynamic visual identification. Journal of Experimental Psychology: General, $123,410-426$.

Henderson, J. M. (1997). Transsaccadic memory and integration during real-world object perception. Psychological Science, 8, 51-55.

Henderson, J. M., \& Anes, M. D. (1994). Roles of object-file review and type priming in visual identification within and across eye fixations. Journal of Experimental Psychology: Human Perception and Performance, 20, 826-839.

Henderson, J. M., Dixon, P., Petersen, A., Twilley, L. C., \& Ferreira, F. (1995). Evidence for the use of phonological representations during transsaccadic word recognition. Journal of Experimental Psychology: Human Perception and Performance, 21, 82-97.

Henderson, J. M., \& Ferreira, F. (1990). Effects of foveal processing difficulty on the perceptual span in reading: Implications for attention and eye movement control. Journal of Experimental Psychology: Learning, Memory, and Cognition, 16, 417-429.

Henderson, J. M., \& Ferreira, F. (1993). Eye movement control during reading: Fixation measures reflect foveal but not parafoveal processing difficulty. Caradian Journal of Experimental Psychology, 47, 201-221.

Henderson, J. M., \& Hollingworth, A. (1998). Eye movements during scene viewing: An overview. In G. Underwood (Ed.), Eye guidance in reading and scene perception (pp. 269-294). Oxford, England: Elsevier.

Henderson, J. M., McClure, K. K., Pierce, S., \& Schrock, G. (1997). Object identification without foveal vision: Evidence from an artificial scotoma paradigm. Penception \& Psychophysics, 59, 323-346.

Henderson, J. M., Pollatsek, A., \& Rayner, K. (1987). The effects of foveal priming and extrafoveal preview on object identification. Journal of Experimental Psychology: Human Perception and Performance, 13, 449-463.

Henderson, J. M., Pollatsek, A., \& Rayner, K. (1989). Covert visual attention and extrafoveal information use during object identification. Perception \& Psychophysics, 45, 196-208.

Henderson, J. M., \& Siefert, A. B. C. (in press-a). The influence of enantiomorphic transformation on transsaccadic object integration. 
Journal of Experimental Psychology: Human Perception and Performance.

Henderson, J, M., \& Siefert, A. B. D. (in press-b). Types and tokens in transsaccadic object identification: Effects of spatial position and leftright orientation. Psychonomic Bulletin \& Review.

Henderson, J. M., Weeks, P. A., \& Hollingworth, A. (in press). The effects of semantic consistency on eye movements during complex scene viewing. Journal of Experimental Psychology: Human Perception and Performance.

Henriks, A. W. (1996). Vergence eye movements during fixations in reading. Acta Psychologica, 92, 131-151.

Heywood, S., \& Churcher, J. (1980). Structure of the visual array and saccadic latency: Implications for oculomotor control. Quarterly Journal of Experimental Psychology, 32, 335-341.

Hoffman, J. E., \& Subramaniam, B. (1995). The role of visual attention in saccadic eye movements. Perception \& Psychophysics, 57, 787795.

Hogaboam, T. W. (1983). Reading patterns in eye movements. In $\mathrm{K}$. Rayner (Ed.), Eye movements in reading (pp. 309-332). New York: Academic Press.

Hogaboam, T. W., \& McConkie, G. W. (1981). The rocky road from eye fixations to comprehension (Tech. Rep. No. 207). University of Illinois at Urbana-Champaign, Center for the Study of Reading.

Holmes, V. M., \& O'Regan, J. K. (1981). Eye fixation patterns during the reading of relative-clause sentences. Journal of Verbal Learning and Verbal Behavior, 20, 417-430.

Holmes, V. M., \& O'Regan, J. K. (1987). Decomposing French words. In J. K. O'Regan \& A. Lévy-Schoen (Eds.), Eye movements: From physiology to cognition (pp. 459-466). Amsterdam: North Holland.

Holmes, V. M., \& O'Regan, J. K. (1992). Reading derivationally affixed French words. Language and Cognitive Processes, 7, 163-192.

Hooge, I. T. C., \& Erkelens, C. J. (1996). Control of fixation duration during a simple search task. Perception \& Psychophysics, 58, 969976.

Hooge, I. T. C., \& Erkelens, C. J. (1998). Adjustment of fixation duration during visual search. Vision Research, 38, 1295-1302.

Huey, E. B. (1908). The psychology and pedagogy of reading. New York: Macmillan.

Hyönä, J. (1993). Effects of thematic and lexical priming on readers' eye movements. Scandinavian Journal of Psychology, 34, 293-304.

Hyönä, J. (1995a). Do irregular letter combinations attract readers' attention? Evidence from fixation locations in words. Journal of Experimental Psychology: Human Perception and Performance, 21, 6881.

Hyönä, J. (1995b). An eye movement analysis of topic-shift effect during repeated reading. Journal of Experimental Psychology: Learning, Memory, and Cognition, 21, 1365-1373.

Hyönä, J., \& Hujanen, H. (1997). Effects of case marking and word order on sentence parsing in Finnish: An eye fixation analysis. Quarterly Journal of Experimental Psychology, 50A, 841-858.

Hyönä, J., \& Jarvella, R. J. (1993). Time course of context effects during reading: An eye fixation analysis. In G. d'Ydewalle \& J. Van Rensbergen (Eds.), Perception and cognition: Advances in eye movement research (pp. 239-249). Amsterdam: North Holland.

Hyönä, J., \& Niemi, P. (1990). Eye movements in repeated reading of a text. Acta Psychologica, 73, 259-280.

Hyönä, J., Niemi, P., \& Underwood, G. (1989). Reading long words embedded in sentences: Informativeness of word halves affects eye movements. Journal of Experimental Psychology: Human Perception and Performance, 15, 142-152.

Hyönä, J., \& Olson, R. K. (1995). Eye movement patterns among dyslexic and normal readers: Effects of word length and word frequency. Journal of Experimental Psychology: Learning, Memory, and Cognition, 21, 1430-1440.

Hyönä, J., \& Pollatsek, A. (in press). Reading Finnish compound words:
Eye fixations are affected by component morphemes. Journal of Experimental Psychology: Human Perception and Performance.

Ikeda, M., \& Saida, S. (1978). Span of recognition in reading. Vision Research, 18, 83-88.

Ikeda, M., Saida, S., \& Sugiyama, T. (1977). Visual field size necessary for length comparison. Perception \& Psychophysics, 22, 165-170.

Inhoff, A. W. (1984). Two stages of word processing during eye fixations in the reading of prose. Journal of Verbal Learning and Verbal Behavior, 23, 612-624

Inhoff, A.W. (1985). The effect of factivity on lexical retrieval and postlexical processing during eye fixations in reading. Journal of Psycholinguistic Research, 14, 45-56.

Inhoff, A. W. (1986). Preparing sequences of saccades. Acta Psychologica, 61, 211-228.

Inhoff, A. W. (1987). Parafoveal word perception during eye fixations in reading: Effects of visual salience and word structure. In $\mathrm{M}$. Coltheart (Ed.), Attention and performance (Vol. 12, pp. 403-420). London: Erlbaum.

Inhoff, A. W. (1989a). Lexical access during eye fixations in reading: Are word access codes used to integrate lexical information across interword fixations? Journal of Memory and Language, 28, 444-461.

Inhoff, A. W. (1989b). Parafoveal processing of words and saccade computation during eye fixations in reading. Journal of Experimental Psychology: Human Perception and Performance, 15, 544-555.

Inhoff, A. W. (1990). Integrating information across eye fixations in reading: The role of letter and word units. Acta Psychologica, 73, 281-297.

Inhoff, A. W. (1991). Effects of word-frequency during copytyping. Journal of Experimental Psychology: Human Perception and Performance, 17, 478-488.

Inhoff, A. W., Bohemier, G., \& Briihl, D. (1992). Integrating text across fixations in reading and copytyping. In $\mathrm{K}$. Rayner (Ed.), Eye movements and visual cognition: Scene perception and reading (pp. $355-$ 368). New York: Springer-Verlag.

Inhoff, A. W., \& Briihl, D. (1991). Semantic processing of unattended text during selective reading: How the eyes see it. Perception \& Psychophysics, 49, 289-294.

Inhoff, A. W., Briihl, D., Bohemier, G., \& Wang, J. (1992). Eye-hand span and coding of text during copytyping. Journal of Experimental Psychology: Learning, Memory, and Cognition, 18, 298-306.

Inhoff, A. W., Briihl, D., \& Schwartz, J. (1996). Compound word effects differ in reading, on-line naming, and delayed naming tasks. Memory \& Cognition, $24,466-476$.

Inhoff, A. W., Briihl, D., \& Starr, M. (1998). Parafovea-to-fovea priming during eye fixations in reading. Manuscript submitted for publication.

Inhoff, A. W., Calabrese, J., \& Morris, R. K. (1986). Eye movements in skilled typing. Bulletin of the Psychonomic Society, 24, 113-114.

Inhoff, A. W., Chiu, T., \& Wang, J. (1990). Coordination of eye and finger movements in copytyping. Bulletin of the Psychonomic Society, $28,302-304$.

Inhoff, A. W., \& Gordon, A. M. ( 1997 ). Eye movements and eye-hand coordination during typing. Current Directions in Psychological Science, $6,153-157$.

Inhoff, A. W., Lima, S. D., \& Carroll, P. J. (1984). Contextual effects on metaphor comprehension in reading. Memory \& Cognition, 12, $558-567$.

Inhoff, A. W., \& Liu, W. (1998). The perceptual span and oculomotor activity during the reading of Chinese sentences. Journal of Experimental Psychology: Human Perception and Performance, 24, 20-34. Inhoff, A. W., Pollatsek, A., Posner, M. I., \& Rayner, K. (1989). Covert attention and eye movements during reading. Quarterly Journal of Experimental Psychology, 41A, 63-89.

Inhoff, A. W., \& Radach, R. (1998). Definition and computation of 
oculomotor measures in the study of cognitive processes. In G. Underwood (Ed.), Eye guidance in reading and scene perception (pp. 29-54). Oxford, England: Elsevier.

Inhoff, A. W., Radach, R., \& Heller, D. (1996). Morphological processes during word recognition. Abstracts of the Psychonomic Society, 1, 54.

Inhoff, A. W., \& Rayner, K. (1986). Parafoveal word processing during eye fixations in reading: Effects of word frequency. Perception \& Psychophysics, 40, 431-439.

Inhoff, A. W., Starr, M., Liu, W., \& Wang, J. (1998). Eye-movementcontingent display changes are not compromised by flicker and phosphor persistence. Psychonomic Bulletin \& Review, 5, 101-106.

Inhoff, A. W., \& Topolski, R. (1992). Lack of semantic activation from unattended text during passage reading. Bulletin of the Psychonomic Society, 30, 365-366.

Inhoff, A. W., \& Topolski, R. (1994). Use of phonological codes during eye fixations in reading and in on-line and delayed naming tasks. Journal of Memory and Language, 33, 689-713.

Inhoff, A. W., Topolski, R., Vitu, F., \& O'Regan, J. K. (1993). Attention demands during reading and the occurrence of brief (express) fixations. Perception \& Psychophysics, 54, 814-823.

Inhoff, A. W., Topolski, R., \& Wang, J. (1992). Saccade programming during short duration fixations: An examination of copytyping, letter detection, and reading. Acta Psychologica, 81, 1-21.

Inhoff, A. W., \& Tousman, S. (1991). Lexical integration across saccades in reading. Psychological Research, 51, 330-337.

Inhoff, A. W., \& Wang, J. (1992). Encoding of text, manual movement planning, and eye-hand coordination during copytyping. Journal of Experimental Psychology: Human Perception and Performance, 18, 437-448.

Irwin, D. E. (1991) . Information integration across saccadic eye movements. Cognitive Psychology, 23, 420-456.

Irwin, D. E. (1992a). Memory for position and identity across eye movements. Journal of Experimental Psychology: Learning, Memory, and Cognition, 18, 307-317.

Irwin, D. E. (1992b). Visual memory within and across fixations. In K. Rayner (Ed.), Eye movements and visual cognition: Scene perception and reading (pp. 146-165). New York: Springer-Verlag.

Irwin, D. E. (1993). Perceiving an integrated world. In D. E. Meyer \& S. Kornblum (Eds.), Attention and performance (Vol. 14, pp. 121 142). Cambridge, MA: MIT Press.

Irwin, D. E. (1994). On the measurement of phosphor persistence in oscilloscopic displays. Vision Research, 34, 1623.

Irwin, D. E. (1996). Integrating information across saccadic eye movements. Current Directions in Psychological Science, 5, 94-100.

Irwin, D. E. (1998) . Lexical processing during saccadic eye movements. Cognitive Psychology, 36, 1-27.

Irwin, D. E., \& Andrews, R. (1996). Integration and accumulation of information across saccadic eye movements. In T. Inui \& J. McClelland (Eds.), Attention and performance (Vol. 16, pp. 125-155). Cambridge, MA: MIT Press.

Irwin, D. E., Brown, J. S., \& Sun, J. (1988). Visual masking and visual integration across saccadic eye movements. Joumal of Experimental Psychology: General, 117, 276-287.

Irwin, D. E., \& Carlson-Radvansky, L. A. (1996). Cognitive suppression during saccadic eye movements. Psychological Science, 7, 8388.

Irwin, D. E., Carlson-Radvansky, L. A., \& Andrews, R. V. (1995). Information processing during saccadic eye movements. Acta Psychologica, 90, 261-273.

Irwin, D. E., \& Gordon, R. D. (1998). Eye movements, attention, and transsaccadic memory. Visual Cognition, 5, 127-156.

Irwin, D. E., McConkie, G. W., Carlson-Radvansky, L., \& Currie, C.
(1994). A localist solution for visual stability across saccades. Behavioral and Brain Sciences, 17, 265-266.

Irwin, D. E., Yantis, S., \& Jonides, J. (1983). Evidence against visual integration across saccadic eye movements. Perception \& Psychophysics, 34, 49-57.

Irwin, D. E., Zacks, J. L., \& Brown, J. S. (1990). Visual memory and the perception of a stable visual environment. Perception \& Psychophysics, 47, 35-46.

Ishida, T., \& Ikeda, M. (1989). Temporal properties of information extraction in reading studied by a text-mask replacement technique. Journal of the Optical Society A: Optics and Image Science, 6, 16241632.

Jacob, R. J. K. (1991). The use of eye movements in human-computer interaction techniques: What you look at is what you get. ACM Trans actions on Information Systems, 9, 152-169.

Jacobs, A. M. (1986). Eye movement control in visual search: How direct is visual span control? Penception \& Psychophysics, 39, 4758.

Jacobs, A. M. (1987a). On localization and saccade programming. $V i$ sion Research, 27, 1953-1966.

Jacobs, A. M. (1987b). On the role of blank spaces for eye-movement control in visual search. Perception \& Psychophysics, 41, 473-479.

Jacobs, A. M. (1987c). Toward a model of eye movement control in visual search. In J. K. O'Regan \& A. Lévy-Schoen (Eds.), Eye movements: From physiology to cognition (pp. 275-284). Amsterdam: North Holland.

Jacobs, A. M. (1991). The search operating characteristic as a tool for analyzing performance in dynamic visual search tasks. Spatial Vision, $5,269-277$.

Jacobs, A. M., Nazir, T. A., \& Heller, O. (1989). Perception of lower case letters in peripheral vision: A discrimination matrix based on saccade latencies. Perception \& Psychophysics, 46, 95-102.

Jacobs, A. M., \& O'Regan, J. K. (1987). Spatial and/or temporal adjustments of scanning behavior to visibility changes. Acta Psychologica, $65,133-146$.

Jacobson, J. Z., \& Dodwell, P. C. (1979). Saccadic eye movements during reading. Brain and Language, 8, 303-314.

Jared, D., Levy, B. A., \& Rayner, K. (in press). The role of phonology in the activation of word meanings during reading: Evidence from proofreading and eye movements. Journal of Experimental Psychology: General.

Jonides, J., Irwin, D. E., \& Yantis, S. (1982). Integrating visual information from successive fixations. Science, 215, 192-194.

Jonides, J., Irwin, D. E., \& Yantis, S. (1983). Failure to integrate information from successive fixations. Science, 222, 188.

Jordan, T. R., Patching, G. R., \& Milner, A. D. (1998). Ensuring central fixations in divided visual field studies of cerebral asymmetry: Instructions are not enough. Quarterly Journal of Experimental Psychology, 51A, 371-392.

Juola, J. F., Ward, N. J., \& McNamara, T. (1982). Visual search and reading of rapid serial visual presentations of letter strings, words, and text. Journal of Experimental Psychology: General, 111, 208227.

Just, M. A., \& Carpenter, P. A. (1978). Inference processing during reading: Reflections from eye fixations. In J.W. Senders, D. F. Fisher, \& R. A. Monty (Eds.), Eye movements and the higher psychological functions (pp. 157-174). Hillsdale, NJ: Erlbaum.

Just, M. A., \& Carpenter, P. A. (1980). A theory of reading: From eye fixations to comprehension. Psychological Review, 87, 329-354.

Just, M. A, \& Carpenter, P. A. (1985). Cognitive coordinate systems: Accounts of mental rotation and individual differences in spatial ability. Psychological Review, 92, 137-172.

Just, M. A., \& Carpenter, P. A. (1987). The psychology of reading and language comprehension. Boston: Allyn \& Bacon. 
Just, M. A., Carpenter, P. A., \& Masson, M. E. J. (1982). What eye fwations tell us about speed reading and skimming. (Eye-lab Technical Report). Carnegie-Mellon University, Pittsburgh, PA.

Just, M. A., Carpenter, P. A., \& Woolley, J. D. (1982). Paradigms and processes in reading comprehension. Journal of Experimental Psy. chology: General, 111, 228-238.

Juttner, M. (1997). Effects of perceptual context on transsaccadic visual matching. Perception \& Psychophysics, 59, 762-773.

Juttner, M., \& Rohler, R. (1993). Lateral information transfer across saccadic eye movements. Perception \& Psychophysics, 53, 210-220.

Kalesnykas, R. P., \& Hallett, P. E. (1995). Retinal eccentricity and the latency of eye saccades. Vision Research, 34, 517-531.

Kapoula, Z: A. (1983). The influence of peripheral preprocessing on oculomotor programming in a scanning task. In R. Groner, C. Menz, D. F. Fisher, \& R. A. Monty (Eds.), Eye movements and psychological functions: International views (pp. 101-114). Hillsdale, NJ: Erlbaum.

Kapoula, Z. A. (1984). Comment les yeux explorentails des alignments graphiques? [How do the eyes explore lines of symbols?] Annee Psychologique, 84, 207-226.

Kapoula, Z. A. (1985). Evidence for a range effect in the visual system. Vision Research, 25, 1155-1157.

Kapoula, Z. A., \& Robinson, D. A. (1986). Saccadic undershoot is not inevitable: Saccades can be accurate. Vision Research, 26, 735-743.

Karnath, H. O. (1994). Spatial limitations of eye movements during ocular exploration of simple line drawings in neglect syndrome. Cortex, 30, 319-330.

Karnath, H. O., \& Huber, W. (1992). Abnormal eye movement behavior during text reading in neglect syndrome: A case study. Neuropsychologia, 30, 593-598.

Katsanis, J., Kortenkamp, S., Iacono, W. G., \& Grove, W. M. (1997). Antisaccade performance in patients with schizophrenia and affective disorder. Journal of Abnormal Psychology, 106, 468-472.

Kawabata, N., Yamagami, K., \& Noaki, M. (1978). Visual fixation points and depth perception. Vision Research, 18, 853-854.

Kennedy, A. (1983). On looking into space. In K. Rayner (Ed.), Eye movements in reading: Perceptual and language processes (pp. 237251). New York: Academic Press.

Kennedy, A. (1987). Eye movements, reading skill and the spatial code. In J. Beech \& A. Colley (Eds.), Cognitive approaches to reading (pp. 169-186). Chichester, England: Wiley.

Kennedy, A. (1992). The spatial coding hypothesis. In K. Rayner (Ed.), Eye movements and visual cognition: Scene perception and reading (pp. 379-396). New York: Springer-Verlag.

Kennedy, A. (1998). The influence of parafoveal words on foveal inspection time: Evidence for a processing tradeoff. In G. Underwood (Ed.), Eye guidance in reading and scene perception (pp. 149-180). Oxford, England: Elsevier.

Kennedy, A., \& Baccino, T. (1995). The effects of screen refresh rate on editing operations using a computer mouse pointing device. Quarterly Journal of Experimental Psychology, 48A, 55-71.

Kennedy, A., Brysbaert, M., \& Murray, W. S. (1998). The effects of intermittent illumination of a visual inspection task. Quarterly Journal of Experimental Psychology, 51A, 135-152.

Kennedy, A., \& Murray, W. S. (1984). Inspection times for words in syntactically ambiguous sentences under three presentation conditions. Journal of Experimental Psychology: Human Perception and Performance, 10, 833-849.

Kennedy, A., \& Murray, W. S. (1987a). The components of reading time: Eye movement patterns of good and poor readers. In J. K. O'Regan \& A. Lévy-Schoen (Eds.), Eye movements: From physiology to cognition (pp. 509-520). Amsterdam: North Holland.

Kennedy, A., \& Murray, W. S. (1987b). Spatial coordinates and reading:
Comments on Monk. Quarterly Joumal of Experimental Psychology, $39 A, 649-656$.

Kennedy, A., \& Murray, W. S. (1991). The effects of flicker on eye movement control. Quarterly Journal of Experimental Psychology, 43A, 79-99.

Kennedy, A., \& Murray, W. S. (1993). 'Flicker' on VDU screens. $\mathrm{Na}$ ture, $365,213$.

Kennedy, A., \& Murray, W. S. (1996). Eye movement control during the inspection of words under conditions of pulsating illumination. European Journal of Cognitive Psychology, 8, 381-404.

Kennedy, A., Murray, W. S., Jennings, F., \& Reid, C. (1989). Parsing complements: Comments on the generality of the principle of minimal attachment [Special issue]: Language and Cognitive Processes, 4, $51-76$.

Kennedy, A., \& Pidcock, B. (1981). Eye movements and variations in reading time. Psychological Research, 43, 69-79.

Kennison, S. M., \& Clifton, C. (1995). Determinants of parafoveal preview benefit in high and low working memory capacity readers: Implications for eye movement control. Journal of Experimental Psychology: Learning, Memory, and Cognition, 21, 68-81.

Kennison, S. M., \& Gordon, P. C. (1998). Comprehending referential expressions during reading: Evidence from eye tracking. Discourse Processes, 24, 229-252.

Kentridge, R. W., Heywood, C. A., \& Weiskrantz, L. (1997). Residual vision in multiple retinal locations within a scotoma: Implications for blindsight. Journal of Cognitive Neuroscience, 9, 191-202.

Kerr, J. S., \& Underwood, G. (1984). Fixation time on anaphoric pronouns decreases with contiguity of reference. In A. G. Gale \& F. Johnson (Eds.), Theoretical and applied aspects of eye movement research (pp. 195-202). Amsterdam: North Holland.

Kingstone, A., \& Klein, R. M. (1993a). Visual offset facilitates saccade latency: Does pre-disengagement of attention mediate the gap effect? Journal of Experimental Psychology: Human Perception and Performance, 19, 251-265.

Kingstone, A., \& Klein, R. M. (1993b). What are human express saccades? Perception \& Psychophysics, 54, 260-273.

Kinsier, V., \& Carpenter, R. H. S. (1995). Saccadic eye movements while reading music. Vision Research, 35, 1447-1458.

Klein, R. M. (1980). Does oculomotor readiness mediate cognitive control of visual attention? In R. Nickerson (Ed.), Attention and performance. (Vol. 8, pp. 259-276). Hillsdale, NJ: Erlbaum.

Klein, R., Berry, G., Briand, K., D'Entremont, B., \& Farmer, M. (1990). Letter identification declines with increasing retinal eccentricity at the same rate for normal and dyslexic readers. Perception \& Psychophysics, 47, 601-606.

Klein, R., \& Farrell, M. (1989). Search performance without eye movements. Perception \& Psychophysics, 46, 476-482.

Klein, R. M., Kingstone, A., \& Pontefract, A. (1992). Orienting of visual attention. In K. Rayner (Ed.), Eye movements and visual cognition: Scene perception and reading (pp. 46-65). New York: SpringerVerlag.

Kliegl, R., \& Olson, R. K. (1981). Reduction and calibration of eye movement data. Behavior Research Methods and Instrumentation, 13, $107-111$.

Kliegl, R., Olson, R. K., \& Davidson, B. J. ( 1982). Regression analyses as a tool for studying reading processes: Comments on Just and Carpenter's eye fixation theory. Memory \& Cognition, 10, 287-296.

Kohsom, C., \& Gobet, F. (1997). Adding spaces to Thai and English: Effects on reading. Proceedings of the Cognitive Science Society, 19, 388-393.

Kolers, P. A. (1976). Buswell's discoveries. In R. A. Monty \& J. W. Senders (Eds.), Eye movements and psychological processes (pp. 371-395). Hillsdale, NJ: Erlbaum.

Kolers, P. A., Duchnicky, R. L., \& Ferguson, D. C. (1981). Eye move- 
ment measurement of readability of CRT displays. Human Factors, $23,517-527$.

Konieczny, L., Hemforth, B., Scheepers, C., \& Strube, G. (1997). The role of lexical heads in parsing: Evidence from German. Language and Cognitive Processes, 12, 307-348.

Kowler, E., Anderson, E., Dosher, B., \& Blaser, E. (1995). The role of attention in the programming of saccades. Vision Research, 35, 18971916.

Kowler, E., \& Anton, S. (1987). Reading twisted text: Implications for the role of saccades. Vision Research, 27, 45-60.

Kowler, E., \& Blaser, E. (1995). The accuracy and precision of saccades to small and larger targets. Vision Research, 35, 1741-1754.

Kowler, E., \& Martins, A. J. (1985). Eye movements of preschool children. Science, 215, 997-999.

Kowler, E., \& Steinman, R. M. (1977). The role of small saccades in counting. Vision Research, 17, 141-146.

Kowler, E., \& Steinman, R. M. (1979). Miniature saccades: Eye movements that do not count. Vision Research, 19, 105-108.

Kristjanson, A. F., \& Antes, J. R. (1989). Eye movement analysis of artists and nonartists viewing paintings. Visual Arts Research, 15, 2130.

Ladavas, E., Zeloni, G., Zaccara, G., \& Gangemi, P. (1997). Eye movements and orienting of attention in patients with visual neglect. Journal of Cognitive Neumscience, 9, 67-74.

Land, M. F. (1992). Predictable eye-head coordination during driving. Nature, 359, 318-320.

Land, M. F., \& Furneaux, S. (1997). The knowledge base of the oculomotor system. Philosophical Transactions of the Royal Society of London B, 352, 1231-1239.

Land, M. F., \& Horwood, J. (1995). Which part of the road guides steering? Nature, 377, 339-340.

Land, M. F., \& Lee, D. N. (1994). Where we look when we steer. Nature, 369, 742-744.

Lansing, C. R., \& McConkie, G. W. (1994). A new method for speech reading research: Tracking observers' eye movements. Journal of the Academy of Rehabilitation Audiology, 27, 25-43.

Larsen, S., \& Parlenvi, P. (1984). Patterns of inverted reading and subgroups in dyslexia. Annals of Dyslexia, 34, 195-203.

Lee, Y., Binder, K. S., Kim, J., Pollatsek, A., \& Rayner, K. (in press). Activation of phonological codes during eye fixations in reading. Journal of Experimental Psychology: Human Perception and Performance.

Lefton, L. A., Nagle, R. J., Johnson, G., \& Fisher, D. F. (1979). Eye movement dynamics of good and poor readers: Then and now. Journal of Reading Behavior, 11, 319-328.

Legge, G. E., Klitz, T. S., \& Tjan, B. S. (1997). Mr. Chips: An idealobserver model of reading. Psychological Review, 104, 524-553.

Legge, G. E., Rubin, G. S., Pelli, D. G., \& Schleske, M. M. (1985). Psychophysics of reading: II. Low vision. Vision Research, 25, 253266.

Leigh, R. J., \& Zee, D. S. (1991). The neurology of eye movements. Philadelphia: F. A. Davis.

Lennerstrand, G., Ygge, J., \& Rydberg, A. (1994). Binocular control in normally reading children and dyslexics. In J. Ygge \& G. Lennerstrand (Eds.), Eye movements in reading (pp. 291-300). Oxford, England: Pergamon Press.

Lesch, M. F., \& Pollatsek, A. (1993). Automatic activation of semantic information by phonological codes in visual word recognition. Journal of Experimental Psychology: Learning, Memory, and Cognition, $19,285-294$.

Lesch, M. F., \& Pollatsek, A. (1998). Evidence for the use of assembied phonology in accessing the meaning of printed words. Journal of Experimental Psychology: Learning, Memory, and Cognition, 24, 573-592.
Lévy-Schoen, A. (1981). Flexible and/or rigid control of oculomotor scanning behavior. In D. F. Fisher, R. A. Monty, \& J. W. Senders (Eds.), Eye movements: Cognition and visual perception (pp. 299 316). Hillsdale, NJ: Erlbaum.

Lévy-Schoen, A., \& O'Regan, K. (1979). The control of eye movements in reading. In P. A. Kolers, M. E. Wrolstad, \& H. Bouma (Eds.), Processing of visible language (pp. 7-36). New York: Plenum.

Lima, S. D. (1987). Morphological analysis in sentence reading. Journal of Memory and Language, 26, 84-99.

Lima, S. D., \& Inhoff, A. W. (1985). Lexical access during eye fixations in reading: Effects of word-initial letter sequences. Joumal of Experimental Psychology: Human Perception and Performance, 11, 272285.

Liu, A. (1998). What the driver's eye tells the car's brain. In G. Underwood (Ed.), Eye guidance in reading and scene perception (pp. 431-452). Oxford, England: Elsevier.

Liversedge, S. P., Paterson, K. B., \& Pickering, M. J. (1998). Eye movements and measures of reading time. In G. Underwood (Ed.), Eye movements in reading and scene perception (pp. 55-76). Oxford, England: Elsevier.

Liversedge, S. P., Pickering, M. J., Branigan, H. P., \& van Gompel, R.P. G. (1998). Processing arguments and adjuncts in isolation and context: The case of by-phrase ambiguities in passives. Journal of Experimental Psychology: Learning, Memory, and Cognition, 24, 461-475.

Liversedge, S. P., \& Underwood, G. (1998). Foveal processing load and landing position effects in reading. In G. Underwood (Ed.), Eye guidance in reading and scene perception (pp. 201-222). Oxford, England: Elsevier.

Locher, P. J., \& Nodine, C. G. (1974). The role of scanpaths in the recognition of random shapes. Perception \& Psychophysics, 15, 308314.

Loftus, E. F, Loftus, G. R., \& Messo, J. (1987). Some facts about "Weapon Focus." Law and Human Behavior, 11, 55-62.

Loftus, G. R. (1972). Eye fixations and recognition memory for pictures. Cognitive Psychology, 3, 525-551.

Loftus, G. R. (1981). Tachistoscopic simulations of eye fixations on pictures. Journal of Experimental Psychology: Human Learning and Memory, 7, 369-376.

Loftus, G. R. (1983). Eye fixations on text and scenes. In K. Rayner (Ed.), Eye movements in reading: Perceptual and language processes (pp. 359-376). New York: Academic Press.

Loftus, G. R. (1985). Picture perception: Effects of luminance on available information and information-extraction rate. Journal of Experimental Psychology: General, 114, 342-356.

Loftus, G. R., Kaufman, L., Nishimoto, T., \& Ruthruff, E. (1992). Effects of visual degradation on eye-fixation durations, perceptual processing, and long-term visual memory. In K. Rayner (Ed.), Eye movements and visual cognition: Scene perception and reading (pp. 203226). New York: Springer.

Loftus, G. R., \& Mackworth, N. H. (1978). Cognitive determinants of fixation location during picture viewing. Journal of Experimental Psychology: Human Perception and Performance, 4, 565-572.

Luria, S. M., \& Strauss, M. S. (1978). Comparison of eye movements over faces in photographic positives and negatives. Perception, 7 , 349-358.

Mackworth, N. H., \& Morandi, A. J. (1967). The gaze selects informative details within pictures. Perception and Psychophysics, 2, $547-$ 552.

Magliano, J. P., Graesser, A. C., Eymard, L. A., Haberlandt, K., \& Gholson, B. (1993). Locus of interpretive and inference processes during text comprehension: A comparison of gaze durations and word reading times. Journal of Experimental Psychology: Learning, Memory, and Cognition, 19, 704-709. 
Mannen, S. K., Ruddock, K. H., \& Wooding, D. S. (1995). Automatic control of saccadic eye movements made in visual inspection of briefly presented 2-D images. Spatial Vision, 9, 363-386.

Mannen, S. K., Ruddock, K. H., \& Wooding, D. S. (1996). The relationship between the locations of spatial features and those of fixation made during visual examination of briefly presented images. Spatial Vision, 10, 165-188.

Mannen, S. K., Ruddock, K. H., \& Wooding, D. S. (1997). Fixation patterns made during brief examination of two-dimensional images. Perception, 26, 1059-1072.

Martos, F. J., \& Vila, J. (1990). Differences in eye movement control among dyslexic, retarded and normal readers in the Spanish population. Reading and Writing, 2, 175-188.

Masson, M. E. J. (1983). Conceptual processing of text during skimming and rapid sequential reading. Memory \& Cognition, 11, 262274.

Matin, E. (1974). Saccadic suppression: A review. Psychological Bulletin, 81, 899-917.

Matin, E., Shao, K., \& Boff, K. (1993). Saccadic overhead: Information processing time with and without saccades. Perception \& Psychophysics, 53, 372-380.

May, J. G., Kennedy, R. S., Williams, M. C., Dunlop, W. P., \& Brannan, J. R. (1990). Eye movement indices of mental workload. Acta Psychologica, 75, 75-89.

Mazuka, R., Itoh, K., \& Kondo, T. (1997). Processing down the garden path in Japanese: Processing of sentences with lexical homonyms. Journal of Psycholinguistic Research, 26, 207-228.

McClelland, J. L., \& O'Regan, J. K. (1981). Expectations increase the benefit derived from parafoveal visual information in reading words aloud. Joumal of Experimental Psychology: Human Perception and Performance, 7, 634-644.

McConkie, G. W. (1979). On the role and control of eye movements in reading. In P. A. Kolers, M. E. Wrolstad, \& H. Bouma (Eds.), Processing of visible language (pp. 37-48). New York: Plenum.

McConkie, G. W. (1981). Evaluating and reporting data quality in eye movement research. Behavior Research Methods \& Instrumentation, 13, 97-106.

McConkie, G. W. (1997). Eye movement contingent display control: Personal reflections and comments. Scientific Studies of Reading, 1 , 303-316.

McConkie, G. W., \& Currie, C. B. (1996). Visual stability across saccades while viewing complex pictures. Journal of Experimental Psychology: Human Perception and Performance, 22, 563-581.

McConkie, G. W., \& Hogaboam, T. W. (1985). Eye position and word identification in reading. In R. Groner, G. W. McConkie, \& C. Menz (Eds.), Eye movements and human information processing (pp. 159172). Amsterdam: North Holland.

McConkie, G. W., Hogaboam, T. W., Wolverton, G. S., Zola, D. W., \& Lucas, P. A. (1979). Toward the use of eye movements in the study of language processing. Discourse Processes, 2, 157-177.

McConkie, G. W., Kerr, P. W., \& Dyre, B. P. (1994). What are "normal" eye movements during reading: Toward a mathematical description. In J. Ygge \& G. Lennerstrand (Eds.), Eye movements in reading (pp. 315-328). Oxford, England: Pergamon Press.

McConkie, G. W., Kert, P. W., Reddix, M. D., \& Zola, D. (1988). Eye movement control during reading: I. The location of initial eye fixations in words. Vision Research, 28, 1107-1118.

McConkie, G. W., Kerr, P. W., Reddix, M. D., Zola, D., \& Jacobs, A. M. (1989). Eye movement control during reading: II. Frequency of refixating a word. Perception \& Psychophysics, 46, 245-253.

McConkie, G. W., \& Rayner, K. (1975). The span of the effective stimulus during a fixation in reading. Perception \& Psychophysics, 17. $578-586$.
McConkie, G. W., \& Rayner, K. (1976a). Asymmetry of the perceptual span in reading. Bulletin of the Psychonomic Society, 8, 365-368.

McConkie, G. W., \& Rayner, K. (1976b). Identifying the span of the effective stimulus of reading: Literature review and theories of reading. In H. Singer \& R. B. Ruddell (Eds.), Theoretical models and processes in reading (pp. 137-162). Newark, DE: International Reading Association.

McConkie, G. W., Reddix, M. D., \& Zola, D. (1992). Perception and cognition in reading: Where is the meeting point? In K. Rayner (Ed.), Eye movements and visual cognition: Scene perception and reading (pp. 293-303). New York: Springer-Verlag.

McConkie, G. W., Underwood, N. R., Wolverton, G. S., \& Zola, D. (1988). Some properties of eye movement control during reading. In G. Luer, U. Lass, \& J. Shallo-Hoffman (Eds.), Eye movement reseanch: Physiological and psychological aspects (pp. 226-245). Toronto: C. J. Hogrefe.

McConkie, G. W., Wolverton, G. S., \& Zola, D. (1984). Instrumentation considerations in research involving eye-movement contingent stimulus control. In A. G. Gale \& F. Johnson (Eds.), Theoretical and applied aspects of eye movement research (pp. 39-47). Amsterdam: North Holland.

McConkie, G. W., Wolverton, G. S., Zola, D., \& Burns, D. B. (1978). Eye movement contingent display control in studying reading. Behav. ior Research Methods \& Instruments, 10, 154-166.

McConkie, G. W., \& Zola, D. (1979). Is visual information integrated across successive fixations in reading? Perception \& Psychophysics, 25, 221-224.

McConkie, G. W., \& Zola, D. (1984). Eye movement control during reading: The effect of words units. In W. Prinz \& A. F. Sanders (Eds.), Cognition and motor processes (pp. 63-74). Berlin: Springer-Verlag.

McConkie, G. W., \& Zola, D., (1987). Visual attention during eye fixations while reading. In M. Coltheart (Ed.), Attention and performance (Vol. 12, pp. 385-401). London: Erlbaum.

McConkie, G. W., Zola, D., \& Blanchard, H. E. (1984). What is the basis for making an eye movement during reading? In A. G. Gale \& F. Johnson (Eds.), Theoretical and applied aspects of eye movement research (pp. 169-178). Amsterdam: North Holland.

McConkie, G. W., Zola, D., Blanchard, H. E., \& Wolverton, G. S. (1982). Perceiving words during reading: Lack of facilitation from prior peripheral exposure. Perception \& Psychophysics, 32, 271-282.

McConkie, G. W., Zola, D., Grimes, J., Kerr, P. W., Bryant, N. R., \& Wolff, P. M. (1991). Children's eye movements during reading. In J. F. Stein (Ed.), Vision and visual dyslexia (pp. 251-262). London: Macmillan Press.

McConkie, G. W., Zola, D., \& Wolverton, G. S. (1980, April). How precise is eye guidance in reading? Paper presented at the meeting of the American Educational Research Association, Boston.

McConkie, G. W., Zola, D., \& Wolverton, G. S. (1985). Estimating frequency and size of effects due to experimental manipulations in eye movement research. In R. Groner, G. W. McConkie, \& C. Menz (Eds.), Eye movements and human information processing (pp. 137148). Amsterdam: North Holland.

McDowell, E. D., \& Rockwell, T. H. (1978). An exploratory investigation of the stochastic nature of the drivers' eye movements and their relationship to the roadway geometry. In J. W. Senders, D. F. Fisher, \& R. A. Monty (Eds.), Eye movements and the higher psychological functions (pp. 329-345). Hillsdale, NJ: Erlbaum.

McGowan, J. W., Kowler, E., Sharma, A., \& Chubb, C. (1998). Saccadic localization of random dot targets. Vision Research, 38, 895-910.

McRae, K., Butler, B. E., \& Popiel, S. J. (1987). Spatiotopic and retinotopic components of iconic memory. Psychological Research, 49, 221-227.

Mertens, I., Siegmund, H., \& Grusser, O. (1993). Gaze motor asymmet- 
ries in the perception of faces during a memory task. Neumopsychologia, 31, 989-998.

Meyer, A. S., Sleiderink, A., \& Levelt, W. J. M. (1998). Viewing and naming objects: Eye movements during noun phrase production. $\mathrm{Cog}$ nition, 66, B25-B33.

Miller, L. K. (1978). Development of selective attention during visual search. Developmental Psychology, 14, 439-440.

Miura, T. (1990). Active function of eye movement and useful field of view in a realistic setting. In R. Groner, G. d'Ydewalle, \& R. Parnham (Eds.), From eye to mind: Information acquisition in perception, search, and reading (pp. 119-127). Amsterdam: North Holland.

Moffitt, K. (1980). Evaluation of the fixation duration in visual search. Perception \& Psychophysics, 27, 370-372.

Moray, N., \& Rotenberg, I. (1989). Fault management in process control: Eye movements and action. Ergonomics, 32, 1319-1342.

Morris, R. K. (1994). Lexical and message-level sentence context effects on fixation times in reading. Journal of Experimental Psychology: Learning, Memory, and Cognition, 20, 92-103.

Morris, R. K., \& Folk, J. R. (1998). Focus as a contextual priming mechanism in reading. Memory \& Cognition 26, 1313-1322.

Morris, R. K., \& Rayner, K. (1991). Eye movements in skilled reading: Implications for developmental dyslexia. In J. F. Stein (Ed.), Vision and visual dyslexia (pp. 233-242). London: Macmillan.

Morris, R. K., Rayner, K., \& Pollatsek, A. (1990). Eye movement guidance in reading: The role of parafoveal letter and space information. Journal of Experimental Psychology: Human Perception and Performance, 16, 268-281.

Morrison, R. E. (1983). Retinal image size and the perceptual span in reading. In $\mathrm{K}$. Rayner (Ed.), Eye movements in reading: Perceptual and language processes (pp. 31-40). New York: Academic Press.

Morrison, R. E. (1984). Manipulation of stimulus onset delay in reading: Evidence for parallel programming of saccades. Journal of Experimental Psychology: Human Perception and Performance, 10, $667-682$.

Morrison, R. E., \& Inhoff, A. W. (1981). Visual factors and eye movements in reading. Visible Language, 15, 129-146.

Morrison, R. E., \& Rayner, K. (1981). Saccade size in reading depends upon character spaces and not visual angle. Perception \& Psychophysics, 30, 395-396.

Motter, B. C., \& Belky, E. J. (1998a). The guidance of eye movements during active visual search. Vision Research, 38, 1805-1815.

Motter, B. C., \& Belky, E. J. (1998b). The zone of focal attention during active visual search. Vision Research, 38, 1007-1022.

Mourant, R., \& Rockwell, T. H. (1972). Strategies of visual search by novice and experienced drivers. Human Factors, 14, 325-335.

Muller, P., Cavegn, D., d'Y dewalle, G., \& Groner, R. (1993). A comparison of a new limbus tracker, corneal reflection technique, Purkinje eye tracking and electro-oculography. In G. d'Ydewalle \& J. Van Rensbergen (Eds.), Perception and cognition: Advances in eye movement research (pp. 393-401). Amsterdam: North Holland.

Murray, W. S. (1998). Parafoveal pragmatics. In G. Underwood (Ed.), Eye guidance in reading and scene perception (pp. 181-200). Oxford, England: Elsevier.

Murray, W. S., \& Kennedy, A. (1988). Spatial coding in the processing of anaphor by good and poor readers: Evidence from eye movement analyses. Quarterly Journal of Experimental Psychology, 40A, 693718.

Murray, W. S., \& Liversedge, S. P. (1994). Referential context effects on syntactic processing. In C. Clifton, L. Frazier, \& K. Rayner (Eds.), Perspectives on sentence processing (pp. 359-388). Hillsdale, NJ: Erlbaum.

Murray, W., \& Rowan, M. (1998). Early, mandatory, pragmatic processing. Journal of Psycholinguistic Research, 27, 1-22.

Nattkemper, D., \& Prinz, W. (1984). Costs and benefits of redundancy in visual search. In A. G. Gale \& F. Johnson (Eds.), Theoretical and applied aspects of eye movement research (pp. 343-351). Amsterdam: North Holland.

Nattkemper, D., \& Prinz, W. (1986). Saccade amplitude determines fixation duration: Evidence from continuous search. In J. K. O'Regan \& A. Lévy-Schoen (Eds.), Eye movements: From physiology to cognition (pp. 285-292). Amsterdam: North Holland.

Nazir, T. A. (1991). On the role of refixations in letter strings: The influence of oculomotor factors. Perception \& Psychophysics, 49, 373-389.

Nazir, T. A. (1993). On the relation between the optimal and the preferred viewing position in words during reading. In G. d'Ydewalle \& J. Van Rensbergen (Eds.), Perception and cognition: Advances in eye movement research (pp. 171-180). Amsterdam: North Holland.

Nazir, T. A., Heller, D., \& Sussmann, C. (1992). Letter visibility and word recognition: The optimal viewing position in printed words. Perception \& Psychophysics, 52, 315-328.

Nazir, T. A., \& Jacobs, A. M. (1991). The effects of target discriminability and retinal eccentricity on saccade latencies: An analysis in terms of variable-criterion theory. Psychological Research, 53, 281-289.

Nazir, T. A., O'Regan, J. K., \& Jacobs, A. M. (1991). On words and their letters. Bulletin of the Psychonomic Society, 29, 171-174.

Neary, C., \& Wilkins, A. J. (1989). Effects of phosphor persistence on perception and the control of eye movements. Perception, 18, 257264.

Nelson, W. W., \& Loftus, G. R. (1980). The functional visual field during picture viewing. Journal of Experimental Psychology: Human Learning and Memory, 6, 391-399.

Ni, W., Crain, S., \& Shankweiler, D. (1996). Sidestepping garden paths: Assessing the contributions of syntax, semantics, and plausibility in resolving ambiguities. Language and Cognitive Processes, 11, 283334.

Ni, W., Fodor, J. D., Crain, S., \& Shankweiler, D. (1998). Anomaly detection: Eye movement patterns. Journal of Psycholinguistic Research, 27, 515-540.

Niemann, T., Lappe, M., \& Hoffman, K. (1996). Visual inspection of three-dimensional objects by human observers. Perception, 25, 1027 1042.

Nodine, C. F., Carmody, D. P., \& Herman, E. (1979). Eye movements during visual search for artistically embedded targets. Bulletin of the Psychonomic Society, 13, 371-374.

Nodine, C. F., Carmody, D. P., \& Kundel, H. L. (1978). Searching for Nina. In J. Senders, D. F. Fisher, \& R. Monty (Eds.), Eye movements and the higher psychological functions (pp. 241-258). Hillsdale, NJ: Erlbaum.

Nodine, C. F., Kundel, H. L., Toto, L. C., \& Krupinski, E. A. (1992). Recording and analyzing eye-position data using a microcomputer workstation. Behavior Research Methods, Instruments \& Computers, 24, 475-485.

Noton, D., \& Stark, L. (1971a). Scan paths in eye movements during pattern perception. Science, $171,308-311$.

Noton, D., \& Stark, L. (1971b). Scan paths in saccadic eye movements while viewing and recognizing patterns. Vision Research, 11, 929942.

Noyes, L. (1980). The position of type on maps: The effect of surrounding material on word recognition times. Human Factors, 22, $353-360$.

O'Brien, E. J., Raney, G. E., Albrecht, J. E., \& Rayner, K. (1997). Processes involved in the resolution of explicit anaphors. Discourse Processes, 23, 1-24.

O'Brien, E. J., Shank, D. M., Myers, J. L., \& Rayner, K. (1988). Elaborative inferences during reading: Do they occur on-line? Journal of Experimental Psychology: Learning, Memory, and Cognition, 14, $410-420$. 
Olson, R. K., Conners, F. A., \& Rack, J. P. (1991). Eye movements in dyslexia and normal readers. In J. F. Stein (Ed.), Vision and visual dyslexia (pp. 243-250). London: Macmillan.

Olson, R. K., Kliegl, R., \& Davidson, B. J. (1983a). Dyslexic and normal children's tracking eye movements. Journal of Experimental $P_{s y-}$ chology: Human Perception and Performance, 9, 816-825.

Olson, R. K., Kliegl, R., \& Davidson, B. J. (1983b). Eye movements in reading disability. In $\mathrm{K}$. Rayner (Ed.), Eye movements in reading: Perceptual and language processes (pp. 467-480). New York: Academic Press.

Olson, R. K., Kliegl, R., Davidson, B. J., \& Foltz, G. (1985). Individual differences and developmental differences in reading disability. In G. MacKinnon \& T. G. Waller (Eds.), Reading research: Advances in theory and practice (pp. 1-64). New York: Academic Press.

O'Regan, J. K. (1979). Eye guidance in reading: Evidence for the linguistic control hypothesis. Perception \& Psychophysics, 25, 501-509.

O'Regan, J. K. (1980). The control of saccade size and fixation duration in reading: The limits of linguistic control. Perception \& Psychophysics, 28, 112-117.

O'Regan, J. K. (1981). The convenient viewing position hypothesis. In D. F. Fisher, R. A. Monty, \& J. W. Senders (Eds.), Eye movements: Cognition and visual perception (pp. 289-298). Hillsdale, NJ: Erlbaum.

O'Regan, J. K. (1983). Elementary perceptual and eye movement control processes in reading. In $\mathrm{K}$. Rayner (Ed.), Eye movements in reading: Perceptual and language processes (pp. 121-140). New York: Academic Press.

O'Regan, J. K. (1990). Eye movements and reading. In E. Kowler (Ed.), Eye movements and their role in visual and cognitive processes (pp. 395-453). Amsterdam: Elsevier.

O'Regan, J. K. (1992). Optimal viewing position in words and the strategy-tactics theory of eye movements in reading. In K. Rayner (Ed.), Eye movements and visual cognition: Scene perception and reading (pp. 333-354). New York: Springer-Verlag.

O'Regan, J. K., \& Jacobs, A. M. (1992). The optimal viewing position effect in word recognition: A challenge to current theory. Journal of Experimental Psychology: Human Penception and Performance, 18, 185-197.

O'Regan, J. K., \& Lévy-Schoen, A. (1983). Integrating visual information from successive fixations: Does trans-saccadic fusion exist? $V i$ sion Research, 23, 765-768.

O'Regan, J. K., \& Lévy-Schoen, A. (1987). Eye movement strategy and tactics in word recognition and reading. In M. Coltheart (Ed.), Attention and performance: Vol. 12. The psychology of reading (pp. 363383). Hillsdale, NJ: Erlbaum.

O'Regan, J. K., Lévy-Schoen, A., \& Jacobs, A. M. (1983). The effect of visibility on eye movement parameters in reading. Perception \& Psychophysics, 34, 457-464.

O'Regan, J. K., Lévy-Schoen, A., Pynte, J., \& Brugaillère, B. (1984). Convenient fixation location within isolated words of different length and structure. Joumal of Experimental Psychology: Human Perception and Performance, 10, 250-257.

Osaka, N. (1987). Effect of peripheral visual field size upon eye movements during Japanese text processing. In J. K. O'Regan \& A. LévySchoen (Eds.), Eye movements: From physiology to cognition (pp. 421-429). Amsterdam: North Holland.

Osaka, N. (1989). Eye fixation and saccade during kana and kanji text reading: Comparison of English and Japanese text processing. Bulletin of the Psychonomic Society, 27, 548-550.

Osaka, N. (1992). Size of saccade and fixation duration of eye movements during reading: Psychophysics of Japanese text processing. Journal of the Optical Society of America A, 9, 5-13.

Osaka, N. (1993a). Asymmetry of the effective visual field in vertical reading as measured with a moving window. In G. d'Ydewalle \& J.
Van Rensbergen (Eds.), Perception and cognition: Advances in eye movement research (pp. 275-283). Amsterdam: North Holland.

Osaka, N. (1993b). Reading vertically without a fovea. In S. F. Wright \& R. Groner (Eds.), Facets of dyslexia and its remediation (pp. 257265). Amsterdam: North Holland.

Osaka, N., \& Oda, K. (1991). Effective visual field size necessary for vertical reading during Japanese text processing. Bulletin of the Psychonomic Society, 29, 345-347.

Ottes, F. P., Van Gisbergen, J. A. M., \& Eggermont, J. J. (1984), Metrics of saccade responses to double-stimuli: Two different modes. Vision Research, 24, 1169-1179.

Ottes, F. P., Van Gisbergen, J. A. M., \& Eggermont, J. J. (1985). Latency dependence of color-based target vs nontarget information by the saccadic system. Vision Research, 25, 849-862.

Paap, K. R., \& Newsome, S. L. (1981). Parafoveal information is not sufficient to produce semantic or visual priming. Perception \& Psychophysics, 29, 457-466.

Pacht, J. M., \& Rayner, K. (1993). The processing of homophonic homographs during reading: Evidence from eye movement studies. Journal of Psycholinguistic Research, 22, 252-271.

Palmer, J., \& Ames, C. T. (1992). Measuring the effect of multiple eye fixations on memory for visual attributes. Perception \& Psychophysics, 52, 295-306.

Parker, R. E. (1978). Picture processing during recognition. Journal of Experimental Psychology: Human Perception and Performance, 4. 284-293.

Pashler, H., Carrier, M., \& Hoffman, J. (1993). Saccadic eye movements and dual-task interference. Quarterly Journal of Experimental Psychology, 46A, 51-82.

Patching, G. R., \& Jordan, T. R. (in press). Increasing the benefits of eye-tracking devices in divided visual field studies of cerebral asymmetry. Behavior Research Methods, Instruments, \& Computers.

Paterson, K. B., Liversedge, S. P., \& Underwood, G. (in press). The influence of focus operators on syntactic processing of short relative clause sentences. Quarterly Journal of Experimental Psychology.

Paterson, K. B., Sanford, A. J., Moxey, L. M., \& Dawydiak, E. (1998). Quantifier polarity and referential focus during reading. Journal of Memory and Language, 39, 290-306.

Patla, A. E., \& Vickers, J. N. (1997). Where and when do we look as we approach and step over an obstacle in the travel path? NeuroReport, $8,3661-3665$.

Pavlidis, G. T. (1978). The dyslexics erratic eye movements: Case studies. Dyslexia Review, 1, 22-28.

Pavlidis, G. T. (1981). Do eye movements hold the key to dyslexia? Neuropsychologia, 19, 57-64.

Pavlidis, G. T. (1983). The "dyslexia syndrome'" and its objective diagnosis by erratic eye movements. In $\mathrm{K}$. Rayner (Ed.), Eye movements in reading: Perceptual and language processes (pp. 441-466). New York: Academic Press.

Pavlidis, G. T. (1985). Eye movement differences between dyslexics, normal and slow readers while sequentially fixating digits. American Joumal of Optometry and Physiological Optics, 62, 820-822.

Pavlidis, G. T. (1991) . Diagnostic significance and relationship between dyslexia and erratic eye movements. In J. F. Stein (Ed.), Vision and visual dyslexia (pp. 263-270). London: Macmillan.

Peng, D. L., Orchard, L. N., \& Stern, J. A. (1983). Evaluation of eye movement variables of Chinese and American readers. Pavlovian Journal of Biological Sciences, 18, 94-102.

Perea, M., \& Pollatsek, A. (1998). The effects of neighborhood frequency in reading and lexical decision. Journal of Experimental Psychology: Human Perception and Performance, 24, 767-779.

Perry, A. R., Dember, W. N., Warm, J. S., \& Sacks, J. G. (1989). Letter identification in normal and dyslexic readers: A verification. Bulletin of the Psychonomic Society, 27, 445-448. 
Phillips, M. L., \& David, A. S. (1997). Visual scan paths are abnormal in deluded schizophrenia. Neuropsychologia, 35, 99-105.

Phillips, R. J. (1981). Searching for a target in a random arrangement of names: An eye fixation analysis. Canadian Journal of Psychology, 35, 330-346.

Pickering, M. J., \& Traxler, M. J. (1998). Plausibility and recovery from garden paths: An eye-tracking study. Journal of Experimental Psychology: Learning, Memory, and Cognition, 24, 940-961.

Pillalamarri, R. S., Barnette, B. D., Birkmire, D., \& Karsh, R. (1993). Cluster: A program for the identification of eye-fixation-cluster characteristics. Behavior Research Methods, Instruments, \& Computers, $25,9-15$.

Pirozzolo, F. J., \& Hansch, E. C. (1981). Oculomotor reaction time in dementia reflects degree of cerebral dysfunction. Science, 214, 349351.

Pirozzolo, F. J., \& Rayner, K. (1978). Disorders of oculomotor scanning and graphic orientation in developmental Gerstmann syndrome. Brain and Language, 5, 119-126.

Pirozzolo, F. J., \& Rayner, K. (1979). The neural control of eye movements in acquired and developmental reading disorders. In H. A. Whitaker \& H. A. Whitaker (Eds.), Studies in neurolinguistics (pp. 97123). New York: Academic Press.

Polanka, M. (1995). Factors affecting eye movements during the reading of short melodies. Psychology of Music, 23, 177-183.

Pollatsek, A. (1983). What can eye movements tell us about dyslexia? In K. Rayner (Ed.), Eye movements in reading: Perceptual and language processes (pp. 511-522). New York: Academic Press.

Pollatsek, A. (1993). Eye movements in reading: In D. M. Willows, R.S. Kruk, \& E. Corcos (Eds.), Visual processes in reading and reading disabilities (pp. 191-214). Hillsdale, NJ: Erlbaum.

Pollatsek, A., Bolozky, S., Well, A. D., \& Rayner K. (1981). Asymmetries in the perceptual span for Israeli readers. Brain and Language, 14, 174-180.

Pollatsek, A., Lesch, M., Morris, R. K., \& Rayner, K. (1992). Phonological codes are used in integrating information across saccades in word identification and reading. Journal of Experimental Psychology: $\mathrm{Hu}$ man Perception and Performance, 18, 148-162.

Pollatsek, A., Perea, M., \& Binder, K. S. (in press). The effects of neighborhood size in reading and lexical decision. Journal of Experimental Psychology: Human Perception and Performance.

Pollatsek, A., Raney, G. E., LaGasse, L., \& Rayner, K. (1993). The use of information below fixation in reading and in visual search. Canadian Journal of Experimental Psychology, 47, 179-200.

Pollatsek, A., \& Rayner, K. (1982). Eye movement control in reading: The role of word boundaries. Journal of Experimental Psychology: Human Perception and Performance, 8, 817-833.

Pollatsek, A., \& Rayner, K. (1990). Eye movements and lexical access in reading. In D. A. Balota, G. B. Flores d'Arcais, \& K. Rayner (Eds.), Comprehension processes in reading (pp. 143-164). Hillsdale, NJ: Erlbaum.

Pollatsek, A., \& Rayner, K. (1992). What is integrated across fixations? In K. Rayner (Ed.), Eye movements and visual cognition: Scene perception and reading (pp. 166-191). New York: Springer-Verlag.

Pollatsek, A., Rayner, K., \& Balota, D. A. (1986). Inferences about eye movement control from the perceptual span in reading. Perception \& Psychophysics, 40, 123-130.

Pollatsek, A., Rayner, K., \& Collins, W. E. (1984). Integrating pictorial information across eye movements. Journal of Experimental Psychology: General, 113, 426-442.

Pollatsek, A., Rayner, K., \& Henderson, J. M. (1990). Role of spatial location in integration of pictorial information across saccades. Journal of Experimental Psychology: Human Perception and Performance, 16, 199-210.

Ponsoda, V., Scott, D., \& Findlay, J. M. (1995). Probability vector and transition matrix analysis of eye movements during visual search. Acta Psychologica, 88, 167-185.

Posner, M. I. (1980). Orienting of attention. Quarterly Journal of Experimental Psychology, 32, 3-25.

Previc, F. (1996). Attentional and oculomotor influences on visual field anisotropies in visual search performance. Visual Cognition, 3, 277301.

Prinz, W. (1984). Attention and sensitivity in visual search. Psychological Research, 45, 355-366.

Prinz, W., Nattkemper, D., \& Ullmann, T. (1992). Moment-to-moment control of saccadic eye movements: Evidence from continuous search. In K. Rayner (Ed.), Eye movements and visual cognition: Scene perception and reading (pp. 108-129). New York: Springer-Verlag.

Pynte, J. (1974). Readiness for pronunciation during the reading process. Perception \& Psychophysics, 16, 110-112.

Pynte, J. (1996). Lexical control of within-word eye movements. Journal of Experimental Psychology: Human Perception and Performance, 22, 958-969.

Pynte, J., Kennedy, A., \& Murray, W. S. (1991). Within-word inspection strategies in continuous reading: Time course of perceptual, lexical, and contextual processes. Joumal of Experimental Psychology: $\mathrm{Hu}$ man Perception and Pefformance, 17, 458-470.

Radach, R., \& Kempe, V. (1993). An individual analysis of initial fixation positions in reading. In G. d'Ydewalle \& J. Van Rensbergen (Eds.), Perception and cognition: Advances in eye movement research (pp. 213-226). Amsterdam: North Holland.

Radach, R., Krummenacher, J., Heller, D., \& Hofmesiter, J. (1995). Individual eye movement patterns in word recognition: Perceptual and linguistic factors. In J. M. Findlay, R. Walker, \& R. W. Kentridge (Eds.), Eye movement research: Mechanisms, processes and applications (pp. 421-432). Amsterdam: North Holland.

Radach, R., \& McConkie, G. W. (1998). Determinants of fixation positions in words during reading. In G. Underwood (Ed.), Eye guidance in reading and scene perception (pp. 77-101). Oxford, England: Elsevier.

Rafal, R. D., Calabresi, P. A., Brennan, C. W., \& Sciolto, T. K. (1989). Saccade preparation inhibits reorienting to recently attended locations. Journal of Experimental Psychology: Human Perception and Performance, $15,673-685$.

Rahimi, M., Briggs, R. P., \& Thom, D. R. (1990). A field evaluation of driver eye and head movement strategies toward environmental targets and distractors. Applied Ergonomics, 21, 267-274.

Raney, G. E., \& Rayner, K. (1993). Event-related brain potentials, eye movements, and reading. Psychological Science, 4, 283-286.

Raney, G. E., \& Rayner, K. (1995). Word frequency effects and eye movements during two readings of a text. Canadian Journal of Experimental Psychology, 49, 151-172.

Raymond, J. E., Ogden, N. A., Fagan, J. E., \& Kaplan, B. J. (1988). Fixational instability in dyslexic children. American Joumal of $O p$ tometry and Physiological Optics, 65, 174-181.

Rayner, K. (1975a). Parafoveal identification during a fixation in reading. Acta Psychologica, 39, 272-282.

Rayner, K. (1975b). The perceptual span and peripheral cues in reading. Cognitive Psychology, 7, 65-81.

Rayner, K. (1977). Visual attention in reading: Eye movements reflect cognitive processes. Memory \& Cognition, 4, 443-448.

Rayner, K. (1978a). Eye movement latencies for parafoveally presented words. Bulletin of the Psychonomic Society, 11, 13-16.

Rayner, K. (1978b). Eye movements in reading and information processing. Psychological Bulletin. 85, 618-660.

Rayner, K. (1978c). Foveal and parafoveal cues in reading. In J. Requin (Ed.), Attention and performance (Vol. 7, pp. 149-162). Hillsdale, NJ: Erlbaum. 
Rayner, K. (1979a). Eye guidance in reading: Fixation locations within words. Perception, 8, 21-30.

Rayner, K. (1979b). Eye movements and cognitive psychology: Online computer approaches to studying visual information processing. Behavior Research Methods \& Instruments, 11, 164-171.

Rayner, K. (1983). Eye movements, perceptual span, and reading disability. Annals of Dyslexia, 33, 163-173.

Rayner, K. (1984). Visual selection in reading, picture perception, and visual search: A tutorial review. In H. Bouma \& D. Bouwhuis (Eds.), Attention and performance (Vol. 10). Hillsdale, NJ: Erlbaum.

Rayner, K. (1985a). Do faulty eye movements cause dyslexia? Developmental Neuropsychology, 1, 3-15.

Rayner, K. (1985b). The role of eye movements in learning to read and reading disability. Remedial and Special Education, 6, 53-60.

Rayner, K. (1986). Eye movements and the perceptual span in beginning and skilled readers. Journal of Experimental Child Psychology, 41, 211-236.

Rayner, K. (1993). Eye movements in reading: Recent developments. Current Directions in Psychological Science, 2, 81-85.

Rayner, K. (1995). Eye movements and cognitive processes in reading, visual search, and scene perception. In J. M. Findlay, R. Walker; \& R. W. Kentridge (Eds.), Eye movement research: Mechanisms, processes and applications (pp. 3-22). Amsterdam: North Holland.

Rayner, K. (1997). Understanding eye movements in reading. Scientific Studies of Reading, 1, 301-323.

Rayner, K., Balota, D. A., \& Pollatsek, A. (1986). Against parafoveal semantic preprocessing during eye fixations in reading. Canadian Journal of Psychology, 40, 473-483.

Rayner, K., \& Bertera, J. H. (1979). Reading without a fovea. Science, 206, 468-469.

Rayner, K., Carlson, A., \& Frazier, L. (1983). The interaction of syntax and semantics during sentence processing: Eye movements in the analysis of semantically biased sentences. Journal of Verbal Learning and Verbal Behavior, 22, 358-374.

Rayner, K., \& Duffy, S. A. (1986). Lexical complexity and fixation times in reading: Effects of word frequency, verb complexity, and lexical ambiguity. Memory \& Cognition, 14, 191-201.

Rayner, K., \& Duffy, S. A. (1988). On-line comprehension processes and eye movements in reading. In M. Daneman, G. E. MacKinnon, \& T. G. Waller (Eds.), Reading research: Advances in theory and practice (pp. 13-66). New York: Academic Press.

Rayner, K., \& Fischer, M. H. (1996). Mindless reading revisited: Eye movements during reading and scanning are different. Perception \& Psychophysics, 58, 734-747.

Rayner, K., Fischer, M. H., \& Pollatsek, A. (1998). Unspaced text interferes with both word identification and eye movement control. Vision Research, 38, 1129-1144.

Rayner, K., \& Fisher, D. L. (1987a). Eye movements and the perceptual span during visual search. In J. K. O'Regan \& A. Lévy-Schoen (Eds.), Eye movements: From physiology to cognition (pp. 293-302). Amsterdam: North Holland.

Rayner, K., \& Fisher, D. L. (1987b). Letter processing during eye fixations in visual search. Perception \& Psychophysics, 42, 87-100.

Rayner, K., \& Frazier, L. (1987). Parsing temporarily ambiguous complements. Quarterly Journal of Experimental Psychology, 39A, 657673.

Rayner, K., \& Frazier, L. (1989). Selection mechanisms in reading lexically ambiguous words. Journal of Experimental Psychology: Learning, Memory, and Cognition, 15, 779-790.

Rayner, K., Garrod, S. C., \& Perfetti, C. A. (1992). Discourse influences during parsing are delayed. Cognition, 45, 109-139.

Rayner, K., Inhoff, A. W., Morrison, R., Slowiaczek, M. L., \& Bertera, J. H. (1981). Masking of foveal and parafoveal vision during eye fixations in reading. Journal of Experimental Psychology: Human Perception and Performance, 7, 167-179.

Rayner, K., \& McConkie, G. W. (1976). What guides a reader's eye movements. Vision Research, 16, 829-837.

Rayner, K., McConkie, G. W., \& Ehrlich, S. F. (1978). Eye movements and integrating information across fixations. Journal of Experimental Psychology: Human Perception and Performance, 4, 529-544.

Rayner, K., McConkie, G. W., \& Zola, D. (1980). Integrating information across eye movements. Cognitive Psychology, 12, 206-226.

Rayner, K., \& Morris, R. K. (1990). Do eye movements reflect higher order processes in reading?'In R. Groner, G. d'Ydewalle, \& R. Parnham (Eds.), From eye to mind: Information acquisition in perception, search, and reading (pp. 170-190). Amsterdam: North Holland.

Rayner, K., \& Morris, R. K. (1992). Eye movement control in reading: Evidence against semantic preprocessing. Joumal of Experimental Psychology: Human Perception and Performance, 18, 163-172.

Rayner, K., \& Morrison, R. M. (1981). Eye movements and identifying words in parafoveal vision. Bulletin of the Psychonomic Society, 17, 135-138.

Rayner, K., Murphy, L. A., Henderson, J. M., \& Pollatsek, A. (1989). Selective attentional dyslexia. Cognitive Neuropsychology, 6, $357-$ 378.

Rayner, K., Pacht, J. M., \& Duffy, S. A. (1994). Effects of prior encounter and global discourse bias on the processing of lexically ambiguous words: Evidence from eye fixations. Journal of Memory and Language, 33, 527-544.

Rayner, K., \& Pollatsek, A. (1981). Eye movement control during reading: Evidence for direct control. Quarterly Journal of Experimental Psychology, 33A, 351-373.

Rayner, K., \& Pollatsek, A. (1983). Is visual information integrated across saccades? Perception \& Psychophysics, 34, 39-48.

Rayner, K., \& Pollatsek, A. (1987). Eye movements in reading: A tutorial review. In M. Coltheart (Ed.), Attention and performance (Vol. 12, pp. 327-362). London: Erlbaum.

Rayner, K., \& Pollatsek, A. (1989). The psychology of reading. Englewood Cliffs, NJ: Prentice Hall.

Rayner, K., \& Pollatsek, A. (1992). Eye movements and scene perception. Canadian Iournal of Psychology, 46, 342-376.

Rayner, K., \& Pollatsek, A. (1996). Reading unspaced text is not easy: Comments on the implications of Epelboim et al.'s study for models of eye movement control in reading. Vision Research, 36, 461-470.

Rayner, K., \& Pollatsek, A. (1997). Eye movements, the eye-hand span, and the perceptual span during sight-reading of music. Current Directions in Psychological Science, 6, 49-53.

Rayner, K., Pollatsek, A., \& Bilsky, A. B. (1995). Can a temporal processing deficit account for dyslexia? Psychonomic Bulletin \& Review, 2, 501-507.

Rayner, K., Pollatsek, A., \& Binder, K. S. (1998). Phonological codes and eye movements in reading. Journal of Experimental Psychology: Learning, Memory, and Cognition, 24, 476-497.

Rayner, K., \& Raney, G. E. (1996). Eye movement control in reading and visual search: Effects of word frequency. Psychonomic Bulletin \& Review, 3, 238-244.

Rayner, K., Raney, G. E., \& Pollatsek, A. (1995). Eye movements and discourse processing. In R. F. Lorch \& E. J. O'Brien (Eds.), Sources of coherence in reading (pp. 9-36). Hillsdale, NJ: Erlbaum.

Rayner, K., Reichle, E. D., \& Pollatsek, A. (1998). Eye movement control in reading: An overview and model. In G. Underwood (Ed.), Eye guidance in reading and scene perception (pp. 243-268). Oxford, England: Elsevier.

Rayner, K., \& Sereno, S. C. (1994a). Eye movements in reading: Psycholinguistic studies. In M. Gernsbacher (Ed.), Handbook of psycholinguistics (pp. 57-82). New York: Academic Press. 
Rayner, K., \& Sereno, S. C. (1994b). Regression-contingent analyses: A reply to Altmann. Memory \& Cognition, 22, 291-292.

Rayner, K., \& Sereno, S. C. (1994c). Regressive eye movements and sentence parsing: On the use of regression-contingent analyses. Memory \& Cognition, 22, 281-285.

Rayner, K., Sereno, S. C., Lesch, M. F., \& Pollatsek, A. (1995). Phonological codes are automatically activated during reading: Evidence from an eye movement priming paradigm. Psychological Science, 6, 26-32.

Rayner, K., Sereno, S. C., Morris, R. K., Schmauder, A. R., \& Clifton, C. (1989). Eye movements and on-line language comprehension processes [Special issue]. Language and Cognition Processes, 4, $21-$ 49.

Rayner, K., Sereno, S. C., \& Raney, G. E. (1996). Eye movement control in reading: A comparison of two types of models. Journal of Experimental Psychology: Human Perception and Performance, 22, 11881200.

Rayner, K., Slowiaczek, M. L., Clifton, C., \& Bertera, J. H. (1983). Latency of sequential eye movements: Implications for reading. Journal of Experimental Psychology: Human Perception and Performance, 9, 912-922.

Rayner, K., \& Well, A. D. (1996). Effects of contextual constraint on eye movements in reading: A further examination. Psychonomic Bulletin \& Review, 3, 504-509.

Rayner, K., Well, A. D., \& Pollatsek, A. (1980). Asymmetry of the effective visual field in reading. Perception \& Psychophysics, 27, 537-544.

Rayner, K., Well, A. D., Pollatsek, A., \& Bertera, J. H. (1982). The availability of useful information to the right of fixation in reading. Perception \& Psychophysics, 31, 537-550.

Reder, S. M. (1973). On-line monitoring of eye position signals in contingent and noncontingent paradigms. Behavior Research Methods and Instrumentation, 5, 218-228.

Reichle, E. D., Pollatsek, A., Fisher, D. L., \& Rayner, K. (1998). Toward a model of eye movement control in reading. Psychological Review, 105, 125-157.

Reilly, R. (1993). A connectionist framework for modeling eye-movement control in reading. In G. d'Ydewalle \& J. Van Rensbergen (Eds.), Perception and cognition: Advances in eye movement research (pp. 191-212). Amsterdam: North Holland.

Reilly, R., \& O'Regan, J. K. (1998). Eye movement control in reading: A simulation of some word-targeting strategies. Vision Research, 38, 303-317.

Remington, R. W. (1980). Attention and saccadic eye movements. Journal of Experimental Psychology: Human Perception and Performance, 6, 726-744.

Reuter-Lorenz, P. A., \& Fendrich, R. (1992). Oculomotor readiness and covert orienting: Differences between central and peripheral cues. Perception \& Psychophysics, 52, 336-344.

Riggs, L. A., Merton, P. A., \& Morton, H. B. (1974). Suppression of visual phosphenene during saccadic eye movements. Vision Research, 14, 997-1010.

Ritter, M. (1976). Evidence for visual persistence during saccadic eye movements. Psychological Research, 39, 67-85.

Rizzo, M., \& Hurtig, R. (1992). Visual search in hemineglect: What stirs idle eyes? Clinical Visual Science, 7, 39-52.

Roberts, R. J., Hager, L. D., \& Heron, C. (1994). Prefrontal cognitive processes: Working memory and inhibition in the antisaccade task. Journal of Experimental Psychology: General, 123, 374-393.

Ross, L. E., \& Ross, S. M. (1980). Saccade latency and warning signals: Stimulus onset, offset and change as warning events. Perception \& Psychophysics, 27, 251-257.

Ross, S. M., \& Ross, L. E. (1981). Saccade latency and warning signals:
Effects of auditory and visual offset and onset. Perception \& Psychophysics, 29, 429-437.

Rothkopf, E. Z. (1978). Analyzing eye movements to infer processing styles during learning from text. In J. W. Senders, D. F. Fisher, \& R. A. Monty (Eds.), Eye movements and the higher psychological functions (pp. 209-224). Hillsdale, NJ: Erlbaum.

Rothkopf, E. Z., \& Billington, M. J. (1979). Goal-guided learning from text: Inferring a descriptive processing model from inspection times and eye movements. Journal of Educational Psychology, 3, 310-327.

Rubin, G. S., \& Turano, K. (1992). Reading without saccadic eye movements. Vision Research, 32, 895-902.

Ruggieri, V., \& Fernandez, M. F. (1994). Gaze orientation in perception of reversible figures. Perceptual and Motor Skills, 78, 299-303.

Saida, S., \& Ikeda, M. (1979). Useful field size for pattern perception. Perception \& Psychophysics, 25, 119-125.

Salthouse, T. A. (1984). Effects of age and skill in typing. Journal of Experimental Psychology: General, 113, 345-371.

Salthouse, T. A., \& Ellis, C. L. (1980). Determinants of eye-fixation duration. American Journal of Psychology, 93, 207-234.

Salthouse, T. A., Ellis, C. L., Diener, D. C., \& Somberg, B. L. (1981). Stimulus processing during eye fixations. Joumal of Experimental Psychology: Human Perception and Performance, 7, 611-623.

Sanders, A. F. (1993). Processing information in the functional visual field. In G. d'Ydewalle \& J. Van Rensbergen (Eds.), Perception and cognition: Advances in eye movement research (pp. 3-22). Amsterdam: North Holland.

Sanders, A. F., \& Houtmans, M. J. M. (1985). There is no central stimulus encoding during saccadic eye shifts: A case against general parallel processing notions. Acta Psychologica, 60, 323-338.

Sanders, A. F., \& Rath, A. M. (1991). Perceptual processing and speedaccuracy trade-off. Acta Psychologica, 77, 275-291.

Sanders, A. F., \& van Duren, L. L. (1998). Stimulus control of visual fixation duration in a single saccade paradigm. Acta Psychologica, 99, $163-176$

Schilling, H. E. H., Rayner, K., \& Chumbley, J. I. (1998). Comparing naming, lexical decision, and eye fixation times: Word frequency effects and individual differences. Memory \& Cognition, 26, 12701281.

Schmauder, A. R. (1991). Argument structure frames: A lexical complexity measure. Journal of Experimental Psychology: Learning, Memory, and Cognition, 17, 49-65.

Schmauder, A. R. (1992). Eye movements and reading processes. In K. Rayner (Ed.), Eye movements and visual cognition: Scene perception and reading (pp. 369-378). New York: Springer-Verlag.

Schmauder, A. R., \& Egan, M. C. (1998). The influence of semantic fit on on-line sentence processing. Memory \& Cognition, 26, 1304-1312.

Schroyens, W., Vitu, F., Brysbaert, M., \& d'Ydewalle, G. (in press). Visual attention and eye movement control during reading: The case of parafoveal processing. Quarterly Journal of Experimental Psychology:

Schustack, M. W., Ehrlich, S. F., \& Rayner, K. (1987). The complexity of contextual facilitation in reading: Local and global influences. Journal of Memory and Language, 26, 322-340.

Scialfa, C. T., \& Joffe, K. M. (1998). Response times and eye movements in feature search and conjunction search as a function of target eccentricity. Perception \& Psychophysics, 60, 1067-1082.

Scialfa, C. T., Thomas, D. M., \& Joffe, K. M. (1994). Age differences in the useful field of view: An eye movement analysis. Optometry and Vision Science, 7I, 1-7.

Scinto, L. F. (1978). Relation of eye fixations to old-new information in texts. In J. W. Senders, D. F. Fisher, \& R. A. Monty (Eds.), Eye movements and the higher psychological functions (pp. 175-194). Hillsdale, NJ: Erlbaum.

Scinto, L. F., \& Barnette, B. D. (1986). An algorithm for determining 
clusters, pairs or singletons in eye-movement scan-path records. Behavior Research Methods, Instruments, \& Computers, 18, 41-44.

Scinto, L. F., Pillalamarri, R., \& Karsh, R. (1986). Cognitive strategies for visual search. Acta Psychologica, 62, 263-294.

Sclingensiepen, K. H., Campbell, F. W., Legge, G. E., \& Walker, T. D. (1986). The importance of eye movements in the analysis of simple patterns. Vision Research, 26, 1111-1117.

Scotto, M. A., Oliva, G. A., \& Tuccio, M. T. (1990). Eye movements and reversal rates of ambiguous figures. Perceptual and Motor Skills, $70,1059-1073$.

Sereno, A. B. (1992). Programming saccades: The role of attention. In $\mathrm{K}$. Rayner (Ed.), Eye movements and visual cognition: Scene perception and reading (pp. 89-107). New York: Springer-Verlag.

Sereno, A. B., \& Holzman, P. S. (1993). Express saccades and smooth pursuit eye movement functions in schizophrenia, affective disorders, and normal subjects. Journal of Cognitive Neuroscience, 5, 303-316.

Sereno, S. C. (1992). Early lexical effects when fixating a word in reading. In $\mathrm{K}$. Rayner (Ed.), Eye movements and visual cognition: Scene perception and reading (pp. 304-316). New York: SpringerVerlag.

Sereno, S. C. (1995). Resolution of lexical ambiguity: Evidence from an eye movement priming paradigm. Journal of Experimental Psychology: Learning, Memory, and Cognition, 21, 582-595.

Sereno, S. C., Pacht, J. M., \& Rayner, K. (1992). The effect of meaning frequency on processing lexically ambiguous words: Evidence from eye fixations. Psychological Science, 3, 296-300.

Sereno, S. C., \& Rayner, K. (1992). Fast priming during eye fixations in reading. Journal of Experimental Psychology: Human Perception and Performance, 18, 173-184.

Sereno, S. C., Rayner, K., \& Posner, M. I. (1998). Establishing a timeline of processing during reading: Evidence from eye movements and event-related potentials. NeuroReport, 9, 2195-2200.

Shaffer, L. H. (1973). Latency mechanisms in transcription. In S. Kornblum (Ed.), Attention and performance (Vol. 4, pp. 435-446). New York: Academic Press.

Shank, M. D., \& Haywood, K. M. (1987). Eye movements while viewing a baseball pitch. Perceptual and Motor Skills, 64, 1191-1197.

Shapiro, K. L., \& Raymond, J. E. (1989). Training efficient oculomotor strategies enhances skill acquisition. Acta Psychologica, 71, 217242.

Shebilske, W. L., \& Fisher, D. F. (1983). Eye movements and context effects during reading of extended discourse. In K. Rayner (Ed.), Eye movements in reading: Perceptual and language processes (pp. 153179). New York: Academic Press.

Shepherd, M., Findlay, J. M., \& Hockey, R. J. (1986). The relationship between eye movements and spatial attention. Quarterly Journal of Experimental Psychology, 38A, 475-491.

Shinar, D., McDowell, E. D., \& Rockwell, T. H. (1977). Eye movements in curve negotiation. Human Factors, 19, 63-71.

Shiori, S. (1993). Postsaccadic processing of the retinal image during picture scanning. Perception \& Psychophysics, 53, 305-314.

Shioiri, S., \& Ikeda, M. (1989). Useful resolution for picture perception as a function of eccentricity. Perception, 18, 347-361.

Slaghuis, W. L., Lovegrove, W. J., \& Freestun, J. (1992). Letter recognition in peripheral vision and metacontrast masking in dyslexic and normal readers. Clinical Vision Sciences, 7, 53-65.

Slowiaczek, M. L. (1983). What does the mind do while the eyes are gazing? In K. Rayner (Ed.), Eye movements in reading: Perceptual and language processes (pp. 345-358). New York: Academic Press.

Slowiaczek, M. L., \& Rayner, K. (1987). Sequential masking during eye fixations in reading. Bulletin of the Psychanomic Society, 25, $175-178$.

Solan, H. A. (1985). Deficient eye movement patterns in achieving high school students: Three case histories. Journal of Learning Disabilities, 18, 66-70.

Solan, H. A., Feldman, J., \& Tujak, L. (1995). Developing visual and reading efficiency in older adults. Optometry and Vision Science, 72, $139-145$.

Speer, S., \& Clifton, C. (1998). Plausibility and argument structure in sentence comprehension. Memory \& Cognition, 26, 965-978.

Spragins, A. B., Lefton, L. A., \& Fisher, D. F. (1976). Eye movements while reading spatially transformed text: A developmental study. Memory \& Cognition, 4, 36-42.

Staller, J., \& Sekuler, R. (1980). Eye movements and choice times in mirror image pattern discrimination. American Journal of Psychology, $93,665-681$.

Stampe, D. M., \& Reingold, E. M. (1995). Selection by looking: A novel computer interface and its application to psychological research. In J. M. Findlay, R. Walker, \& R. W. Kentridge (Eds.), Eye movement research: Mechanisms, processes and applications (pp. 467-478). Amsterdam: North Holland.

Stanley, G. (1994). Eye movements in dyslexic and normal children. In J. Ygge \& G. Lennerstrand (Eds.), Eye movements in reading (pp. 261-271). Oxford, England: Pergamon Press.

Stanley, G., Smith, G. A., \& Howell, E. A. (1983a). Eye movements and sequential tracking in dyslexic and control children. British Journal of Psychology, 74, 181-187.

Stanley, G., Smith, G. A., \& Howell, E. A. (1983b). Eye movements in dyslexic children: Comments on Pavlidis' reply. British Journal of Psychology, 74, 195-197.

Stark, L., \& Ellis, S. R. (1981). Scanpaths revisited: Cognitive models direct active looking. In D. F. Fisher, R. A. Monty, \& J. W. Senders (Eds.), Eye movements: Cognition and visual perception (pp. 193226). Hillsdale, NJ: Erlbąum.

Stein, J. F. (1989). Unstable vergence control and specific reading impairment. British Journal of Ophthalmology, 73, 49.

Stein, J. F. (1994). Binocular control in dyslexics. In J. Ygge \& G. Lennerstrand (Eds.), Eye movements in reading (pp. 273-290). Oxford, England: Pergamon Press.

Stein, J. F., Riddell, P., \& Fowler, M. S. (1988). Disordered vergence eye movement control in dyslexic children. British Journal of Ophthalmology, 72, 162-166.

Stelmach, L. B., Campsall, J. M., \& Herdman, C. M. (1997). Attention and ocular movements. Journal of Experimental Psychology: Human Perception and Performance, 23, 823-844.

Sun, F., Morita, M., \& Stark, L. W. (1985). Comparative patterns of reading eye movement in Chinese and English. Perception \& Psychophysics, 37, 502-506.

Suppes, P. (1990). Eye-movement models for arithmetic and reading performance. In E. Kowler (Ed.), Eye movements and their role in visual and cognitive processes (pp. 455-477). Amsterdam: Elsevier.

Suppes, P. (1994). Stochastic models of reading. In J. Ygge \& G. Lennerstrand (Eds.), Eye movements in reading (pp. 349-364). Oxford, England: Pergamon Press.

Suppes, P., Cohen, M., Laddaga, R., Anliker, J., \& Floyd, R. (1983). A procedural theory of eye movements in doing arithmetic. Journal of Mathematical Psychology, 27, 341-369.

Tanenhaus, M. K., \& Spivey-Knowlton, M. J. (1996). Eye-tracking. Language and Cognitive Processes, 11, 583-588.

Tanenhaus, M. K., Spivey-Knowlton, M. J., Eberhard, K. M., \& Sedivy, J. C. (1995). Integration of visual and linguistic information during spoken language comprehension. Science, 268, 1632-1634.

Tanenhaus, M. K., Spivey-Knowlton, M. J., Eberhard, K. M., \& Sedivy, J. C. (1996). Using eye movements to study spoken language comprehension. In T. Inui \& J. McClelland (Eds.), Attention and performance (Vol. 16, pp. 457-478). Cambridge, MA: MIT Press. 
Taylor, E. A. (1966). The fundamental reading skill. Springfield, IL: Charles C Thomas.

Taylor, S. E. (1965). Eye movements while reading: Facts and fallacies. American Educational Research Journal, 2, 187-202.

Theeuswes, J., Kramer, A. F., Hahn, S., \& Irwin, D. E. (1998). Our eyes do not always go where we want them to go: Capture of the eyes by new objects. Psychological Science, 9, 379-385.

Thibadean, R., Just, M. A., \& Carpenter, P. A. (1982). A model of the time course and content of human reading. Cognitive Science, 6, 101-155.

Tinker, M. A. (1939). Reliability and validity of eye-movement measures of reading. Journal of Experimental Psychology, 19, 732-746.

Tinker, M. A. (1946). The study of eye movements in reading. Psychological Bulletin, 43, 93-120.

Tinker, M. A. (1958). Recent studies of eye movements in reading. Psychological Bulletin, 55, 215-231.

Togami, H. (1984). Affects of visual search performance on individual differences in fixation time and number of fixations. Engonomics, 27, 789-799.

Traxlex, M. J., Bybee, M., \& Pickering, M. J. (1997). Influence of connectives on language comprehension: Eye-tracking evidence for incremental interpretation. Quarterly Joumal of Experimental Psychology, 50A, 481-497.

Traxler, M. J., \& Pickering, M. J. (1996a). Case marking in the parsing of complement sentences: Evidence from eye movements. Quarterly Journal of Experimental Psychology, 49A, 991-1004.

Traxler, M. J., \& Pickering, M. J. (1996b). Plausibility and the processing of unbounded dependencies: An eye-tracking study. Journal of Memory and Language, 35, 454-475.

Trueswell, J. C., Tanenhaus, M. K., \& Garnsey, S. M. (1994). Semantic influences on parsing: Use of thematic role information in syntactic ambiguity resolution. Journal of Memory and Language, 33, 285-318.

Trueswell, J. C., Tanenhaus, M. K., \& Kello, C. (1993). Verb-specific constraints in sentence processing: Separating effects of lexical preference from garden-paths. Journal of Experimental Psychology: Learning, Memory, and Cognition, 19, 528-553.

Truitt, F. E., Clifton, C., Pollatsek, A., \& Rayner, K. (1997). The perceptual span and the eye-hand span in sight reading music. Visual Cognition, 4, 143-162.

Tversky, B. ( 1974). Eye fixations in prediction of recognition and recall. Memory \& Cognition, 2, 275-278.

Underwood, G. (1985). Eye movements during the comprehension of written language. In A. W. Ellis (Vol. Ed.), Progress in the psychology of language (Vol. 2, pp. 45-71). London: Erlbaum.

Underwood, G., Bloomfield, R., \& Clews, S. (1988). Information influences the pattern of eye fixations during sentence comprehension. Perception, 17, 267-278.

Underwood, G., Clews, S., \& Everatt, J. (1990). How do readers know where to look next? Local information distributions influence eye fixations. Quarterly Joumal of Experimental Psychology, 42A, 39-65.

Underwood, G., Clews, S., \& Wilkinson, H. (1989). Eye fixations are influenced by the distribution of information within words. Acta Psychologica, 72, 263-280.

Underwood, G., Hubbard, A., \& Wilkinson, H. (1990). Eye fixations predict reading comprehension: The relationship between reading skilt, reading speed and visual inspection. Language and Speech, 33, 69-81.

Underwood, G., Hyönä, J., \& Niemi, P. (1987). Scanning patterns on individual words during the comprehension of sentences. In J. K. O'Regan \& A. Lévy-Schoen (Eds.), Eye movements: From physiology to cognition (pp. 467-477). Amsterdam: North Holland.

Underwood, N. R., \& McConkie, G. W. (1985). Perceptual span for letter distinctions during reading. Reading Research Quarterly, 20, 153-162.

Underwood, N. R., \& Zola, D. (1986). The span of letter recognition of good and poor readers. Reading Research Quarterly, 21, 6-19.

Uttal, W. R., \& Smith, P. (1968). Recognition of alphabetic characters during voluntary eye movements. Perception \& Psychophysics, 3, 257-264.
Van der Heijden, A. H. C., Bridgeman, B., \& Mewhort, D. J. K. (1986). Is stimulus persistence affected by eye movements? A critique of Davidson, Fox, and Dick (1973). Psychological Research, 48, 179-181.

van Diepen, P. M. J. (1997). A pixel-resolution video switcher for eye contingent display changes. Spatial Vision, 10, 335-344.

van Diepen, P. M. J. (1998a). Foveal stimulus degradation in scene perception. Manuscript submitted for publication.

van Diepen, P. M. J. (1998b). Peripheral versus foveal information processing in scene perception. Manuscript submitted for publication.

van Diepen, P. M. J., DeGraef, P., \& d'Ydewalle, G. (1995). Chronometry of foveal information extraction during scene perception. In J. M. Findlay, R. Walker, \& R. W. Kentridge (Eds.), Eye movement research: Mechanisms, processes and applications (pp. 349-362). Amsterdam: North Holland.

van Diepen, P. M. J., DeGraef, P., \& Van Rensbergen, J. (1994). Online control of moving masks and windows on a complex background using the ATVista Videographics Adapter. Behavior Research Methods, Instruments, and Computers, 24, 454-460.

van Diepen, P. M. J., Wampers, M., \& d'Ydewalle, G. (1998). Functional division of the visual field: Moving masks and moving windows. In G. Underwood (Ed.), Eye guidance in reading and scene perception (pp. 337-356). Oxford, England: Elsevier.

Van Duren, L. (1993). Central stimulus processing during saccadic eye movements. In G. d'Ydewalle \& J. Van Rensbergen (Eds.), Perception and cognition: Advances in eye movement research (pp. 23-36). Amsterdam: North Holland.

Van Duren, L., \& Sanders, A. F. (1992). The output code of a visual fixation. Bulletin of the Psychonomic Society, 30, 305-308.

Van Duren, L., \& Sanders, A. F. (1995). Signal processing during and across saccades. Acta Psychologica, 89, 121-147.

Van Oeffelen, M. P., \& Vos, P. G. (1984a). Enumeration of dots: An eye movement analysis. Memory \& Cognition, 12, 607-612.

Van Oeffelen, M. P., \& Vos, P. G. (1984b). The young child's processing of dot patterns: A chronometric and eye movement analysis. International Journal of Behavioral Development, 7, 53-66.

Vatikiotis-Bateson, E., Eigsti, I., Yano, S., \& Munhall, K. G. (1998). Eye movement of perceivers during audiovisual speech perception. Perception \& Psychophysics, 60, 926-940.

Vaughan, J. (1982). Control of fixation duration in visual search and memory search: Another look. Journal of Experimental Psychology: Human Perception and Performance, 8, 709-723.

Vaughan, J. (1983). Saccadic reaction time in visual search. In K. Rayner (Ed.), Eye movements in reading: Perceptual and language processes (pp. 397-411). New York: Academic Press.

Vaughan, J., \& Graefe, T. M. (1977). Delay of stimulus presentation after the saccade in visual search. Perception \& Psychophysics, 22, 201-205.

Vauras, M., Hyönä, J., \& Niemi, P. (1992). Comprehending coherent and incoherent texts: Evidence from eye movement patterns and recall performance. Journal of Research in Reading, 15, 39-54.

Verfaillie, K. (1997). Transsaccadic memory for the egocentric and allocentric position of a biological-motion walker. Journal of Experimental Psychology: Learning. Memory, and Cognition, 23, 739-760.

Verfaillie, K., De Troy, A., \& Van Rensbergen, J. (1994). Transsaccadic integration of biological motion. Joumal of Experimental Psychology: Learning, Memory, and Cognition, 20, 649-670.

Verschaffel, L., De Corte, E., \& Pauwels, A. (1992). Solving compare problems: An eye movement test of Lewis and Mayer's consistency hypothesis. Journal of Educational Psychology, 84, 85-94.

Vickers, J. N. (1988). Knowledge structures of expert-novice gymnasts. Human Movement Science, 7, 47-72.

Vickers, J. N. (1992). Gaze control in putting. Perception, 21, 117132.

Vickers, J. N. ( 1995). Gaze control in basketball foul shooting. In J. M. Findlay, R. Walker, \& R. W. Kentridge (Eds.), Eye movement re- 
search: Mechanisms, processes and applications (pp. 527-541). Amsterdam: North Holland.

Vickers, J. N. (1996). Visual control when aiming at a far target. Journal of Experimental Psychology: Human Perception and Performance, 22, 342-354.

Vitu, F. (1991a). Against the existence of a range effect during reading. Vision Research, 31, 2009-2015.

Vitu, F. (1991b). The existence of a center of gravity effect in reading. Vision Research, 31, 1289-1313.

Vitu, F. (1991c). The influence of parafoveal processing and linguistic context on the optimal landing position effect. Perception \& Psycho physics, 50, 58-75.

Vitu, F. (1993). The influence of the reading rhythm on the optimal landing position effect. In G. d'Ydewalle \& J. Van Rensbergen (Eds.), Perception and cognition: Advances in eye movement research (pp. 181-192). Amsterdam: North Holland.

Vitu, F., McConkie, G. W., \& Zola, D. (1998). About regressive saccades in reading and their relation to word identification. In G. Underwood (Ed.), Eye guidance in reading and scene perception (pp. 101-124). Oxford, England: Elsevier.

Vitu, F., \& O'Regan, J. K. (1988). Effects of parafoveal processing and reading rhythm on optimal landing position in words of different length and frequency. In G. Luer, U. Lass, \& J. Shallo-Hoffmann (Eds.), Eye movement research: Physiological and psychological aspects (pp. 286-292). Toronto: Hofgrefe.

Vitu, F., \& O'Regan, J. K. (1991). Is there an optimal landing position in words during reading of texts? In R. Schmid \& D. Zambarbieri (Eds.), Oculomotor control and cognitive processes: Normal and pathological aspects (pp. 341-352). Amsterdam: North Holland.

Vitu, F., \& O'Regan, J. K. (1995). A challenge to current theories of eye movements in reading. In J. M. Findlay, R. Walker, \& R. W. Kentridge (Eds.), Eye movement research: Mechanisms, processes, and applications (pp. 381-393). Amsterdam: North Holland.

Vitu, F., O'Regan, J. K., Inhoff, A. W., \& Topolski, R. (1995). Mindless reading: Eye movement characteristics are similar in scanning letter strings and reading text. Perception \& Psychophysics, 57, 352-364.

Vitu, F., O'Regan, J, K., \& Mittau, M. (1990). Optimal landing position in reading isolated words and continuous text. Perception \& Psychophysics, 47, 583-600.

Viviani, P. (1990). Eye movements in visual search: Cognitive, perceptual, and motor control aspects. In E. Kowler (Ed.), Eye movements and their role in visual and cognitive processes (pp. 353-393). Amsterdam: Elsevier.

Viviani, P., \& Swensson, R. G. (1982). Saccadic eye movements to peripherally discriminated visual targets. Journal of Experimental Psychology: Human Perception and Performance, 8, 113-126.

Vonk, W. (1984). Eye movements during comprehension of pronouns. In A. G. Gale \& F. Johnson (Eds.), Theoretical and applied aspects of eye movement research (pp. 203-212). Amsterdam: North Holland.

Vonk, V. (1985). On the purpose of reading and the immediacy of processing pronouns. In R. Groner, G. W. McConkie, \& C. Menz (Eds.), Eye movements and human information processing (pp. 207218). Amsterdam: North Holland.

Walker, R., \& Findlay, J. M. (1996). Saccadic eye movement programming in unilateral neglect. Neuropsychologia, 34, 493-508.

Walker, R., Findlay, J. M., Young, A. W., \& Lincoln, N. B. (1996). Saccadic eye movements in object-based neglect. Cognitive Neuropsychology, 13, 569-615.

Walker, R., \& Young, A. W. (1996). Object-based neglect: An investigation of the contributions of eye movements and perceptual completion. Cortex, 32, 279-295.

Walker-Smith, G. J., Gale, A. G., \& Findlay, J. M. (1977). Eye movement strategies in face perception. Perception, 6, 313-326.
Wampers, M., van Diepen, P. M. J., \& d'Ydewalle, G. (1998). The use of coarse and fine peripheral information during scene perception. Manuscript submitted for publication.

Waters, A. J., Underwood, G., \& Findlay, J. M. (1997). Studying expertise in music reading: Use of a pattern-matching paradigm. Perception \& Psychophysics, 59, 477-488.

Weaver, H. E. (1943). A survey of visual processes in reading differently constructed musical selections. Psychological Monographs, 55, 1-30.

Well, A. D. (1983). Perceptual factors in reading. In K. Rayner (Ed.), Eye movements in reading: Perceptual and language processes (pp. 141-150). New York: Academic Press.

Westheimer, G. (1993). Phosphor persistence in oscilloscopic displays. Vision Research, 33, 2337-2338.

White, C. W. (1976). Visual masking during pursuit eye movements. Journal of Experimental Psychology: Human Perception and Performance, 2, 469-478.

Whittaker, S. G., Cummings, R. W., \& Swieson, L. R. (1991). Saccade control without a fovea. Vision Research, 31, 2209-2218.

Wilkins, A. J. (1986). Intermittent illumination from fluorescent lighting and visual display units affects movements of the eyes across text. Human Factors, 28, 75-81.

Williams, D. E., Reingold, E., Moscovitch, M., \& Behrmann, M. (1997). Patterns of eye movements during parallel and serial visual search tasks. Canadian Journal of Experimental Psychology, 51, 151-164.

Wolf, W., Hauske, G., \& Lupp, U. (1978). How presaccadic gratings modify post-saccadic modulation transfer function. Vision Research, $18,1173-1179$.

Wolf, W., Hauske, G., \& Lupp, U. (1980). Interaction of pre- and post-saccadic patterns having the same coordinates in space. Vision Research, 20, 117-125.

Wolverton, G. S., \& Zola, D. (1983). The temporal characteristics of visual information extraction during reading. In K. Rayner (Ed.), Eye movements in reading: Perceptual and language processes (pp. 4152). New York: Academic Press.

Yarbus, A. (1967). Eye movements and vision. New York: Plenum Press. Ygge, J., \& Jacobson, C. (1994). Asymmetrical saccades in reading. In J. Ygge \& G. Lennerstrand (Eds.), Eye movements in reading (pp. 301-313). Oxford, England: Pergamon Press.

Zagar, D., Pynte, J., \& Rativeau, S. (1997). Evidence for early-closure attachment on first-pass reading times in French. Quarterly Journal of Experimental Psychology, 50A, 421-438.

Zangemeister, W. H., Sherman, K., \& Stark, L. (1995). Evidence for a global scanpath strategy in viewing abstract compared with realistic images. Neuropsychologia, 33, 1009-1025.

Zangwill, O. L., \& Blakemore, C. (1972). Dyslexia: Reversal of eye movements during reading. Neuropsychologia, 10, 371-373.

Zelinsky, G. J. (1996). Using eye saccades to assess the selectivity of search movements. Vision Research, 36, 2177-2187.

Zelinsky, G. J., Rao, R. P. N., Hayhoe, M. M., \& Ballard, D. H. (1997). Eye movements reveal the spatiotemporal dynamics of visual search. Psychological Science, 8, 448-453.

Zelinsky, G. J., \& Sheinberg, D. L. (1997). Eye movements during parallel-serial visual search. Journal of Experimental Psychology: Human Perception and Performance, 23, 244-262.

Zihl, J. (1995). Eye movement patterns in hemianopic dyslexia. Brain, $118,891-912$.

Zingale, C. M., \& Kowler, E. (1987). Planning sequences of saccades. Vision Research, 27, 1327-1341.

Zola, D. (1984). Redundancy and word perception during reading. Perception \& Psychophysics, 36, 277-284.

Received August 18, 1997

Revision received March 6, 1998

Accepted March 20, 1998 